TRANSACTIONS OF THE

AMERICAN MATHEMATICAL SOCIETY

Volume 355, Number 6, Pages 2341-2384

S 0002-9947(03)03266-5

Article electronically published on February 4, 2003

\title{
INDUCTION THEOREMS OF SURGERY OBSTRUCTION GROUPS
}

\author{
MASAHARU MORIMOTO \\ Dedicated to Professor Anthony Bak for his sixtieth birthday
}

\begin{abstract}
Let $G$ be a finite group. It is well known that a Mackey functor $\{H \mapsto M(H)\}$ is a module over the Burnside ring functor $\{H \mapsto \Omega(H)\}$, where $H$ ranges over the set of all subgroups of $G$. For a fixed homomorphism $w$ : $G \rightarrow\{-1,1\}$, the Wall group functor $\left\{H \mapsto L_{n}^{h}\left(\mathbb{Z}[H],\left.w\right|_{H}\right)\right\}$ is not a Mackey functor if $w$ is nontrivial. In this paper, we show that the Wall group functor is a module over the Burnside ring functor as well as over the Grothendieck-Witt ring functor $\left\{H \mapsto \mathrm{GW}_{0}(\mathbb{Z}, H)\right\}$. In fact, we prove a more general result, that the functor assigning the equivariant surgery obstruction group on manifolds with middle-dimensional singular sets to each subgroup of $G$ is a module over the Burnside ring functor as well as over the special Grothendieck-Witt ring functor. As an application, we obtain a computable property of the functor described with an element in the Burnside ring.
\end{abstract}

\section{INTRODUCTION}

Dress' induction theory ([10], 11], [12]) of Mackey functors has been useful for algebraic computation of Wall's surgery obstruction groups (27]) with trivial orientation homomorphisms and related groups (cf. [6, [13, [14]) as well as for applications in transformation groups (e.g. [16], [18], [25], [26]). In this paper, we develop induction theory for surgery obstruction groups appearing in [4, [5] and [19, which allows nontrivial orientation homomorphisms, and by using this generalization and [22, Theorem 1.1] we can construct various group actions on smooth manifolds (e.g. 4], [15, 16, [17, 20], 21, 24]).

Throughout this paper, let $G$ be a finite group, $\mathcal{S}(G)$ the set of all subgroups of $G$, and $R$ a principal ideal domain (possibly a commutative field). Hence $R$ is a commutative ring and any finitely generated projective $R$-module is free over $R$. An $R$-module is always assumed to be finitely generated over $R$, unless otherwise stated.

Let $\mathrm{GW}_{0}(R, G)$ denote the Grothendieck-Witt ring in A. Dress 11]. It is well known that the functor $H \mapsto \mathrm{GW}_{0}(R, H), H \in \mathcal{S}(G)$, with canonical correspondence of morphisms is a Green functor, which is a special case of Theorem 11.3 since $\mathrm{GW}_{0}(R, G)=\mathrm{GW}_{0}(R, G, \emptyset)$. Let $\mathcal{C}(G)$ denote the set of all cyclic subgroups

Received by the editors January 1, 2002.

2000 Mathematics Subject Classification. Primary 19G12, 19G24, 19J25; Secondary 57R67.

Key words and phrases. Induction, restriction, Burnside ring, Grothendieck group, Witt group, equivariant surgery.

Partially supported by a Grant-in-Aid for Scientific Research (Kakenhi).

(C)2003 American Mathematical Society 
of $G$. By [11, Theorem 1], the functor $H \mapsto \mathrm{GW}_{0}(R, H)$ is $\mathcal{C}(G)$-hypercomputable in the sense of A. Bak [2]. Let $w: G \rightarrow\{-1,1\}$ be a homomorphism and $n=2 k$ an even integer. If $w$ is nontrivial, the Wall group functor $H \mapsto L_{n}^{h}\left(R[H],\left.w\right|_{H}\right)$ ([27]), $H \in \mathcal{S}(G)$, is not a Mackey functor. Since $L_{n}^{h}(R[G], w)=\mathrm{WQ}_{0}(\boldsymbol{A}, \emptyset)$ with $A=\left(R, G, \emptyset, \emptyset,(-1)^{k}, w\right)$, Propositions 12.7 and 2.6 imply that the Wall group functor is a $w$-Mackey functor in the sense of Definition 2.2 and a module over the Burnside ring functor. Furthermore, the Wall group functor is a module over the functor $H \mapsto \mathrm{GW}_{0}(R, H)$, which is a special case of Theorem 12.10. Thus, we obtain the theorem:

Theorem 1.1. Let $w: G \rightarrow\{-1,1\}$ be a homomorphism and $n$ an even integer. Then the Wall group functor $H \mapsto L_{n}^{h}\left(R[H],\left.w\right|_{H}\right), H \in \mathcal{S}(G)$, is $\mathcal{C}(G)$ hypercomputable.

The main purpose of this paper is to study the induction-restriction theory of the equivariant surgery obstruction group $\operatorname{SWQ}_{0}\left(R, G, Q, S, \Theta_{G}\right)$ obtained by Bak and Morimoto [5, which consists of equivalence classes of special $\lambda$-quadratic $R[G]$ modules. This surgery obstruction group is determined by a datum

$$
\mathcal{D}=\left(R, G, Q, S, \lambda, w, \Theta_{G}, \rho^{(2)}\right) .
$$

The ingredient $\lambda$ stands for a symmetry, namely either 1 or -1 . Let $G(2)$ denote the subset of $G$ consisting of all elements of order 2. An element $g \in G(2)$ is called $\lambda$-symmetric or $\lambda$-quadratic if $g=\lambda w(g) g^{-1}$ or $g=-\lambda w(g) g^{-1}$, respectively. The ingredients $Q$ and $S$ are conjugation-invariant subsets of $G(2)$ consisting of $\lambda$-quadratic elements and $\lambda$-symmetric ones, respectively. Let $\mathfrak{P}(S)$ denote the set of all subsets of $S$. In a general case, $\Theta_{G}$ stands for a finite $G$-set and $\rho^{(2)}$ is a $G$-map $\Theta_{G} \rightarrow \mathfrak{P}(S)$. In the case where $S$ and $\Theta_{G}$ are both empty and $\lambda=(-1)^{k}$, the group $\mathrm{SWQ}_{0}\left(\mathbb{Z}, G, Q, S, \Theta_{G}\right)$ coincides with the Bak group $W_{2 k}(\mathbb{Z}[G], \Gamma Q, w)$ (see [19]); if moreover $Q$ is also empty, then the group is nothing but the Wall group $L_{2 k}^{h}(\mathbb{Z}[G], w)$ (see [27]).

In the current section, since the case $\Theta_{G}=S$ has interesting applications (e.g. [4], [15], [16]), we let $\Theta_{G}$ and $\rho^{(2)}$ be the same as the set $S$ and the map $s \mapsto\{s\}$, $s \in S$, respectively.

We detail the pairing

$$
\mathrm{SGW}_{0}(\mathbb{Z}, G, S, S) \times \mathrm{SWQ}_{0}(\mathbb{Z}, G, Q, S, S) \longrightarrow \mathrm{SWQ}_{0}(\mathbb{Z}, G, Q, S, S)
$$

in Sections 9 and 10 and show that $\mathrm{SWQ}_{0}(\mathbb{Z}, G, Q, S, S)$ is a module over the special Grothendieck-Witt ring $\mathrm{SGW}_{0}(\mathbb{Z}, G, S, S)$, which corrects the invalid description [15, page 513 , lines $9-10]$ of the pairing.

The groups $\mathrm{GW}_{0}(R, G)$ and $L_{n}^{h}(R[G], w)$ with $n=2 k$ have the hyperelementary computability. Dress proved this fact by studying the index of the subgroup $I\left(\mathfrak{H}_{\Sigma}(G), \mathrm{GW}_{0}\right)$ of $\mathrm{GW}_{0}(R, G)$ ([11, Theorem 1]), which we call the Dress index. The theorem looks technical but is fundamental. It is natural to regard the Burnside ring as a generalization of the ring of integers in the theory of transformation groups. Thus, one expects that some computability of the groups $\mathrm{SGW}_{0}(\mathbb{Z}, G, S, S)$ and $\mathrm{SWQ}_{0}(\mathbb{Z}, G, Q, S, S)$ can be described with an element in the Burnside ring instead of the Dress index. The following theorems are obtained in this respect.

Let $1_{\Omega(G)}$ denote the unit of the Burnside ring $\Omega(G)$. 
Theorem 1.2. Let $S$ be a conjugation-invariant subset of $G$ consisting of elements of order 2 , let $\mathcal{F}$ be a conjugation-invariant set of subgroups of $G$ such that

$$
S \times S \subset \bigcup_{H \in \mathcal{F}} H \times H
$$

and let $\beta$ be an element of the Burnside ring $\Omega(G)$ such that

$$
\operatorname{Res}_{H}^{G} \beta=1_{\Omega(H)} \quad \text { for any } H \in \mathcal{F} .
$$

If $\mathcal{F}$ contains all 2-hyperelementary (resp. cyclic) subgroups of $G$, then, for an arbitrary element $x \in \mathrm{SGW}_{0}(R, G, S, S)$,

$$
\left(1_{\Omega(G)}-\beta\right)^{2} x=0
$$

(resp. $\left(1_{\Omega(G)}-\beta\right)^{2 k+3} x=0$, where $|G|=2^{k} m$ with $m$ odd).

We say that $R$ is square identical if

$$
r^{2} \equiv r \quad \bmod 2 R \quad \text { for all } r \in R .
$$

Theorem 1.3. Let $S, \beta$ and $\mathcal{F}$ be as in the theorem above. Suppose that $R$ is square identical, and each element of $S$ is $\lambda$-symmetric. Let $Q$ be a conjugationinvariant subset of $G$ consisting of $\lambda$-quadratic elements of order 2 . If $\mathcal{F}$ contains all 2-hyperelementary (resp. cyclic) subgroups of $G$, then for an arbitrary element $x$ of $\mathrm{SWQ}_{0}(R, G, Q, S, S)$,

$$
\left(1_{\Omega(G)}-\beta\right)^{2} x=0
$$

(resp. $\left(1_{\Omega(G)}-\beta\right)^{2 k+3} x=0$, where $|G|=2^{k}$ m with $m$ odd).

Note that the datum $\mathcal{D}=\left(R, G, Q, S, \lambda, w, S, \rho^{(2)}\right)$, where $\rho^{(2)}: S \rightarrow \mathfrak{P}(S)$ is the "identity map" $s \mapsto\{s\}$, yields the datum $\mathcal{D}=\left(R, H, Q \cap H, S \cap H, \lambda,\left.w\right|_{H}, S \cap\right.$ $\left.H,\left.\rho^{(2)}\right|_{S \cap H}\right)$ and determines the group $\operatorname{SWQ}_{0}(R, H, Q \cap H, S \cap H, S \cap H)$ for each subgroup $H$ of $G$.

Theorem 1.4. Let $G$ be a nonsolvable group and let $R, Q$ and $S$ be as in the previous theorem. Then

$$
\operatorname{SWQ}_{0}(R, G, Q, S, S)=\sum_{H} \operatorname{Ind}_{H}^{G} \operatorname{SWQ}_{0}(R, H, Q \cap H, S \cap H, S \cap H),
$$

and the restriction homomorphism

$$
\text { Res : } \operatorname{SWQ}_{0}(R, G, Q, S, S) \longrightarrow \bigoplus_{H} \operatorname{SWQ}_{0}(R, H, Q \cap H, S \cap H, S \cap H)
$$

is injective, where $H$ ranges over the set of all solvable subgroups of $G$.

Each of Theorems 1.2 1.4 is slightly generalized in Section 13 .

The organization of the paper is as follows. In Section 2 we define a $w$-Mackey functor, a Green functor, and a module over a Green functor. In Section 3, we observe basic properties of $\Theta$-positioned $R[G]$-modules, namely induction-restriction properties and the Mackey double coset formula. Section 4 is devoted to observing induction-restriction properties of $\Theta$-positioned Hermitian $R[G]$-modules as well as defining their Grothendieck-Witt rings. In Section 5 , we introduce the $\nabla$-invariant of $\Theta$-positioned Hermitian $R[G]$-modules and define the special Grothendieck-Witt groups. Similarly to Wall's surgery theory, $R[G]$-valued $\lambda$-Hermitian forms are indispensable objects to equivariant surgery theory on manifolds with middledimensional singular sets. Section 6 is devoted to observing induction-restriction 
properties of $R[G]$-valued $\lambda$-Hermitian modules. Sections 7 and 8 are devoted to defining the Witt groups and the special Witt groups of $\Theta$-positioned quadratic $R[G]$-modules, respectively. The tensor product of a Hermitian $R[G]$-module and a quadratic $R[G]$-module is introduced in Section 9 and it is discussed with $\nabla$ invariants in Section 10 Section 11 is devoted to showing that the GrothendieckWitt rings and special Grothendieck-Witt rings are Green functors (possibly without unit). In Section 12 we show that the bifunctor assigning the $H$-surgery obstruction group to a subgroup $H$ of $G$ is a module over the special Grothendieck-Witt ring functor. In Section 13, we present applications relevant to $G$-surgery.

Acknowledgements. I wish to express my heartfelt gratitude to Anthony Bak for his numerous valuable suggestions to my research related to $K$-theory. If it had not been for his influence, this work would not have been done. In addition, I thank the referee for his comments on elaborating upon the manuscript.

\section{Bifunctors, $w$-Mackey functors and Green functors}

Let $\mathcal{G}$ denote the category whose objects are subgroups of $G$ and whose morphisms are inclusions $j_{H, K}: H \rightarrow K$, where $H \subset K \subset G$, conjugations $c_{(H, g)}$ : $H \rightarrow g H g^{-1} ; a \mapsto g a g^{-1}$, where $H \subset G$ and $g \in G$, and compositions of those maps. Let $\mathcal{A}$ stand for the category whose objects are abelian groups and whose morphisms are group homomorphisms. We denote by $\mathbb{Z}[\mathcal{S}(G)]$ the free abelian group generated by all elements of $\mathcal{S}(G)$; hence each element of $\mathbb{Z}[\mathcal{S}(G)]$ has the form $\sum_{H} n_{H} H$ with $n_{H} \in \mathbb{Z}$. Let $\Omega(G)$ denote the Burnside ring of $G$ (cf. [7], [8], 9], 23]). In fact, $\Omega(G)$ is the free abelian group generated by all $G$-isomorphism classes $[G / H]$ of finite $G$-sets $G / H$ with $H \in \mathcal{S}(G)$. Clearly, one has the canonical homomorphism from $\mathbb{Z}[\mathcal{S}(G)]$ to $\Omega(G)$ such that $H \mapsto[G / H]$. In this paper, we mean by a bifunctor

$$
L=\left(L^{*}, L_{*}\right): \mathcal{G}(G) \rightarrow \mathcal{A}
$$

a pair consisting of a contravariant functor $L^{*}: \mathcal{G}(G) \rightarrow \mathcal{A}$ and a covariant functor $L_{*}: \mathcal{G}(G) \rightarrow \mathcal{A}$ such that $L_{*}(H)=L^{*}(H)$, which is written as $L(H)$, for all $H \in \mathcal{S}(G)$. If the context is clear, $f^{*}$ and $f_{*}$ stand for $L^{*}(f)$ and $L_{*}(f)$ respectively, and $\operatorname{Res}_{H}^{K}$ and $\operatorname{Ind}_{H}^{K}$ stand for $L^{*}\left(j_{H, K}\right)$ and $L_{*}\left(j_{H, K}\right)$ respectively. Each bifunctor $L=\left(L^{*}, L_{*}\right): \mathcal{G} \rightarrow \mathcal{A}$ possesses the canonical pairing

$$
\mathbb{Z}[\mathcal{S}(G)] \times L(G) \longrightarrow L(G) ;\left(\sum_{H} n_{H} H, x\right) \longmapsto \sum_{H} n_{H} \operatorname{Ind}_{H}^{G}\left(\operatorname{Res}_{H}^{G} x\right),
$$

for $n_{H} \in \mathbb{Z}$ and $x \in L(G)$. It is interesting to look for a sufficient condition so that the pairing (2.1) factors through a pairing

$$
\Omega(G) \times L(G) \longrightarrow L(G) .
$$

If $L$ is a Mackey functor, then, as was seen in [7, Proposition 6.2.3], the pairing (2.1) factors through a pairing (2.2). In the case where the orientation homomorphism $w: G \rightarrow\{-1,1\}$ is not trivial, the Wall group functor $H \mapsto L_{n}^{h}\left(\mathbb{Z}[H],\left.w\right|_{H}\right)$, $H \in \mathcal{S}(G)$, is not a Mackey functor; however, it will turn out that the associated pairing (2.1) factors through (2.2).

Let $L: \mathcal{G} \rightarrow \mathcal{A}$ be a bifunctor. Note that the kernel of the canonical map $\mathbb{Z}[\mathcal{S}(G)] \rightarrow \Omega(G)$ is

$$
\left\langle H-g H g^{-1} \mid H \in \mathcal{S}(G), g \in G\right\rangle_{\mathbb{Z}}
$$


If

$$
L_{*}\left(j_{H, G}\right) L^{*}\left(j_{H, G}\right)=L_{*}\left(j_{g H g^{-1}, G}\right) L^{*}\left(j_{g H g^{-1}, G}\right) \quad(\forall H \in \mathcal{S}(G), \forall g \in G),
$$

then the pairing (2.1) factors through (2.2).

Proposition 2.1. Suppose $L_{*}\left(c_{\left(g H g^{-1}, g^{-1}\right)}\right)=L^{*}\left(c_{(H, g)}\right)$ for all $H \in \mathcal{S}(G)$ and $g \in G$. Then the equality (2.3) holds if and only if

$$
L^{*}\left(c_{(G, g)}\right) L_{*}\left(j_{H, G}\right) L^{*}\left(j_{H, G}\right)=L_{*}\left(j_{H, G}\right) L^{*}\left(j_{H, G}\right) L^{*}\left(c_{(G, g)}\right)
$$

for all $H \in \mathcal{S}(G)$ and $g \in G$.

Proof. By definition, the diagrams

$$
\begin{array}{cc}
L(G) \stackrel{L^{*}\left(j_{H, G}\right)}{\longrightarrow} L(H) \\
L(G) \frac{L^{*}\left(c_{\left(G, g^{-1}\right)}\right)}{\longrightarrow} \stackrel{L^{*}\left(c_{\left(g g^{-1}, g^{-1}\right)}\right)}{\longrightarrow} L\left(g H g^{-1}\right)
\end{array}
$$

and

$$
\begin{array}{cc}
L(H) \stackrel{L_{*}\left(j_{H, G}\right)}{\longrightarrow} L(G) \\
L_{*}\left(c_{\left(g g^{-1}, g^{-1}\right)}\right) \uparrow & \uparrow_{L_{*}\left(c_{\left(G, g^{-1}\right)}\right)}^{\longrightarrow} L\left(g H g^{-1}\right) \underset{L_{*}\left(j_{g H g^{-1}, G}\right)}{\longrightarrow} L(G)
\end{array}
$$

commute. By using the hypothesis above, we obtain the commutative diagram

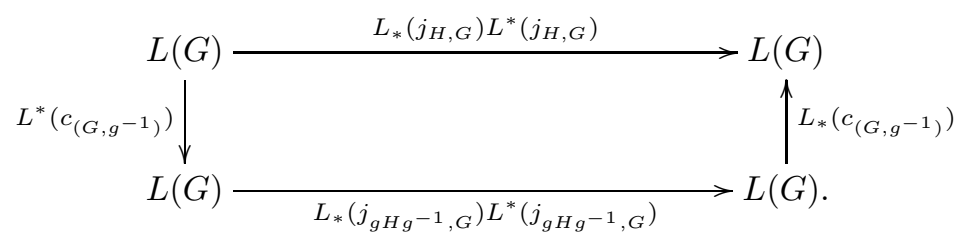

Thus (2.3) holds if and only if

$$
L_{*}\left(j_{g H g^{-1}, G}\right) L^{*}\left(j_{g H g^{-1}, G}\right)=L_{*}\left(c_{\left(G, g^{-1}\right)}\right) L_{*}\left(j_{g H g^{-1}, G}\right) L^{*}\left(j_{g H g^{-1}, G}\right) L^{*}\left(c_{\left(G, g^{-1}\right)}\right),
$$

namely

$$
L^{*}\left(c_{\left(G, g^{-1}\right)}\right) L_{*}\left(j_{g H g^{-1}, G}\right) L^{*}\left(j_{g H g^{-1}, G}\right)=L_{*}\left(j_{g H g^{-1}, G}\right) L^{*}\left(j_{g H g^{-1}, G}\right) L^{*}\left(c_{\left(G, g^{-1}\right)}\right) .
$$

This concludes the proposition.

Let $w: G \rightarrow\{-1,1\}$ be a homomorphism. We introduce a slight generalization of a Mackey functor (cf. [2], [7]).

Definition 2.2. A bifunctor $M=\left(M^{*}, M_{*}\right)$ from $\mathcal{G}$ to $\mathcal{A}$ is called a $w$-Mackey functor if the following conditions (1)-(3) are fulfilled:

(1) $M_{*}\left(c_{(H, g)}\right)=M^{*}\left(c_{\left(g H g^{-1}, g^{-1}\right)}\right)$ for all $H \in \mathcal{S}(G)$ and $g \in G$,

(2) $M^{*}\left(c_{(H, h)}\right)=w(h) i d_{M(H)}$ (hence $\left.M_{*}\left(c_{(H, h)}\right)=w(h) i d_{M(H)}\right)$ for all $H \in$ $\mathcal{S}(G)$ and $h \in H$,

(3) $M^{*}\left(j_{K, G}\right) \circ M_{*}\left(j_{H, G}\right)$ coincides with

$$
\bigoplus_{K g H \in K \backslash G / H} M_{*}\left(j_{K \cap g H g^{-1}, K}\right) \circ\left(w(g) M_{*}\left(c_{\left(H \cap g^{-1} K g, g\right)}\right)\right) \circ M^{*}\left(j_{H \cap g^{-1} K g, H}\right)
$$


for any $H, K \in \mathcal{S}(G)$.

A $w$-Mackey functor for trivial $w$ is an ordinary Mackey functor. We will see that if $w$ is nontrivial, then the Wall group functor $H \mapsto L_{n}^{h}\left(\mathbb{Z}[H],\left.w\right|_{H}\right)$ is not an ordinary Mackey functor but a $w$-Mackey functor (cf. Propositions 6.6, 6.7 6.8 12.4. 12.5 and 12.6. The next proposition is clear by definition.

Proposition 2.3. If $M=\left(M^{*}, M_{*}\right)$ is a w-Mackey functor, then $L=\left(L^{*}, L_{*}\right)$, given so that $L(H)=M(H), L^{*}\left(j_{H, K}\right)=M^{*}\left(j_{H, K}\right), L_{*}\left(j_{H, K}\right)=M_{*}\left(j_{H, K}\right)$, $L^{*}(c(H, g))=w(g) M^{*}(c(H, g))$ and $L_{*}(c(H, g))=w(g) M_{*}(c(H, g))$ for all $H \subset K$ and $g \in G$, is a Mackey functor.

In the case above, we say that $L$ is the Mackey functor associated with $M$.

We use the term "Frobenius pairing" in a sense slightly more general than [7], where relevant bifunctors were assumed to be Mackey functors.

Definition 2.4. Let $L, M$ and $N$ be bifunctors from $\mathcal{G}$ to $\mathcal{A}$. A pairing $L \times M \rightarrow N$ is a family of biadditive maps

$$
L(H) \times M(H) \longrightarrow N(H) ;(x, y) \longmapsto x \cdot y,
$$

where $H$ runs over $\mathcal{S}(G)$. Such a pairing is called a Frobenius pairing if the following conditions (1)-(3) are satisfied for any morphism $f: H \rightarrow K$ in $\mathcal{G}$ :

(1) $N^{*}(f)(x \cdot y)=\left(L^{*}(f) x\right) \cdot\left(M^{*}(f) y\right)$ for all $x \in L(K), y \in M(K)$,

(2) $x \cdot M_{*}(f)(y)=N_{*}(f)\left(L^{*}(f)(x) \cdot y\right)$ for all $x \in L(K), y \in M(H)$,

(3) $L_{*}(f)(x) \cdot y=N_{*}(f)\left(x \cdot M^{*}(f)(y)\right)$ for all $x \in L(H), y \in M(K)$.

Each of (2), (3) is referred to as the Frobenius reciprocity law.

Let us recall the definition of a Green functor.

Definition 2.5. A Mackey functor $M=\left(M_{*}, M^{*}\right): \mathcal{G} \rightarrow \mathcal{A}$ is called a Green functor if each $M(H), H \in \mathcal{S}(G)$, is a ring with unit and the associated pairing $M \times M \rightarrow M$ is a Frobenius pairing. If the existence of the unit in $M(H)$ is not guaranteed, then $M$ is referred as a Green functor, possibly without unit.

The Burnside ring functor $H \mapsto \Omega(G)$ is a Green functor. Let $U: \mathcal{G} \rightarrow \mathcal{A}$ be a Green functor. We mean by a $U$-module $L$ (or a module $L$ over $U$ ) a bifunctor $L: \mathcal{G} \rightarrow \mathcal{A}$ equipped with a Frobenius pairing $U \times L \rightarrow L$.

Proposition 2.6. A w-Mackey functor $M$ is a module over the Burnside ring functor.

Proof. Let $L$ be the Mackey functor associated with $M$ in Proposition 2.3. By [7] Proposition 6.2.3], $L$ is a module over the Burnside ring functor. Hence, $L$ satisfies the equality (1) in Proposition 2.1. By using the relations between $M$ and $L$ in Proposition 2.3, we can check that $M$ satisfies the equality (1) in Proposition 2.1 and furthermore that $M$ is a module over the Burnside ring functor.

Proposition 2.7. A module over a Green functor is a module over the Burnside ring functor.

Proof. Let $L=\left(L^{*}, L_{*}\right): \mathcal{G} \rightarrow \mathcal{A}$ be a module over a Green functor $U=\left(U^{*}, U_{*}\right)$ : $\mathcal{G} \rightarrow \mathcal{A}$. Then the associated pairing

$$
\Omega(H) \times L(H) \longrightarrow L(H)
$$


can be defined so that $a \cdot x=\left(a \cdot 1_{U(H)}\right) \cdot x$ for $a \in \Omega(H)$ and $x \in L(H)$, where $1_{U(H)}$ is the identity element of $U(H)$. It is straightforward to check the Frobenius reciprocity laws of the pairing.

\section{3. $\Theta$-Positioned $R[G]$-Modules}

Let $\Theta$ be a finite $G$-set. A pair $(M, \alpha)$ consisting of an $R[G]$-module $M$ and a $G$-map $\alpha: \Theta \rightarrow M$ is called a $\Theta$-positioned $R[G]$-module. Let $H$ and $K$ be finite groups and $\varphi: H \rightarrow K$ a homomorphism. For a finite $H$-set $X$, we define the $K$-set $K \times_{H, \varphi} X$ as the quotient set of $K \times X$ with respect to the equivalence relation $\sim$ generated by $(k \varphi(h), x) \sim(k, h x), h \in H$. The set $K \times_{H, \varphi} X$ is also denoted by $K \times_{\varphi} X$ or $K \times_{H} X$ if the context is clear. For an $R[H]$-module $M$, the $R[K]$-module $R[K] \otimes_{R[H], \varphi} M$ is defined as follows. Let $F(R[K] \times M)$ denote the $R$-free module with basis $R[K] \times M$ which may not be finitely generated over $R$.

Let $T$ denote the $R$-submodule generated by all elements of the form

$$
\begin{aligned}
& r(a, x)-(r a, x), \quad r(a, x)-(a, r x), \\
& (a+b, x)-(a, x)-(b, x), \quad(a, x+y)-(a, x)-(a, y), \text { or } \\
& (a \varphi(h), x)-(a, h x),
\end{aligned}
$$

where $r$ ranges over $R, a$ and $b$ over $R[K], x$ and $y$ over $M$, and $h$ over $H$. Then $R[K] \otimes_{R[H], \varphi} M$ is defined to be the quotient module $F(R[K] \times M) / T$, which will also be denoted by $R[K] \otimes_{\varphi} M$ or $R[K] \otimes_{R[H]} M$. The element of the module represented by $(a, x) \in F(R[K] \times M)$ is denoted by $a \otimes_{R[H], \varphi} x$, which will also be written as $a \otimes_{\varphi} x, a \otimes_{R[H]} x$ or $a \otimes x$ if the context is clear. The $K$-action on $R[K] \otimes_{R[H], \varphi} M$ is given by $\left(k, a \otimes_{R[H], \varphi} x\right) \mapsto(k a) \otimes_{R[H], \varphi} x$.

Let $\Theta_{H}$ be a finite $H$-set, $\Theta_{K}$ a finite $K$-set, and $\psi: \Theta_{H} \rightarrow \Theta_{K}$ a $\varphi$-equivariant map, namely

$$
\psi(h t)=\varphi(h) \psi(t) \quad\left(h \in H, t \in \Theta_{H}\right) .
$$

Let $\varphi$ stand for the pair $(\varphi, \psi)$.

For a $\Theta_{K}$-positioned $R[K]$-module $N=(N, \beta)$, we define the $\Theta_{H}$-positioned $R[H]$-module $\boldsymbol{\varphi}^{\#} \boldsymbol{N}=\left(\varphi^{\#} N, \psi^{\#} \beta\right)$ so that the underlying $R$-module of $\varphi^{\#} N$ is the same as $N$ but the $H$-action on $\varphi^{\#} N$ is given by $(h, x) \mapsto \varphi(h) x$ for $h \in H$, $x \in \varphi^{\#} N$, and $\psi^{\#} \beta: \Theta_{H} \rightarrow \varphi^{\#} N$ is given by $\psi^{\#} \beta(t)=\beta(\psi(t))$ for $t \in \Theta_{H}$.

Proposition 3.1. Let $\varphi: H \rightarrow K$ and $\psi: \Theta_{H} \rightarrow \Theta_{K}$ be as above and let $\boldsymbol{N}_{i}=$ $\left(N_{i}, \beta_{i}\right), i=1$, 2, be $\Theta_{K}$-positioned $R[K]$-modules. Then $\boldsymbol{\varphi}^{\#} \boldsymbol{N}_{1} \otimes_{R} \boldsymbol{\varphi}^{\#} \boldsymbol{N}_{2}=$ $\varphi^{\#}\left(N_{1} \otimes_{R} N_{2}\right)$; namely, $\left(\varphi^{\#} N_{1} \otimes_{R} \varphi^{\#} N_{2}, \psi^{\#} \beta_{1} \otimes_{R} \psi^{\#} \beta_{2}\right)$ is canonically isomorphic to $\left(\varphi^{\#}\left(N_{1} \otimes_{R} N_{2}\right), \psi^{\#}\left(\beta_{1} \otimes_{R} \beta_{2}\right)\right)$.

Proof. By definition, the underlying $R$-modules of $\varphi^{\#} N_{1} \otimes_{R} \varphi^{\#} N_{2}$ and $\varphi^{\#}\left(N_{1} \otimes_{R}\right.$ $N_{2}$ ) are $N_{1} \otimes_{R} N_{2}$. One can check without difficulties that the $K$-actions of the two modules coincide. Moreover, we have

$$
\left(\psi^{\#} \beta_{1} \otimes_{R} \psi^{\#} \beta_{2}\right)(t)=\beta_{1}(\psi(t)) \otimes_{R} \beta_{2}(\psi(t))=\psi^{\#}\left(\beta_{1} \otimes_{R} \beta_{2}\right)(t)
$$

for all $t \in \Theta_{H}$.

To the contrary, for a $\Theta_{H}$-positioned $R[H]$-module $M=(M, \alpha)$, we define the $\Theta_{K}$-positioned $R[K]$-module $\boldsymbol{\varphi}_{\#} \boldsymbol{M}=\left(\varphi_{\#} M, \psi_{\#} \alpha\right)$ by $\varphi_{\#} M=R[K] \otimes_{R[H], \varphi} M$ and

$\psi_{\#} \alpha(t)=\sum_{\left[k, t^{\prime}\right]}\left\{k \otimes_{\varphi} \alpha\left(t^{\prime}\right) \mid\left[k, t^{\prime}\right] \in K \times_{H, \varphi} \Theta_{H}\right.$ such that $\left.k \psi\left(t^{\prime}\right)=t\right\} \quad$ for $t \in \Theta_{K}$. 
The $K$-equivariance of the map $\psi_{\#} \alpha$ holds because, for $a \in K$ and $t \in \Theta_{K}$,

$$
\begin{aligned}
\psi_{\#} \alpha(a t) & =\sum_{\left[k, t^{\prime}\right] \in K \times_{H, \varphi} \Theta_{H}}\left\{k \otimes_{\varphi} \alpha\left(t^{\prime}\right) \mid k \psi\left(t^{\prime}\right)=a t\right\} \\
& =\sum_{\left[k, t^{\prime}\right] \in K \times_{H, \varphi} \Theta_{H}}\left\{k \otimes_{\varphi} \alpha\left(t^{\prime}\right) \mid a^{-1} k \psi\left(t^{\prime}\right)=t\right\} \\
& =\sum_{\left[a k^{\prime}, t^{\prime}\right] \in K \times_{H, \varphi} \Theta_{H}}\left\{a k^{\prime} \otimes_{\varphi} \alpha\left(t^{\prime}\right) \mid k^{\prime} \psi\left(t^{\prime}\right)=t\right\} \\
& =a \sum_{\left[a k^{\prime}, t^{\prime}\right] \in K \times_{H, \varphi} \Theta_{H}}\left\{k^{\prime} \otimes_{\varphi} \alpha\left(t^{\prime}\right) \mid k^{\prime} \psi\left(t^{\prime}\right)=t\right\} \\
& =a \sum_{\left[k^{\prime}, t^{\prime}\right] \in K \times_{H, \varphi} \Theta_{H}}\left\{k^{\prime} \otimes_{\varphi} \alpha\left(t^{\prime}\right) \mid k^{\prime} \psi\left(t^{\prime}\right)=t\right\} \\
& =a \psi_{\#} \alpha(t) .
\end{aligned}
$$

Proposition 3.2. Let $H$ be a subgroup of $G, \boldsymbol{M}=(M, \alpha)$ a $\Theta_{H}$-positioned $R[H]$ module, $g$ an element of $G$, and $\psi: \Theta_{H} \rightarrow \Theta_{g H g^{-1}}$ a $c_{H, g^{-}}$equivariant bijection. Then the diagram

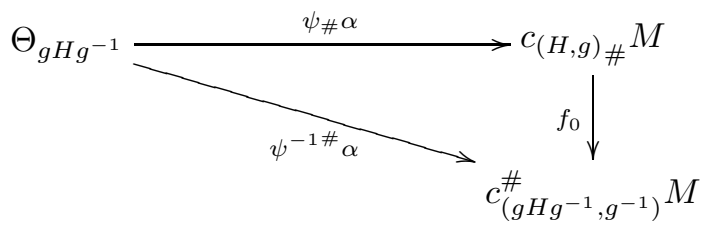

commutes, where $f_{0}: c_{(H, g)} M \rightarrow c_{\left(g H g^{-1}, g^{-1}\right)}^{\#} M$ is the $R\left[g H g^{-1}\right]$-isomorphism such that

$$
f_{0}\left(e \otimes_{H, c_{(H, g)}} x\right)=x \quad \text { for } x \in M .
$$

Proof. Let $t$ be an element of $\Theta_{H}$. Then by definition we have $\psi_{\#} \alpha(\psi(t))=$ $e \otimes_{H, c_{(H, g)}} \alpha(t)$ and $\psi^{-1 \#} \alpha(\psi(t))=\alpha(t)$, which concludes the proposition.

Proposition 3.3. Let $\left(H, \Theta_{H}\right),\left(K, \Theta_{K}\right)$, and $\boldsymbol{\varphi}=(\varphi, \psi)$ be as above. Then for a $\Theta_{H}$-positioned $R[H]$-module $(M, \alpha)$ and a $\Theta_{K}$-positioned $R[K]$-module $(N, \beta)$, the Frobenius reciprocity law holds; namely, the following diagram commutes:

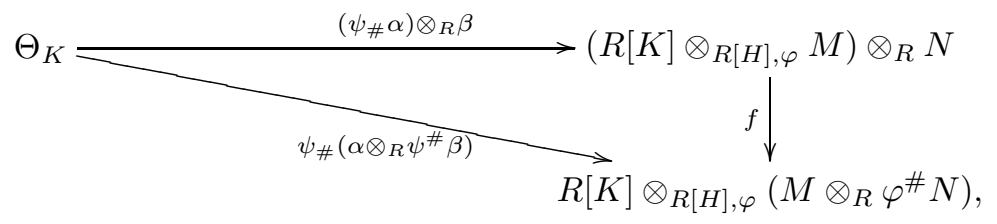

where $f$ is the canonical isomorphism such that $f\left(\left(k \otimes_{\varphi} x\right) \otimes y\right)=k \otimes_{\varphi}\left(x \otimes k^{-1} y\right)$ for $k \in K, x \in M$ and $y \in N$.

The commutability above is referred to as $\left(\psi_{\#} \alpha\right) \otimes_{R} \beta=\psi_{\#}\left(\alpha \otimes_{R} \psi^{\#} \beta\right)$. 
Proof. The proof runs as follows:

$$
\begin{aligned}
\left(\left(\psi_{\#} \alpha\right) \otimes_{R} \beta\right)(t) & =\sum_{\left[k, t^{\prime}\right] \in K \times_{H} \Theta_{H}}\left\{k \otimes_{\varphi} \alpha\left(t^{\prime}\right) \mid k \psi\left(t^{\prime}\right)=t\right\} \otimes \beta(t) \\
& =\sum_{\left[k, t^{\prime}\right] \in K \times_{H} \Theta_{H}}\left\{\left(k \otimes_{\varphi} \alpha\left(t^{\prime}\right)\right) \otimes \beta(t) \mid k \psi\left(t^{\prime}\right)=t\right\} \\
& =\sum_{\left[k, t^{\prime}\right] \in K \times_{H} \Theta_{H}}\left\{\left(k \otimes_{\varphi} \alpha\left(t^{\prime}\right)\right) \otimes k \beta\left(\psi\left(t^{\prime}\right)\right) \mid k \psi\left(t^{\prime}\right)=t\right\} \\
& \stackrel{f}{=} \sum_{\left[k, t^{\prime}\right] \in K \times_{H} \Theta_{H}}\left\{k \otimes_{\varphi}\left(\alpha\left(t^{\prime}\right) \otimes \beta\left(\psi\left(t^{\prime}\right)\right)\right) \mid k \psi\left(t^{\prime}\right)=t\right\} \\
& =\sum_{\left[k, t^{\prime}\right] \in K \times_{H} \Theta_{H}}\left\{k \otimes_{\varphi}\left(\alpha\left(t^{\prime}\right) \otimes\left(\psi^{\#} \beta\right)\left(t^{\prime}\right)\right) \mid k \psi\left(t^{\prime}\right)=t\right\} \\
& =\sum_{\left[k, t^{\prime}\right] \in K \times_{H} \Theta_{H}}\left\{k \otimes_{\varphi}\left(\alpha \otimes \psi^{\#} \beta\right)\left(t^{\prime}\right) \mid k \psi\left(t^{\prime}\right)=t\right\} \\
& =\psi_{\#}\left(\alpha \otimes_{R} \psi^{\#} \beta\right)(t) .
\end{aligned}
$$

Let $H$ be a subgroup of $G$ and $g$ an element of $G$. Let $c_{(H, g)}: H \rightarrow g H g^{-1}$ stand for the conjugation map by $g$, i.e., $c_{(H, g)}(h)=g h g^{-1}$ for $h \in H$. Let $Z$ be a finite $G$-set, $\Theta_{H}$ an $H$-invariant subset of $Z$, and $\Theta_{g H g^{-1}}$ a $g H g^{-1}$-invariant subset of $Z$ such that $g \Theta_{H}=\Theta_{g H g^{-1}}$. Then the left translation by $g$, namely the map $\ell_{(H, g)}: \Theta_{H} \rightarrow \Theta_{g H g^{-1}} ; t \mapsto g t$, is a $c_{(H, g)}$-equivariant bijection. Let $\boldsymbol{c}_{(H, g)}$ denote the pair $\left(c_{(H, g)}, \ell_{(H, g)}\right)$. If the context is clear, then we abuse $c_{(H, g) \#}$ for $\ell_{(H, g) \#}$, and $c_{(H, g)}^{\#}$ for $\ell_{(H, g)}^{\#}$.

In the special case where $g \in H$, the conjugation map $c_{(H, g)}$ is a map from $H$ to itself. Note that the map

$$
f_{1}: c_{(H, g) \#} M \longrightarrow M ; e \otimes_{c_{(H, g)}} x \longmapsto g x
$$

is an $R[H]$-isomorphism. In addition, the map

$$
f_{2}: c_{(H, g)}^{\#} M \longrightarrow M ; x \longmapsto g^{-1} x
$$

is an $R[H]$-isomorphism.

Proposition 3.4. Let $H$ be a subgroup of $G$ and $\Theta_{H}$ a finite $H$-set. Then for any $\Theta_{H}$-positioned $R[H]$-module $(M, \alpha)$ and $g \in H$, the following diagrams commute:

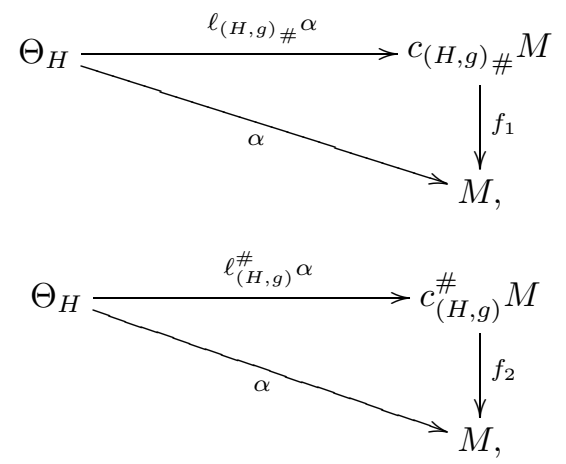


where $f_{1}$ and $f_{2}$ are the $R[H]$-isomorphisms given above.

These commutabilities are referred to as $\ell_{(H, g)} \alpha=\alpha$ (or $\left.c_{(H, g)} \alpha=\alpha\right)$ and $\ell_{(H, g)}^{\#} \alpha=\alpha\left(\right.$ or $\left.c_{(H, g)}^{\#} \alpha=\alpha\right)$, respectively.

Proof. The commutabilities follow from the equalities

$$
\begin{aligned}
f_{1}\left(\ell_{(H, g) \#} \alpha(t)\right) & =\sum_{\left[g h g^{-1}, t^{\prime}\right] \in H \times_{H, c}(H, g)} \Theta_{H}\left\{f_{1}\left(g h g^{-1} \otimes_{c_{(H, g)}} \alpha\left(t^{\prime}\right)\right) \mid g h g^{-1}\left(g t^{\prime}\right)=t\right\} \\
& =\sum_{\left[g h g^{-1}, t^{\prime}\right] \in H \times_{H, c_{(H, g)}} \Theta_{H}}\left\{f_{1}\left(e \otimes_{c_{(H, g)}} \alpha\left(h t^{\prime}\right)\right) \mid g h t^{\prime}=t\right\} \\
& =\sum_{\left[e, t^{\prime \prime}\right] \in H \times_{H, c_{(H, g)}} \Theta_{H}}\left\{f_{1}\left(e \otimes_{c_{(H, g)}} \alpha\left(t^{\prime \prime}\right)\right) \mid g t^{\prime \prime}=t\right\} \\
& =\sum_{\left[e, t^{\prime \prime}\right] \in H \times_{H, c}(H, g)} \Theta_{H}\left\{g \alpha\left(t^{\prime \prime}\right) \mid g t^{\prime \prime}=t\right\} \\
& =\sum_{\left[e, t^{\prime \prime}\right] \in H \times_{H, c}(H, g)}\left\{\alpha(t) \mid g t^{\prime \prime}=t\right\} \\
& =\alpha(t),
\end{aligned}
$$

and

$$
\begin{aligned}
f_{2}\left(\ell_{(H, g)}^{\#} \alpha(t)\right) & =f_{2}\left(\alpha\left(\ell_{(H, g)}(t)\right)\right) \\
& =f_{2}(\alpha(g t)) \\
& =g^{-1} \alpha(g t) \\
& =\alpha(t),
\end{aligned}
$$

for $t \in \Theta_{H}$.

Let $Z$ be a finite $G$-set. Let $\mathcal{S}(G)$ and $\mathfrak{P}(Z)$ denote the set of all subgroups of $G$ and the set of all subsets of $Z$, respectively. We regard $\mathcal{S}(G)$ as a $G$-set by conjugation, and $\mathfrak{P}(Z)$ has the canonical $G$-action. Let $\Theta: \mathcal{S}(G) \rightarrow \mathfrak{P}(G)$; $H \mapsto \Theta_{H}$, be a $G$-map. We say that $\Theta$ is intersection preserving if

$$
\Theta_{H} \cap \Theta_{K}=\Theta_{H \cap K} \quad \text { for all } H, K \in \mathcal{S}(G) \text {. }
$$

Let $H \subset K$ be subgroups of $G$. Then (3.1) implies $\Theta_{H} \subset \Theta_{K}$. Thus, the inclusion map $j_{H, K}: H \rightarrow K$ is automatically associated with the inclusion map $j_{\Theta_{H}, \Theta_{K}}: \Theta_{H} \rightarrow \Theta_{K}$, and hence yields the pair $j_{H, K}=\left(j_{H, K}, j_{\Theta_{H}, \Theta_{K}}\right)$.

Usually, we use $\operatorname{Ind}_{H}^{K}$ for $j_{H, K \#}, j_{\Theta_{H}, \Theta_{K} \#}$ and $j_{H, K \#}$, and $\operatorname{Res}_{H}^{K}$ for $j_{H, K}^{\#}$, $j_{\Theta_{H}, \Theta_{K}}^{\#}$ and $j_{H, K}^{\#}$, if the context is clear.

Next, let $g$ be an element of $G$. Since $\Theta$ is a $G$-map, $\Theta_{g H g^{-1}}=g \Theta_{H}$ holds for any subgroup $H$ of $G$.

Proposition 3.5. Let $\Theta: \mathcal{S}(G) \rightarrow \mathfrak{P}(Z)$ be an intersection-preserving $G$-map.

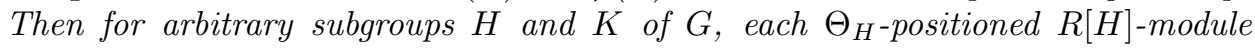
$\boldsymbol{M}=(M, \alpha)$ satisfies the Mackey double coset formula. Namely,

$$
\operatorname{Res}_{K}^{G}\left(\operatorname{Ind}_{H}^{G} \boldsymbol{M}\right)=\bigoplus_{K g H \in K \backslash G / H} \operatorname{Ind}_{K \cap g H g^{-1}}^{K} c_{\left(H \cap g^{-1} K g, g\right)} \operatorname{Res}_{H \cap g^{-1} K g}^{H} \boldsymbol{M} .
$$


More precisely, the following diagram commutes:

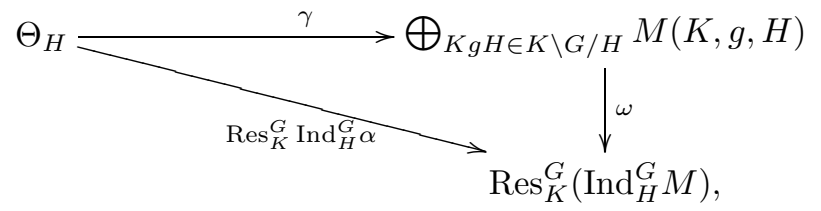

where

$$
\begin{gathered}
M(K, g, H)=\operatorname{Ind}_{K \cap g H g^{-1}}^{K} c_{\left(H \cap g^{-1} K g, g\right) \#} \operatorname{Res}_{H \cap g^{-1} K g}^{H} M \\
=R[K] \otimes_{R\left[K \cap g H g^{-1}\right]}\left(R\left[K \cap g H g^{-1}\right] \otimes_{R\left[H \cap g^{-1} K g\right], c_{\left(H \cap g^{-1} K g, g\right)}} \operatorname{Res}_{H \cap g^{-1} K g}^{H} M\right), \\
\operatorname{Res}_{K}^{G}\left(\operatorname{Ind}_{H}^{G} M\right)=\operatorname{Res}_{K}^{G}\left(R[G] \otimes_{R[H]} M\right), \\
\gamma=\bigoplus_{K g H \in K \backslash G / H} \operatorname{Ind}_{K \cap g H g^{-1}}^{K}\left(\ell_{\left(H \cap g^{-1} K g, g\right)_{\#}}\left(\operatorname{Res}_{H \cap g^{-1} K g}^{H} \alpha\right)\right),
\end{gathered}
$$

and $\omega$ is the $R[K]$-isomorphism such that $\omega\left(k \otimes\left(a \otimes_{c_{\left(H \cap g^{-1} K g, g\right)}} x\right)\right)=k g \otimes\left(g^{-1} a g\right) x \quad$ for $k \in K, a \in K \cap g H^{-1}, x \in M$.

Proof. Let $\alpha: \Theta_{H} \rightarrow M$ be an $H$-map, and let $\left\{g_{1}, \ldots, g_{\ell}\right\}$ be a complete set of representatives of $K \backslash G / H$. For $t \in \Theta_{K}$, we have

$$
\begin{aligned}
& \left(\operatorname{Res}_{K}^{G} \operatorname{Ind}_{H}^{G} \alpha\right)(t) \\
& =\sum\left\{g \otimes \alpha\left(t^{\prime}\right) \mid\left[g, t^{\prime}\right] \in G \times_{H} \Theta_{H}, g t^{\prime}=t\right\} \\
& =\sum_{j=1}^{\ell} \sum\left\{g g_{j} \otimes \alpha\left(t^{\prime}\right) \mid\left[g g_{j}, t^{\prime}\right] \in K g_{j} H \times_{H} \Theta_{H}, g \in K, g g_{j} t^{\prime}=t\right\} \\
& =\sum_{j=1}^{\ell} \sum\left\{g g_{j} \otimes \alpha\left(t^{\prime}\right) \mid\left[g g_{j}, t^{\prime}\right] \in K g_{j} \times_{H \cap g_{j}^{-1} K g_{j}} \Theta_{H}, g \in K, g g_{j} t^{\prime}=t\right\} \\
& =\sum_{j=1}^{\ell} \sum\left\{g g_{j} \otimes \alpha\left(t^{\prime}\right) \mid\left[g g_{j}, t^{\prime}\right] \in K g_{j} \times_{H \cap g_{j}^{-1} K g_{j}} \Theta_{H \cap g_{j}^{-1} K g_{j}},\right. \\
& \left.g \in K, g g_{j} t^{\prime}=t\right\} \quad \text { in } \operatorname{Res}_{K}^{G} \operatorname{Ind}_{H}^{G} M
\end{aligned}
$$

and

$$
\begin{aligned}
& \left.\operatorname{Ind}_{K \cap g_{j} H g_{j}^{-1}}^{K} \ell_{\left(H \cap g_{j}^{-1} K g_{j}, g_{j}\right)}{ }_{\#} \operatorname{Res}_{H \cap g_{j}^{-1} K g_{j}}^{H} \alpha\right)(t) \\
& =\sum\left\{g \otimes \ell_{\left(H \cap g_{j}^{-1} K g_{j}, g_{j}\right)} \operatorname{Res}_{H \cap g_{j}^{-1} K g_{j}}^{H} \alpha\left(t^{\prime}\right) \mid\right. \\
& \left.\left[g, t^{\prime}\right] \in K \times_{K \cap g_{j} H g_{j}^{-1}} \Theta_{K \cap g_{j} H g_{j}^{-1}}, g t^{\prime}=t\right\} \\
& =\sum\left\{g \otimes\left(e \otimes \alpha\left(g_{j}^{-1} t^{\prime}\right)\right) \mid\left[g, t^{\prime}\right] \in K \times_{K \cap g_{j} H g_{j}^{-1}} \Theta_{K \cap g_{j} H g_{j}^{-1}}, g t^{\prime}=t\right\} \\
& =\sum\left\{g \otimes\left(e \otimes \alpha\left(t^{\prime \prime}\right)\right) \mid\left[g g_{j}, t^{\prime \prime}\right] \in K g_{j} \times_{H \cap g_{j}^{-1} K g_{j}} \Theta_{H \cap g_{j}^{-1} K g_{j}}, g g_{j} t^{\prime \prime}=t\right\} \\
& \stackrel{\omega}{=} \sum\left\{g g_{j} \otimes \alpha\left(t^{\prime \prime}\right) \mid\left[g g_{j}, t^{\prime \prime}\right] \in K g_{j} \times_{H \cap g_{j}^{-1} K g_{j}} \Theta_{H \cap g_{j}^{-1} K g_{j}}, g g_{j} t^{\prime \prime}=t\right\} .
\end{aligned}
$$

The proposition follows immediately from these equalities. 


\section{Positioned Hermitian $R[G]$-modules}

In this section we introduce the Grothendieck-Witt rings of $\Theta$-positioned Hermitian $R[G]$-modules.

Definition 4.1. Let $M$ be an $R[G]$-module. A map $B: M \times M \rightarrow R$ is called a Hermitian form on $M$ if the following conditions (1)-(3) are satisfied:

(1) $B$ is $R$-bilinear,

(2) $B$ is $G$-invariant, namely $B(g x, g y)=B(x, y)$,

(3) $B$ is symmetric, namely $B(x, y)=B(y, x)$,

for all $x, y \in M$ and $g \in G$. A couple $(M, B)$ consisting of an $R[G]$-module $M$ and a Hermitian form $B$ on $M$ is called a Hermitian $R[G]$-module (or simply Hermitian module).

A Hermitian $R[G]$-module $(M, B)$ such that $M$ is a free $R$-module is said to be nonsingular if the associated map

$$
M \longrightarrow M^{\#}=\operatorname{Hom}_{R}(M, R) ; x \mapsto B(x,-)
$$

is bijective.

Let $H$ and $K$ be finite groups and $\varphi: H \rightarrow K$ a monomorphism. A Hermitian $R[K]$-module $(N, B)$ yields a Hermitian $R[H]$-module $\left(\varphi^{\#} N, \varphi^{\#} B\right)$ in a canonical way. By definition $\varphi^{\#} N$ is nothing but $N$ as an $R$-module. The map $\varphi^{\#} B$ : $\varphi^{\#} N \times \varphi^{\#} N \rightarrow R$ is also the same as $B: N \times N \rightarrow R$. Clearly, $\varphi^{\#} B$ is $R$-bilinear and symmetric. It is obvious that if $B$ is nonsingular, then so is $\varphi^{\#} B$. Since

$$
\varphi^{\#} B(h x, h y)=B(\varphi(h) x, \varphi(h) y)=B(x, y)
$$

for $h \in H, x, y \in \varphi^{\#} N$, it follows that $\varphi^{\#} B$ is $H$-invariant.

Proposition 4.2. Let $\varphi: H \rightarrow K$ be a monomorphism and let $\left(N_{i}, B_{i}\right), i=1,2$, be Hermitian $R[K]$-modules. Then

$$
\left(\varphi^{\#} N_{1} \otimes_{R} \varphi^{\#} N_{2}, \varphi^{\#} B_{1} \otimes_{R} \varphi^{\#} B_{2}\right)=\left(\varphi^{\#}\left(N_{1} \otimes_{R} N_{2}\right), \varphi^{\#}\left(B_{1} \otimes B_{2}\right)\right) .
$$

This proposition is obviously true.

Let $(M, B)$ be a Hermitian $R[H]$-module. Then, by definition,

$$
\varphi_{\#} M=R[K] \otimes_{R[H], \varphi} M .
$$

We define the $R$-bilinear form

$$
\varphi_{\#} B: \varphi_{\#} M \times \varphi_{\#} M \rightarrow R
$$

so that

$$
\varphi_{\#} B\left(a \otimes_{\varphi} x, b \otimes_{\varphi} y\right)=\delta_{a \varphi(H), b \varphi(H)} B\left(x, \varphi^{-1}\left(a^{-1} b\right) y\right),
$$

for $a, b \in K$ and $x, y \in M$, where $\delta_{a \varphi(H), b \varphi(H)}=1$ if $a \varphi(H)=b \varphi(H)$, and $\delta_{a \varphi(H), b \varphi(H)}=0$ otherwise. It is clear that $\varphi_{\#} B$ is $K$-invariant and symmetric. If $B$ is nonsingular, then so is $\varphi_{\#} B$. 
Proposition 4.3. Let $H$ be a subgroup of $G, B$ a Hermitian form on an $R[H]$ module $M$, and $g$ an element of $G$. Then the diagram

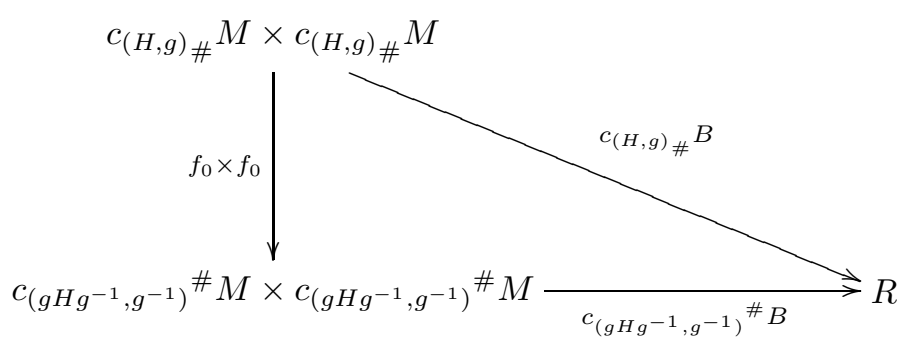

commutes, where $f_{0}$ is the canonical $R\left[g g^{-1}\right]$-isomorphism (cf. Proposition [3.2).

The proof is straightforward.

Proposition 4.4. Let $\varphi: H \rightarrow K$ be a monomorphism, and let $B$ and $B^{\prime}$ be Hermitian forms on an $R[H]$-module $M$ and an $R[K]$-module $N$, respectively. Then the following diagram commutes:

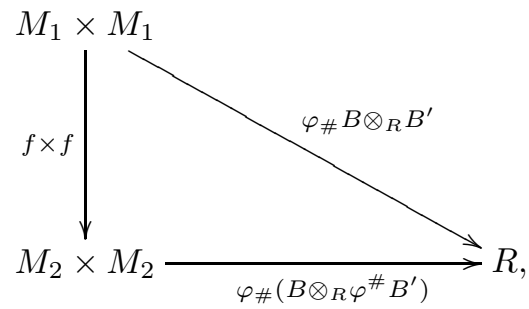

where $M_{1}=\left(R[K] \otimes_{R[H], \varphi} M\right) \otimes_{R} N, M_{2}=R[K] \otimes_{R[H], \varphi}\left(M \otimes_{R} \varphi^{\#} N\right)$, and $f$ is the canonical isomorphism (cf. Proposition 3.3).

Proof. The commutability follows from

$$
\begin{aligned}
\varphi_{\#} B \otimes_{R} B^{\prime}\left(\left(a \otimes_{\varphi} x\right) \otimes u,\left(b \otimes_{\varphi} y\right) \otimes v\right) & =\varphi_{\#} B\left(a \otimes_{\varphi} x,\left(b \otimes_{\varphi} y\right)\right) B^{\prime}(u, v) \\
& =\delta_{a \varphi(H), b \varphi(H)} B\left(x, \varphi^{-1}\left(a^{-1} b\right) y\right) B^{\prime}(u, v)
\end{aligned}
$$

and

$$
\begin{aligned}
\varphi_{\#}\left(B \otimes_{R} \varphi^{\#} B^{\prime}\right) & \left(a \otimes_{\varphi}\left(x \otimes a^{-1} u\right), b \otimes_{\varphi}\left(y \otimes b^{-1} v\right)\right) \\
& =\delta_{a \varphi(H), b \varphi(H)}\left(B \otimes_{R} \varphi^{\#} B^{\prime}\right)\left(x \otimes a^{-1} u, \varphi^{-1}\left(a^{-1} b\right)\left(y \otimes b^{-1} v\right)\right) \\
& =\delta_{a \varphi(H), b \varphi(H)} B\left(x, \varphi^{-1}\left(a^{-1} b\right) y\right) B^{\prime}\left(a^{-1} u, \varphi\left(\varphi^{-1}\left(a^{-1} b\right)\right) b^{-1} v\right) \\
& =\delta_{a \varphi(H), b \varphi(H)} B\left(x, \varphi^{-1}\left(a^{-1} b\right) y\right) B^{\prime}\left(a^{-1} u, a^{-1} v\right) \\
& =\delta_{a \varphi(H), b \varphi(H)} B\left(x, \varphi^{-1}\left(a^{-1} b\right) y\right) B^{\prime}(u, v),
\end{aligned}
$$

for $a, b \in K, x, y \in M$, and $u, v \in N$.

Proposition 4.5. Let $H$ be a subgroup of $G$ and $(M, B)$ a Hermitian $R[H]$-module. Then for any $g \in H$, the following diagrams commute:

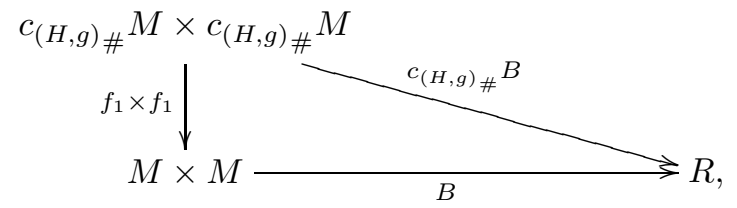




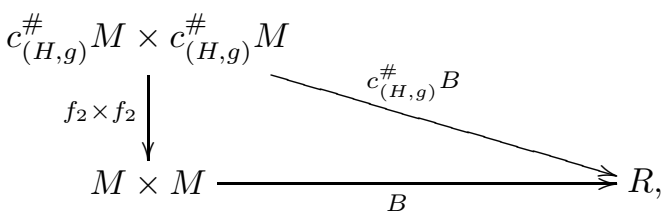

where $f_{1}$ and $f_{2}$ are the canonical isomorphisms (cf. Proposition 3.4).

Proof. The commutability of the first diagram follows from

$$
c_{(H, g)} B(e \otimes x, e \otimes y)=B(x, y)
$$

and

$$
B\left(f_{1}(e \otimes x), f_{1}(e \otimes y)\right)=B(g x, g y)=B(x, y) .
$$

The commutability of the second diagram follows from

$$
c_{(H, g)}{ }^{\#} B(x, y)=B(x, y)
$$

and

$$
B\left(f_{2}(x), f_{2}(y)\right)=B\left(g^{-1} x, g^{-1} y\right)=B(x, y) .
$$

Proposition 4.6. For any subgroups $H$ and $K$ of $G$, each Hermitian $R[H]$-module $(M, B)$ satisfies the Mackey double coset formula. Namely,

$$
\operatorname{Res}_{K}^{G} \operatorname{Ind}_{H}^{K} B=\bigoplus_{K g H \in K \backslash G / H} \operatorname{Ind}_{K \cap g H g^{-1}}^{K} c_{\left(H \cap g^{-1} K g, g\right)_{\#}} \operatorname{Res}_{H \cap g^{-1} K g}^{H} B .
$$

More precisely, the following diagram commutes:

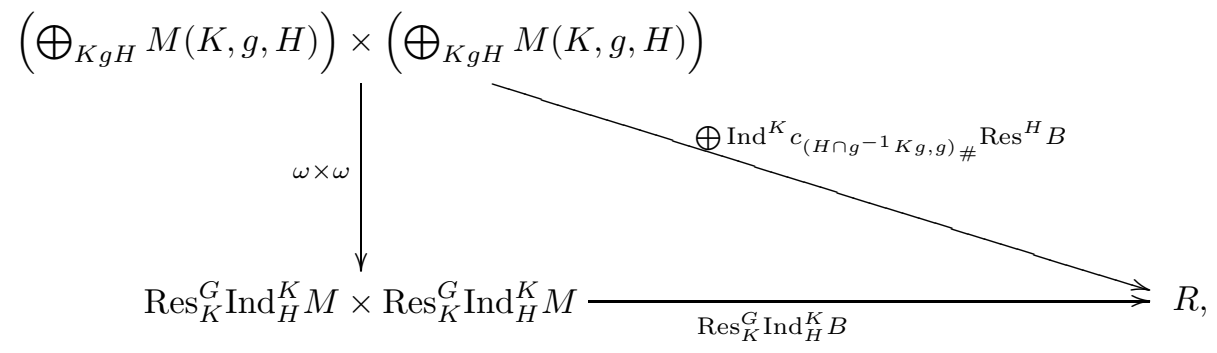

where $K g H$ runs over $K \backslash G / H$,

$$
M(K, g, H)=\operatorname{Ind}_{K \cap g g^{-1}}^{K} c_{\left(H \cap g^{-1} K g, g\right)} \operatorname{Res}_{H \cap g^{-1} K g}^{H} M,
$$

and $\omega$ is the canonical isomorphism (cf. Proposition 3.5).

Proof. For $u, v \in R[K] \otimes_{R\left[K \cap g H g^{-1}\right]} c_{\left(H \cap g^{-1} K g, g\right)} \operatorname{Res}_{H \cap g^{-1} K g}^{H} M$ with $u=a \otimes$ $(e \otimes x)$ and $v=b \otimes(e \otimes x)$ respectively, where $a, b \in K, x, y \in \operatorname{Res}_{H \cap g^{-1} K g}^{H} M$, we have

$$
\begin{aligned}
& \operatorname{Ind}_{K}^{K} \cap g H g^{-1} c_{\left(H \cap g^{-1} K g, g\right)_{\#}} \operatorname{Res}_{H \cap g^{-1} K g}^{H} B(u, v) \\
& \quad=\delta_{a\left(K \cap g H g^{-1}\right), b\left(K \cap g H g^{-1}\right)} c_{\left(H \cap g^{-1} K g, g\right)} \operatorname{Res}_{H \cap g^{-1} K g}^{H} B\left(e \otimes x, a^{-1} b(e \otimes y)\right) \\
& \quad=\delta_{a\left(K \cap g H g^{-1}\right), b\left(K \cap g H g^{-1}\right)} B\left(x, g^{-1} a^{-1} b g y\right)
\end{aligned}
$$


and

$$
\begin{aligned}
\operatorname{Res}_{K}^{G} \operatorname{Ind}_{H}^{G} B(a g \otimes x, b g \otimes y) & =\delta_{a g H, b g H} B\left(x,(a g)^{-1} b g y\right) \\
& =\delta_{a g H, b g H} B\left(x, g^{-1} a^{-1} b g y\right) \\
& =\delta_{a\left(K \cap g H g^{-1}\right), b\left(K \cap g H g^{-1}\right)} B\left(x, g^{-1} a^{-1} b g y\right) .
\end{aligned}
$$

Thus we obtain the proposition.

Definition 4.7. Let $\Theta$ be a finite $G$-set. A triple $(M, B, \alpha)$ consisting of a Hermitian $R[G]$-module $(M, B)$ and a $G$-map $\alpha: \Theta \rightarrow M$ is called a $\Theta$-positioned Hermitian $R[G]$-module (or simply $\Theta$-positioned Hermitian module).

Let $\mathcal{H}(R, G, \Theta)$ stand for the family of all $\Theta$-positioned Hermitian $R[G]$-modules $(M, B, \alpha)$ such that $M$ is an $R$-free $R[G]$-module and $B: M \times M \rightarrow R$ is nonsingular. We say that $\alpha$ is totally isotropic (resp. trivial) if $B(\operatorname{Im}(\alpha), \operatorname{Im}(\alpha))=0$ (resp. $\operatorname{Im}(\alpha)=0)$. We set

$$
\begin{aligned}
& \mathcal{H}(R, G, \Theta)^{\text {t-iso }}=\{(M, B, \alpha) \in \mathcal{H}(R, G, \Theta) \mid \alpha \text { is totally isotropic }\}, \\
& \mathcal{H}(R, G, \Theta)^{\text {triv }}=\{(M, B, \alpha) \in \mathcal{H}(R, G, \Theta) \mid \alpha \text { is trivial }\} .
\end{aligned}
$$

Let

$$
\mathrm{KH}_{0}(R, G, \Theta), \quad \mathrm{KH}_{0}(R, G, \Theta)^{\mathrm{t} \text {-iso }} \text { and } \mathrm{KH}_{0}(R, G)
$$

denote the Grothendieck groups of $\mathcal{H}(R, G, \Theta), \mathcal{H}(R, G, \Theta)^{\text {t-iso }}$ and $\mathcal{H}(R, G, \Theta)^{\text {triv }}$, respectively, under orthogonal sum.

Let $\boldsymbol{M}=(M, B, \alpha)$ be an object in $\mathcal{H}(R, G, \Theta)$. An $R$-direct summand, $R[G]$ submodule $U$ of $M$ is called a Quillen submodule of $M$ if $U \subset U^{\perp}$ and $\operatorname{Im}(\alpha) \subset U$ both hold, where

$$
U^{\perp}=\{x \in M \mid B(x, y)=0(\forall y \in U)\} .
$$

In this case, $(\boldsymbol{M}, U)$ is called a Quillen pair. If $\boldsymbol{M} \in \mathcal{H}(R, G, \Theta)$ admits a Quillen submodule, then $\boldsymbol{M}$ belongs to $\mathcal{H}(R, G, \Theta)^{\mathrm{t} \text {-iso }}$ by definition. For a Quillen pair $(\boldsymbol{M}, U)$, we have the well-defined map

$$
B^{\perp}: U^{\perp} / U \times U^{\perp} / U \rightarrow R ; B^{\perp}(x+U, y+U)=B(x, y) \quad\left(x, y \in U^{\perp}\right) .
$$

Proposition 4.8. Let $(\boldsymbol{M}, U)$, where $\boldsymbol{M}=(M, B, \alpha)$, be a Quillen pair. Then $U^{\perp} / U$ is an $R$-free $R[G]$-module and $B^{\perp}$ is a nonsingular Hermitian form on $U^{\perp} / U$.

Proof. Since $U$ is an $R$-direct summand of $M, M$ factors to $M=U \oplus N$ as $R$ modules. It follows that $U$ and $N$ both are $R$-free, and so are $U^{\#}=\operatorname{Hom}_{R}(U, R)$ and $M / U$. Thus, the exact sequence

$$
0 \longrightarrow U^{\perp} / U \longrightarrow M / U \longrightarrow U^{\#} \longrightarrow 0
$$

splits via $R$-homomorhisms, and hence $U^{\perp} / U$ is an $R$-direct summand of $M / U$. In particular, $U^{\perp} / U$ is $R$-free.

It is obvious that $B^{\perp}$ is $R$-bilinear, $G$-invariant and symmetric. So, it suffices to prove that $B^{\perp}$ is nonsingular. Since $B$ is nonsingular, we can take an $R$-basis

$$
\left\{u_{1}, \ldots, u_{m}, y_{1}, \ldots, y_{n}, v_{1}, \ldots, v_{m}\right\}
$$

of $M$ so that $\left\{u_{1}, \ldots, u_{m}\right\}$ is an $R$-basis of $U, y_{j} \in U^{\perp}$, and $B\left(v_{i}, u_{j}\right)=\delta_{i, j}$ and $B\left(v_{i}, y_{j}\right)=0$, where $\delta_{i, j}=1$ if $i=j$ and $\delta_{i, j}=0$ otherwise. Let $V$ denote the $R$ submodule of $M$ generated by $\left\{v_{1}, \ldots, v_{m}\right\}$. There exist elements $z_{1}, \ldots, z_{n}$ of $M$ 
such that $B\left(z_{i}, u_{j}\right)=0, B\left(z_{i}, y_{j}\right)=\delta_{i, j}$ and $B\left(z_{i}, v_{j}\right)=0$. Write $z_{i}$ as $z_{i}=y_{i}^{\prime}+v_{i}^{\prime}$ with $y_{i}^{\prime} \in U^{\perp}$ and $v_{i}^{\prime} \in V$. Then

$$
B\left(y_{i}^{\prime}, y_{j}\right)=B\left(y_{i}^{\prime}+v_{i}^{\prime}, y_{j}\right)=B\left(z_{i}, y_{j}\right)=\delta_{i, j} .
$$

This shows that $B^{\perp}: U^{\perp} / U \times U^{\perp} / U \rightarrow R$ is nonsingular.

By the proposition, a Quillen pair $(\boldsymbol{M}, U)$ induces an object $\left(U^{\perp} / U, B^{\perp}\right.$, triv) of $\mathcal{H}(R, G, \Theta)$, where triv : $\Theta \rightarrow U^{\perp} / U$ is the trivial map.

We define the Grothendieck-Witt groups

$$
\mathrm{GW}_{0}(R, G, \Theta), \quad \mathrm{GW}_{0}(R, G, \Theta)^{\mathrm{t}-\text { iso }}, \quad \mathrm{GW}_{0}(R, G)
$$

by

$$
\begin{aligned}
& \operatorname{GW}_{0}(R, G, \Theta)=\mathrm{KH}_{0}(R, G, \Theta) /\left\langle[\boldsymbol{M}]-\left[U^{\perp} / U, B^{\perp}, \operatorname{triv}\right]\right\rangle, \\
& \mathrm{GW}_{0}(R, G, \Theta)^{\mathrm{t} \text {-iso }}=\mathrm{KH}_{0}(R, G, \Theta)^{\mathrm{t}-\text { iso }} /\left\langle[\boldsymbol{M}]-\left[U^{\perp} / U, B^{\perp}, \operatorname{triv}\right]\right\rangle, \\
& \mathrm{GW}_{0}(R, G)=\mathrm{KH}_{0}(R, G) /\left\langle[\boldsymbol{M}]-\left[U^{\perp} / U, B^{\perp}, \operatorname{triv}\right]\right\rangle,
\end{aligned}
$$

where $(\boldsymbol{M}, U)$ ranges over all Quillen pairs in $\mathcal{H}(R, G, \Theta), \mathcal{H}(R, G, \Theta)^{\text {t-iso }}$ and $\mathcal{H}(R, G, \Theta)^{\text {triv }}$, respectively. By definition, there are canonical homomorphisms

$$
\mathrm{GW}_{0}(R, G) \rightarrow \mathrm{GW}_{0}(R, G, \Theta)^{\mathrm{t} \text {-iso }}
$$

and

$$
\mathrm{GW}_{0}(R, G, \Theta)^{\mathrm{t} \text {-iso }} \rightarrow \mathrm{GW}_{0}(R, G, \Theta) .
$$

Proposition 4.9. The homomorphisms

$$
\mathrm{GW}_{0}(R, G) \rightarrow \mathrm{GW}_{0}(R, G, \Theta)^{\mathrm{t} \text {-iso }} \text { and } \mathrm{GW}_{0}(R, G, \Theta)^{\mathrm{t} \text {-iso }} \rightarrow \mathrm{GW}_{0}(R, G, \Theta)
$$

are both injective. Moreover, the homomorphism $\operatorname{GW}_{0}(R, G) \rightarrow \mathrm{GW}_{0}(R, G, \Theta)^{\mathrm{t} \text {-iso }}$ is an isomorphism.

Proof. Consider the homomorphism

$$
\mathrm{GW}_{0}(R, G, \Theta) \rightarrow \mathrm{GW}_{0}(R, G)
$$

assigning $[M, B$, triv $]$ to $[M, B, \alpha]$. Since the composition

$$
\mathrm{GW}_{0}(R, G) \rightarrow \mathrm{GW}_{0}(R, G, \Theta)^{\text {t-iso }} \rightarrow \mathrm{GW}_{0}(R, G, \Theta) \rightarrow \mathrm{GW}_{0}(R, G)
$$

is the identity map, the homomorphisms

$$
\mathrm{GW}_{0}(R, G) \rightarrow \mathrm{GW}_{0}(R, G, \Theta)^{\mathrm{t} \text {-iso }} \text { and } \mathrm{GW}_{0}(R, G) \rightarrow \mathrm{GW}_{0}(R, G, \Theta)
$$

are injective.

Let $\boldsymbol{M}=(M, B, \alpha)$ be a $\Theta$-positioned $R[G]$-Hermitian module such that $\alpha$ is totally isotropic. Then, let $L$ denote the $R[G]$-submodule of $M$ generated by $\alpha(\Theta)$, and set

$$
U=\{x \in M \mid r x \in L \text { for some } r \in R \text { with } r \neq 0\} .
$$

Then $B(U, U)=0$, and $U$ is an $R$-direct summand, $R[G]$-submodule of $M$. Thus, we have

$$
[M, B, \alpha]=\left[U^{\perp} / U, B^{\perp}, \text { triv }\right] \text { in } G W_{0}(R, G, \Theta)^{\mathrm{t} \text {-iso }} .
$$

This implies that the canonical homomorphism $\mathrm{GW}_{0}(R, G) \rightarrow \mathrm{GW}_{0}(R, G, \Theta)^{\mathrm{t} \text {-iso }}$ is surjective.

For $\Theta$-positioned Hermitian $R[G]$-modules $\boldsymbol{M}_{1}=\left(M_{1}, B_{1}, \alpha_{1}\right)$ and $\boldsymbol{M}_{2}=\left(M_{2}\right.$, $\left.B_{2}, \alpha_{2}\right)$, we define the tensor product $\boldsymbol{M}_{1} \otimes_{R} \boldsymbol{M}_{2}$ over $R$ as the $\Theta$-positioned Hermitian $R[G]$-module $\left(M_{1} \otimes_{R} M_{2}, B_{1} \otimes_{R} B_{2}, \alpha_{1} \otimes_{R} \alpha_{2}\right)$. 
Proposition 4.10. Let $\Theta$ be a finite $G$-set. Then $\mathrm{GW}_{0}(R, G, \Theta)$ and $\mathrm{GW}_{0}(R, G)$ $\left(=\mathrm{GW}_{0}(R, G, \Theta)^{\mathrm{t}-\mathrm{iso}}\right)$ are commutative rings under the multiplication induced from the tensor product over $R$. Moreover, the rings $\mathrm{GW}_{0}(R, G, \Theta)$ and $\mathrm{GW}_{0}(R, G)$ possess units. Actually, the units of $\mathrm{GW}_{0}(R, G, \Theta)$ and $\mathrm{GW}_{0}(R, G)$ are the equivalence classes of

$$
(R, B: R \times R \rightarrow R, \alpha: \Theta \rightarrow R) \text { and }(R, B: R \times R \rightarrow R \text {, triv }: \Theta \rightarrow R) \text {, }
$$

respectively, where $G$ acts trivially on $R, B$ is the map defined by $B\left(r_{1}, r_{2}\right)=r_{1} r_{2}$ for $r_{1}, r_{2} \in R$, and $\alpha$ is the map defined by $\alpha(t)=1$ for $t \in \Theta$.

\section{The special Grothendieck-Witt Rings}

Let $S$ be a conjugation-invariant subset of

$$
G(2)=\left\{g \in G \mid g^{2}=e, g \neq e\right\}
$$

and let $\mathfrak{P}(S)$ denote the set of all subsets of $S$. Then the $G$-action on $S$ by conjugation yields a $G$-action on $\mathfrak{P}(S)$. Let $\Theta$ be a finite $G$-set and $\rho^{(2)}: \Theta \rightarrow \mathfrak{P}(S)$ a $G$-map.

For a $G$-map $\alpha: \Theta \rightarrow M$, where $M$ is an $R[G]$-module, we define the map $\Delta_{\alpha}: S \rightarrow M$ by

$$
\Delta_{\alpha}(s)=\sum_{t}\left\{\alpha(t) \mid t \in \Theta, \rho^{(2)}(t) \ni s\right\} \quad(s \in S) .
$$

Proposition 5.1. The map $\Delta_{\alpha}$ above is a G-map, namely $\Delta_{\alpha}\left(g s g^{-1}\right)=g \Delta_{\alpha}(s)$ for $g \in G$ and $s \in S$.

Proof. The proof runs as follows:

$$
\begin{aligned}
g \Delta_{\alpha}(s) & =g \sum_{t}\left\{\alpha(t) \mid t \in \Theta, \rho^{(2)}(t) \ni s\right\} \\
& =\sum_{t}\left\{\alpha(g t) \mid t \in \Theta, \rho^{(2)}(t) \ni s\right\} \\
& =\sum_{t^{\prime}}\left\{\alpha\left(t^{\prime}\right) \mid g^{-1} t^{\prime} \in \Theta, \rho^{(2)}\left(g^{-1} t^{\prime}\right) \ni s\right\} \\
& =\sum_{t^{\prime}}\left\{\alpha\left(t^{\prime}\right) \mid t^{\prime} \in \Theta, \rho^{(2)}\left(t^{\prime}\right) \ni g s g^{-1}\right\} \\
& =\Delta_{\alpha}\left(g s g^{-1}\right) .
\end{aligned}
$$

Let $\boldsymbol{M}=(M, B, \alpha)$ be an object in $\mathcal{H}(R, G, \Theta)$. We introduce a map

$$
\nabla_{\boldsymbol{M}}: M \rightarrow \operatorname{Map}(S, R / 2 R)
$$

which plays a key role in this paper. Define $\nabla_{\boldsymbol{M}}(x)(s) \in R / 2 R$ for $x \in M$ and $s \in S$ by

$$
\nabla_{\boldsymbol{M}}(x)(s)=B\left(\Delta_{\alpha}(s)-x, s x\right) .
$$

Proposition 5.2. The map $\nabla_{\boldsymbol{M}}: M \rightarrow \operatorname{Map}(S, R / 2 R)$ is a $\mathbb{Z}[G]$-homomorphism. Namely, the following hold:

(1) $\nabla_{\boldsymbol{M}}(x+y)(s)=\nabla_{\boldsymbol{M}}(x)(s)+\nabla_{\boldsymbol{M}}(y)(s) \quad(x, y \in M, s \in S)$,

(2) $\nabla_{\boldsymbol{M}}(g x)(s)=\nabla_{\boldsymbol{M}}(x)\left(g^{-1} s g\right) \quad(x \in M, s \in S)$.

If $R$ is square identical, $\nabla_{\boldsymbol{M}}: M \rightarrow \operatorname{Map}(S, R / 2 R)$ is an $R[G]$-homomorphism. 
Proof. The formula (1) is obtained as follows:

$$
\begin{aligned}
\nabla_{\boldsymbol{M}}(x+y)(s) & =B\left(\Delta_{\alpha}(s)-(x+y), s(x+y)\right) \\
& =\nabla_{\boldsymbol{M}}(x)(s)+\nabla_{\boldsymbol{M}}(y)(s)+B(-x, s y)+B(-y, s x) \\
& =\nabla_{\boldsymbol{M}}(x)(s)+\nabla_{\boldsymbol{M}}(y)(s)-(B(x, s y)+B(y, s x)) \\
& =\nabla_{\boldsymbol{M}}(x)(s)+\nabla_{\boldsymbol{M}}(y)(s) \quad \text { in } R / 2 R .
\end{aligned}
$$

The formula (2) holds because

$$
\begin{aligned}
\nabla_{\boldsymbol{M}}(g x)(s) & =B\left(\Delta_{\alpha}(s)-g x, s g x\right) \\
& =B\left(g^{-1} \Delta_{\alpha}(s)-x, g^{-1} s g x\right) \\
& =B\left(\Delta_{\alpha}\left(g^{-1} s g\right)-x, g^{-1} s g x\right) \\
& =\nabla_{\boldsymbol{M}}(x)\left(g^{-1} s g\right) \quad \text { in } R / 2 R .
\end{aligned}
$$

The last assertion in the proposition is true since

$$
\begin{aligned}
\nabla_{\boldsymbol{M}}(r x)(s) & =B\left(\Delta_{\alpha}(s)-r x, s r x\right) \\
& =B\left(\Delta_{\alpha}(s), s r x\right)-B(r x, s r x) \\
& =r B\left(\Delta_{\alpha}(s), s x\right)-r^{2} B(x, s x) \\
& =r B\left(\Delta_{\alpha}(s), s x\right)-r B(x, s x) \\
& =r B\left(\Delta_{\alpha}(s)-x, s x\right) \\
& =r \nabla_{\boldsymbol{M}}(x)(s) \quad \text { in } R / 2 R .
\end{aligned}
$$

We have established the proposition above.

Let $\mathcal{S H}(R, G, S, \Theta), \mathcal{S H}(R, G, S, \Theta)^{\mathrm{t}-\text { iso }}$ and $\mathcal{S H}(R, G, S, \Theta)^{\text {triv }}$ denote the family consisting of objects $M$ with $\nabla_{\boldsymbol{M}}=0$ of $\mathcal{H}(R, G, \Theta), \mathcal{H}(R, G, \Theta)^{\mathrm{t} \text {-iso }}$ and $\mathcal{H}(R, G, \Theta)^{\text {triv }}$, respectively. We denote the Grothendieck groups of these under orthogonal sum by

$$
\mathrm{KSH}_{0}(R, G, S, \Theta), \mathrm{KSH}_{0}(R, G, S, \Theta)^{\mathrm{t} \text {-iso }} \text { and } \mathrm{KSH}_{0}(R, G, S) \text {, }
$$

respectively. Moreover, we define the special Grothendieck-Witt groups

$$
\operatorname{SGW}_{0}(R, G, S, \Theta), \quad \operatorname{SGW}_{0}(R, G, S, \Theta)^{\mathrm{t} \text {-iso }}, \quad \operatorname{SGW}_{0}(R, G, S)
$$

by

$$
\begin{aligned}
& \operatorname{SGW}_{0}(R, G, S, \Theta)=\operatorname{KSH}_{0}(R, G, S, \Theta) /\left\langle[\boldsymbol{M}]-\left[U^{\perp} / U, B^{\perp}, \text { triv }\right]\right\rangle, \\
& \operatorname{SGW}_{0}(R, G, S, \Theta)^{\mathrm{t} \text {-iso }}=\mathrm{KSH}_{0}(R, G, S, \Theta)^{\mathrm{t} \text {-iso }} /\left\langle[\boldsymbol{M}]-\left[U^{\perp} / U, B^{\perp}, \text { triv }\right]\right\rangle, \\
& \operatorname{SGW}_{0}(R, G, S)=\mathrm{KSH}_{0}(R, G, S) /\left\langle[\boldsymbol{M}]-\left[U^{\perp} / U, B^{\perp}, \text { triv }\right]\right\rangle,
\end{aligned}
$$

where $(\boldsymbol{M}, U)$ ranges over all Quillen pairs in $\mathcal{S H}(R, G, S, \Theta), \mathcal{S H}(R, G, S, \Theta)^{\mathrm{t} \text {-iso }}$ and $\mathcal{S H}(R, G, S, \Theta)^{\text {triv }}$, respectively. Here we remark that if $\boldsymbol{M} \in \mathcal{S H}(R, G, S, \Theta)$ admits a Quillen submodule, then $\boldsymbol{M}$ belongs to $\mathcal{S H}(R, G, S, \Theta)^{\mathrm{t} \text {-iso }}$. By definition, there are canonical homomorphisms

$$
\mathrm{SGW}_{0}(R, G, S) \rightarrow \mathrm{SGW}_{0}(R, G, S, \Theta)^{\mathrm{t} \text {-iso }}
$$

and

$$
\mathrm{SGW}_{0}(R, G, S, \Theta)^{\mathrm{t} \text {-iso }} \rightarrow \mathrm{SGW}_{0}(R, G, S, \Theta) .
$$


Proposition 5.3. The homomorphism $\mathrm{SGW}_{0}(R, G, S) \rightarrow \mathrm{SGW}_{0}(R, G, S, \Theta)^{\mathrm{t} \text {-iso }}$ is surjective, and the homomorphism $\mathrm{SGW}_{0}(R, G, S, \Theta)^{\mathrm{t} \text {-iso }} \rightarrow \operatorname{SGW}_{0}(R, G, S, \Theta)$ is injective.

Proof. The proof of the surjectivity of $\operatorname{SGW}_{0}(R, G, S) \rightarrow \operatorname{SGW}_{0}(R, G, S, \Theta)^{\text {t-iso }}$ is the same as that of $\mathrm{GW}_{0}(R, G) \rightarrow \mathrm{GW}_{0}(R, G, \Theta)^{\mathrm{t} \text {-iso }}$ (see Proposition 4.9).

Let $\boldsymbol{M}$ be an object of $\mathcal{S H}(R, G, S, \Theta)^{\mathrm{t}-\text { iso }}$ such that $[\boldsymbol{M}]=0$ in $\operatorname{SGW}_{0}(R, G, S, \Theta)$. Then there exist objects $\boldsymbol{M}^{\prime}=\left(M^{\prime}, B^{\prime}, \alpha^{\prime}\right), \boldsymbol{M}_{1}=\left(M_{1}, B_{1}, \alpha_{1}\right)$ with a Quillen submodule $U_{1}$, and $\boldsymbol{M}_{2}=\left(M_{2}, B_{2}, \alpha_{2}\right)$ with a Quillen submodule $U_{2}$ of $\mathcal{S H}(R, G, S, \Theta)$ such that

$$
\boldsymbol{M} \oplus \boldsymbol{M}^{\prime} \oplus \boldsymbol{M}_{1} \oplus\left(U_{2}^{\perp} / U_{2}, B_{2}^{\perp}, \text { triv }\right) \cong \boldsymbol{M}^{\prime} \oplus \boldsymbol{M}_{2} \oplus\left(U_{1}^{\perp} / U_{1}, B_{1}^{\perp}, \text { triv }\right) .
$$

By definition, both $\boldsymbol{M}_{1}$ and $\boldsymbol{M}_{2}$ belong to $\mathcal{S H}(R, G, S, \Theta)^{\mathrm{t} \text {-iso }}$. The object $\boldsymbol{M}^{\prime}$ above may be replaced by

$$
M^{\prime \prime}=\left(M^{\prime}, B^{\prime}, \alpha^{\prime}\right) \oplus\left(M^{\prime},-B^{\prime},-\alpha^{\prime}\right) .
$$

Then $\boldsymbol{M}^{\prime \prime}$ has the Quillen submodule

$$
U^{\prime \prime}=\left\{(x, x) \in M^{\prime} \oplus M^{\prime} \mid x \in M^{\prime}\right\}
$$

and hence belongs to $\mathcal{S H}(R, G, S, \Theta)^{\text {t-iso }}$, which lets us conclude that

$$
[\boldsymbol{M}]=0 \text { in } \mathrm{SGW}_{0}(R, G, S, \Theta)^{\mathrm{t} \text {-iso }} .
$$

Proposition 5.4. If, for each $s \in S$, there is at most one element $t \in \Theta$ such that $\rho^{(2)}(t) \ni s$, then $\mathrm{SGW}_{0}(R, G, S, \Theta), \mathrm{SGW}_{0}(R, G, S, \Theta)^{\mathrm{t}-\text { iso }}$ and $\mathrm{SGW}_{0}(R, G, S)$ are commutative rings, possibly without unit. If $R$ is square identical, and for each $s \in S$ there exists exactly one element $t \in \Theta$ such that $\rho^{(2)}(t) \ni s$, then $\operatorname{SGW}_{0}(R, G, S, \Theta)$ is a commutative ring with unit.

Proof. Let $\boldsymbol{M}_{1}=\left(M_{1}, B_{1}, \alpha_{1}\right)$ and $\boldsymbol{M}_{2}=\left(M_{2}, B_{2}, \alpha_{2}\right)$ be objects of $\mathcal{H}(R, G, \Theta)$ and $\mathcal{S H}(R, G, S, \Theta)$, respectively. Then

$$
\begin{aligned}
\nabla_{\boldsymbol{M}_{1} \otimes_{R} \boldsymbol{M}_{2}}( & \left.x_{1} \otimes x_{2}\right)(s)=B_{1} \otimes_{R} B_{2}\left(\Delta_{\alpha_{1} \otimes_{R} \alpha_{2}}(s)-x_{1} \otimes x_{2}, s\left(x_{1} \otimes x_{2}\right)\right) \\
= & B_{1} \otimes_{R} B_{2}\left(\Delta_{\alpha_{1}}(s) \otimes \Delta_{\alpha_{2}}(s)-x_{1} \otimes x_{2}, s x_{1} \otimes s x_{2}\right) \\
= & B_{1}\left(\Delta_{\alpha_{1}}(s), s x_{1}\right) B_{2}\left(\Delta_{\alpha_{2}}(s), s x_{2}\right)-B_{1}\left(x_{1}, s x_{1}\right) B_{2}\left(x_{2}, s x_{2}\right) \\
= & B_{1}\left(\Delta_{\alpha_{1}}(s)-x_{1}, s x_{1}\right) B_{2}\left(\Delta_{\alpha_{2}}(s), s x_{2}\right) \\
& +B_{1}\left(x_{1}, s x_{1}\right) B_{2}\left(\Delta_{\alpha_{2}}(s)-x_{2}, s x_{2}\right) \\
= & \nabla_{\boldsymbol{M}_{1}}\left(x_{1}\right)(s) B_{2}\left(\Delta_{\alpha_{2}}(s), s x_{2}\right)+B_{1}\left(x_{1}, s x_{1}\right) \nabla_{\boldsymbol{M}_{2}}\left(x_{2}\right)(s) \\
= & \nabla_{\boldsymbol{M}_{1}}\left(x_{1}\right)(s) B_{2}\left(\Delta_{\alpha_{2}}(s), s x_{2}\right) \quad \text { in } R / 2 R .
\end{aligned}
$$

By using this and Proposition 5.2 (1), we can show that the product $\boldsymbol{M}_{1} \otimes_{R} \boldsymbol{M}_{2}$ belongs to $\mathcal{S H}(R, G, S, \Theta)$ if $\boldsymbol{M}_{1}$ does. Therefore, the special Grothendieck-Witt groups are commutative rings.

Next we shall prove the last claim in the proposition. Let $(R, B, \alpha)$ denote the object in $\mathcal{H}(R, G, \Theta)$ such that $G$ acts trivially on $R, B\left(r_{1}, r_{2}\right)=r_{1} r_{2}\left(r_{1}, r_{2} \in R\right)$ and $\alpha(t)=1(t \in \Theta)$. Then, the associated $\nabla: M \rightarrow \operatorname{Map}(S, R / 2 R)$ is trivial, since

$$
\nabla(x)(s)=B(1-r, s r)=r-r^{2}=0 \quad \text { in } R / 2 R .
$$

Thus, $(R, B, \alpha)$ belongs to $\mathcal{S H}(R, G, S, \Theta)$, and therefore we can now conclude that the ring $\mathrm{SGW}_{0}(R, G, S, \Theta)$ possesses a unit. 
Proposition 5.5. The group $\mathrm{SGW}_{0}(R, G, S)$ is a module over the ring $\mathrm{GW}_{0}(R, G)$.

Proof. Let $\boldsymbol{M}_{1}=\left(M_{1}, B_{1}\right.$, triv $)$ and $\boldsymbol{M}_{2}=\left(M_{2}, B_{2}\right.$, triv $)$ be arbitrary objects of $\mathcal{H}(R, G, \Theta)^{\text {triv }}$ and $\mathcal{S H}(R, G, S, \Theta)^{\text {triv }}$, respectively. Then, as in the proof of Proposition 5.4 we have

$$
\nabla_{\boldsymbol{M}_{1} \otimes \boldsymbol{M}_{2}}\left(x_{1} \otimes x_{2}\right)(s)=\nabla_{\boldsymbol{M}_{1}}\left(x_{1}\right)(s) B_{2}\left(\Delta_{\alpha_{2}}(s), s x_{2}\right)+B_{1}\left(x_{1}, s x_{1}\right) \nabla_{\boldsymbol{M}_{2}}\left(x_{2}\right)(s) .
$$

Since $\Delta_{\alpha_{2}}(s)=0$ and $\nabla_{\boldsymbol{M}_{2}}\left(x_{2}\right)(s)=0, \nabla_{\boldsymbol{M}_{1} \otimes \boldsymbol{M}_{2}}$ vanishes. Thus $\boldsymbol{M}_{1} \otimes \boldsymbol{M}_{2}$ belongs to $\mathcal{S H}(R, G, S, \Theta)^{\text {triv }}$.

\section{6. $R[G]$-valued $\lambda$-Hermitian Forms}

Let $\lambda$ stand for 1 or -1 and let $w: G \rightarrow\{-1,1\}$ be a homomorphism. The group ring $A=R[G]$ is equipped with the anti-involution - defined by

$$
\overline{\sum_{g \in G} r_{g} g}=\sum_{g \in G} w(g) r_{g} g^{-1} \quad\left(r_{g} \in R\right) .
$$

Definition 6.1. Let $M$ be an $R[G]$-module. A map $B: M \times M \rightarrow R[G]$ is called an $R[G]$-valued $\lambda$-Hermitian form (or $\lambda$-Hermitian form) on $M$ if the following conditions (1) $-(3)$ are satisfied:

(1) $B$ is $R$-bilinear,

(2) $B(a x, b y)=b B(y, x) \bar{a}$,

(3) $B(x, y)=\lambda \overline{B(y, x)}$,

for all $x, y \in M, a, b \in R[G]$.

Let $B: M \times M \rightarrow R[G]$ be a $\lambda$-Hermitian form. For $x, y \in M, B(x, y)$ can be written as $\sum_{g \in G} B(x, y)_{g} g$ with $B(x, y)_{g} \in R$. Define the $R$-homomorphism $\varepsilon: R[G] \rightarrow R$ by

$$
\varepsilon\left(\sum_{g \in G} r_{g} g\right)=r_{e} \quad\left(r_{g} \in R\right) .
$$

Lemma 6.2. $B(x, y)_{g}=\varepsilon\left(B\left(x, g^{-1} y\right)\right)$ for all $x, y \in M$ and $g \in G$, and consequently

$$
B(x, y)=\sum_{g \in G} \varepsilon\left(B\left(x, g^{-1} y\right)\right) g .
$$

Proof. By definition, we have $B(x, y)_{e}=\varepsilon(B(x, y))$. By observing the coefficients of $g$ in $B(x, y)$ and

$$
g B\left(x, g^{-1} y\right)=\sum_{h \in G} B\left(x, g^{-1} y\right)_{h} g h,
$$

we have $B\left(x, g^{-1} y\right)_{e}=B(x, y)_{g}$. Thus, $B(x, y)_{g}=\varepsilon\left(B\left(x, g^{-1} y\right)\right)$.

Lemma 6.3. Let $M$ be as above. Then the composition $\varepsilon \circ B: M \times M \rightarrow R$ is a $\lambda$-symmetric, $(G, w)$-invariant, $R$-bilinear form on $M$. Namely, the following hold:

(1) $\varepsilon\left(B\left(x+x^{\prime}, r y\right)\right)=r \varepsilon(B(x, y))+r \varepsilon\left(B\left(x^{\prime}, y\right)\right)$,

(2) $\varepsilon(B(x, y))=\lambda \varepsilon(B(y, x))$,

(3) $\varepsilon(B(g x, g y))=w(g) \varepsilon(B(x, y))$,

for any $r \in R, x, x^{\prime}, y \in M$ and $g \in G$. 
Proof. (1) The proof is straightforward.

(2) The equality follows from $B(x, y)=\lambda \overline{B(y, x)}$.

(3) By comparing the coefficients of $e$ in $B(x, g y)$ and $w(g) B\left(g^{-1} x, y\right)$ :

$$
\begin{aligned}
& B(x, g y)=\sum_{h \in G} B(x, g y)_{h} h, \\
& w(g) B\left(g^{-1} x, y\right)=\sum_{h \in G} w(g) B\left(g^{-1} x, y\right)_{h} h,
\end{aligned}
$$

we have $\varepsilon(B(x, g y))=w(g) \varepsilon\left(B\left(g^{-1} x, y\right)\right)$, which is equivalent to the equality (3).

An $R[G]$-valued $\lambda$-Hermitian form $B$ on an $R[G]$-projective module $M$ is said to be nonsingular if the associated map

$$
M \rightarrow \operatorname{Hom}_{R[G]}(M, R[G]) ; x \longmapsto B(x,-)
$$

is bijective.

Lemma 6.4. Let $B$ be an $R[G]$-valued $\lambda$-Hermitian form on an $R[G]$-projective module $M$. Then $B$ is nonsingular if and only if the induced $R$-bilinear form $\varepsilon \circ B$ : $M \times M \rightarrow R$ is nonsingular.

Let $H$ and $K$ be finite groups with homomorphisms $w_{H}: H \rightarrow\{-1,1\}$ and $w_{K}: K \rightarrow\{-1,1\}$, respectively. Let $\varphi: H \rightarrow K$ be a monomorphism such that $w_{K} \circ \varphi=w_{H}$. Let $N$ be an $R[K]$-module and $B: N \times N \rightarrow R[K]$ a $\lambda$-Hermitian form. We define the map $\varphi^{\#} B: \varphi^{\#} N \times \varphi^{\#} N \rightarrow R[H]$ by

$$
\varphi^{\#} B(x, y)=\sum_{h \in H} \varepsilon\left(B\left(x, \varphi(h)^{-1} y\right)\right) h \quad\left(x, y \in \varphi^{\#} N\right) .
$$

It immediately follows that $\varphi^{\#} B$ is an $R[H]$-valued $\lambda$-Hermitian form on $\varphi^{\#} N$. If $B$ is nonsingular, then so is $\varphi^{\#} B$. Next let $M$ be a stably free $R[H]$-module. Then

$$
\varphi_{\#} M=R[K] \otimes_{R[H], \varphi} M
$$

is clearly a stably $R[K]$-free module. Let $B: M \times M \rightarrow R[H]$ be a $\lambda$-Hermitian form. We define the $R$-bilinear map $\varphi_{\#} B: \varphi_{\#} M \times \varphi_{\#} M \rightarrow R[K]$ so that

$$
\varphi_{\#} B\left(a \otimes_{\varphi} x, b \otimes_{\varphi} y\right)=\sum_{k \in K} w_{K}(a) \delta_{a \varphi(H), k^{-1} b \varphi(H)} \varepsilon\left(B\left(x, \varphi^{-1}\left(a^{-1} k^{-1} b\right) y\right)\right) k
$$

for $a, b \in K, x, y \in M$.

Lemma 6.5. Let $\varphi_{\#} B$ be as above. Then

$$
\varphi_{\#} B\left(a \otimes_{\varphi} x, b \otimes_{\varphi} y\right)=b \varphi^{\prime}(B(x, y)) \bar{a},
$$

for $a, b \in K, x, y \in M$; and $\varphi_{\#} B$ is an $R[K]$-valued $\lambda$-Hermitian form on $\varphi_{\#} M$, where $\varphi^{\prime}: R[H] \rightarrow R[K]$ is the ring homomorphism canonically induced by $\varphi: H \rightarrow$ $K$. If $B$ is nonsingular, then so is $\varphi_{\#} B$. 
Proof. The formula in the lemma is true because

$$
\begin{aligned}
\varphi_{\#} B\left(a \otimes_{\varphi} x, b \otimes_{\varphi} y\right) & =\sum_{k \in K} w_{K}(a) \delta_{a \varphi(H), k^{-1} b \varphi(H)} \varepsilon\left(B\left(x, \varphi^{-1}\left(a^{-1} k^{-1} b\right) y\right)\right) k \\
& =b\left(\sum_{k \in K} \delta_{\varphi(H), a^{-1} k^{-1} b \varphi(H)} \varepsilon\left(B\left(x, \varphi^{-1}\left(a^{-1} k^{-1} b\right) y\right)\right) b^{-1} k a\right) \bar{a} \\
& =b\left(\sum_{k^{\prime} \in K} \delta_{\varphi(H), k^{\prime-1} \varphi(H)} \varepsilon\left(B\left(x, \varphi^{-1}\left(k^{\prime-1}\right) y\right)\right) k^{\prime}\right) \bar{a} \\
& =b \varphi^{\prime}\left(\sum_{k^{\prime} \in K} \delta_{\varphi(H), k^{\prime}-1} \varphi(H) \varepsilon\left(B\left(x, \varphi^{-1}\left(k^{\prime-1}\right) y\right)\right) \varphi^{-1}\left(k^{\prime}\right)\right) \bar{a} \\
& =b \varphi^{\prime}(B(x, y)) \bar{a} .
\end{aligned}
$$

One can check the latter claim in the lemma by using this formula.

Proposition 6.6. Let $H$ be a subgroup of $G, B$ an $R[H]$-valued $\lambda$-Hermitian form on an $R[H]$-module $M$, and $g$ an element of $G$. Provided $w_{H}=w_{g H g^{-1}} \circ c_{(H, g)}$, the diagram

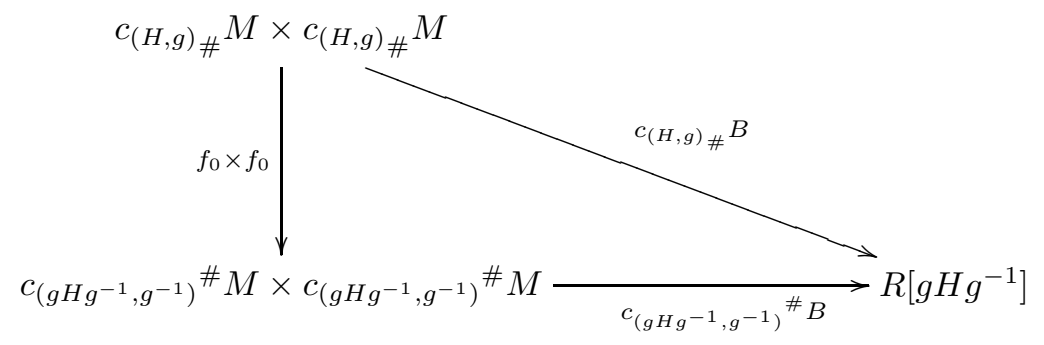

commutes, where $f_{0}$ is the canonical $R\left[g^{H} g^{-1}\right]$-isomorphism (cf. Proposition [3.2).

The proof of the proposition is straightforward.

Given a datum $\mathcal{D}=(R, G, w, \lambda)$ as above, we obtain the datum

$$
\mathcal{D}_{H}=\left(R, H,\left.w\right|_{H}, \lambda\right)
$$

for each subgroup $H$ of $G$.

Proposition 6.7. Let $H$ be a subgroup of $G$ and $B: M \times M \rightarrow R[H]$ a $\lambda$-Hermitian form on an $R[H]$-module $M$. Then for each $g \in H$, the following diagrams commute:

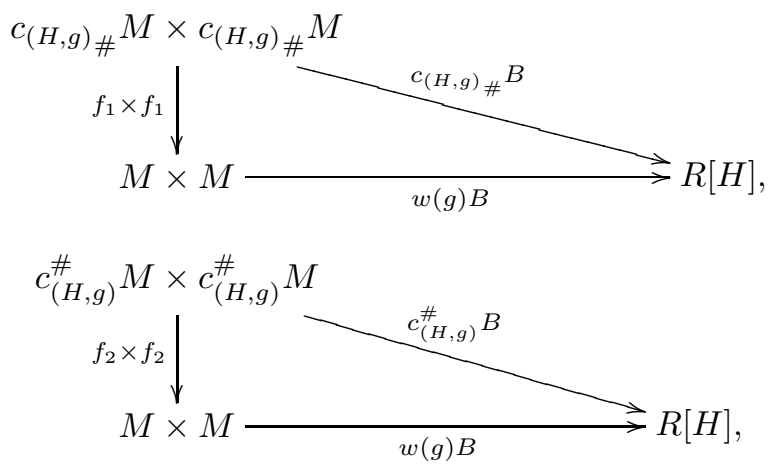

where $f_{1}$ and $f_{2}$ are the canonical isomorphisms (cf. Proposition 3.4). 
Proof. The commutability of the first diagram follows from

$$
\left(c_{(H, g) \#} B\right)(e \otimes x, e \otimes y)=\sum_{h \in H} \varepsilon\left(B\left(x, g^{-1} h^{-1} g y\right)\right) h
$$

and

$$
\begin{aligned}
B\left(f_{1}(e \otimes x), f_{1}(e \otimes y)\right) & =B(g x, g y) \\
& =\sum_{h \in H} \varepsilon\left(B\left(g x, h^{-1} g y\right)\right) h \\
& =w(g) \sum_{h \in H} \varepsilon\left(B\left(x, g^{-1} h^{-1} g y\right)\right) h .
\end{aligned}
$$

The commutability of the second diagram follows from

$$
\left(c_{(H, g)}{ }^{\#} B\right)(x, y)=\sum_{h \in H} \varepsilon\left(B\left(x, g h^{-1} g^{-1} y\right)\right) h
$$

and

$$
\begin{aligned}
B\left(f_{2}(x), f_{2}(y)\right) & =B\left(g^{-1} x, g^{-1} y\right) \\
& =\sum_{h \in H} \varepsilon\left(B\left(g^{-1} x, h^{-1} g^{-1} y\right)\right) h \\
& =w(g) \sum_{h \in H} \varepsilon\left(B\left(x, g^{-1} h^{-1} g y\right)\right) h .
\end{aligned}
$$

Proposition 6.8. For any subgroups $H$ and $K$ of $G$, each $R[H]$-valued $\lambda$-Hermitian form $B: M \times M \rightarrow R[H]$ on an $R[H]$-module $M$ satisfies the $w$-Mackey double coset formula. Namely,

$$
\begin{aligned}
\left(\operatorname{Res}_{K}^{G} \operatorname{Ind}_{H}^{G} B\right) & \circ(\omega \times \omega) \\
& =\sum_{K g H \in K \backslash G / H} w(g)\left(\operatorname{Ind}_{K \cap g H g^{-1}}^{K} c_{\left(H \cap g^{-1} K g, g\right)}{ }_{\#} \operatorname{Res}_{H \cap g^{-1} K g}^{H} B\right),
\end{aligned}
$$

where $\omega$ is the canonical isomorphism (cf. Proposition 3.5). Particularly, in the case $w(G)=\{1\}, B$ satisfies the Mackey double coset formula.

Proof. It suffices to prove that

$$
\begin{aligned}
& \left(\operatorname{Res}_{K}^{G} \operatorname{Ind}_{H}^{G} B\right)(a g \otimes x, b g \otimes y) \\
& \quad=w(g)\left(\operatorname{Ind}_{K \cap g H g^{-1}}^{K} c_{\left(H \cap g^{-1} K g, g\right)}{ }_{\#} \operatorname{Res}_{H \cap g^{-1} K g}^{H} B\right)(a \otimes(e \otimes x), b \otimes(e \otimes y))
\end{aligned}
$$

for any $g \in G, a, b \in K, x, y \in \operatorname{Res}_{H \cap g^{-1} K g}^{H} M$. This equality holds because

$$
\begin{aligned}
\left(\operatorname{Res}_{K}^{G} \operatorname{Ind}_{H}^{G} B\right) & (a g \otimes x, b g \otimes y) \\
& =\sum_{k \in K} w(a g) \delta_{a g H, k^{-1} b g H} \varepsilon\left(B\left(x,(a g)^{-1} k^{-1} b g y\right)\right) k \\
& =w(g) \sum_{k \in K} w(a) \delta_{a g H, k^{-1} b g H} \varepsilon\left(B\left(x, g^{-1}\left(a^{-1} k^{-1} b\right) g y\right)\right) k
\end{aligned}
$$


and

$$
\begin{aligned}
& \left(\operatorname{Ind}_{K \cap g H g^{-1}}^{K} c_{\left(H \cap g^{-1} K g, g\right)} \operatorname{Res}_{H \cap g^{-1} K g}^{H} B\right)(a \otimes(e \otimes x), b \otimes(e \otimes y)) \\
& =\sum_{k \in K} w(a) \delta_{a\left(K \cap g H g^{-1}\right), k^{-1} b\left(K \cap g H g^{-1}\right)} \\
& \quad \cdot\left(c_{\left(H \cap g^{-1} K g, g\right) \#} \operatorname{Res}_{H \cap g^{-1} K g}^{H} B\right)\left(e \otimes x, a^{-1} k^{-1} b(e \otimes y)\right) k \\
& =\sum_{k \in K} w(a) \delta_{a\left(K \cap g H g^{-1}\right), k^{-1} b\left(K \cap g H g^{-1}\right)} B\left(x, g^{-1}\left(a^{-1} k^{-1} b\right) g y\right) k .
\end{aligned}
$$

\section{Positioned quadratic $R[G]$-Modules}

In this paper $\lambda$ stands for either 1 or -1 . Let $w: G \rightarrow\{-1,1\}$ be a group homomorphism. Set

$$
\begin{aligned}
& G^{\lambda}(2)=\{g \in G(2) \mid w(g)=\lambda\}, \\
& G^{-\lambda}(2)=\{g \in G(2) \mid w(g)=-\lambda\} .
\end{aligned}
$$

Clearly we have $g=\lambda \bar{g}$ for $g \in G^{\lambda}(2)$ and $g=-\lambda \bar{g}$ for $g \in G^{-\lambda}(2)$. Let $S$ and $Q$ be conjugation-invariant subsets of $G^{\lambda}(2)$ and $G^{-\lambda}(2)$, respectively. We shall define the Witt group of $\Theta$-positioned quadratic $R[G]$-modules, which is the Wall group (cf. [27]) in the case where $Q, S$ and $\Theta$ are the empty set, and the Bak group (cf. [1], [19]) in the case where $S$ and $\Theta$ are the empty set. The datum

$$
\boldsymbol{A}=(R, G, Q, S, \lambda, w)
$$

is relevant to the group. Define $R$-submodules $A_{s}=A_{s}(G, S ; R), A_{q}=A_{q}(G, S ; R)$ and $\Lambda=\Lambda(G, Q ; R)$ of $A:=R[G]$ as follows:

$$
\begin{aligned}
& A_{s}=R[S]\left(=\langle s \mid s \in S\rangle_{R}\right), \\
& A_{q}=R[G \backslash S]\left(=\langle g \mid g \in G \backslash S\rangle_{R}\right), \\
& \Lambda=\langle x-\lambda \bar{x} \mid x \in A\rangle_{R}+\langle g \mid g \in Q\rangle_{R} .
\end{aligned}
$$

This module $\Lambda$ is called the form parameter generated by $Q$.

Definition 7.1. A map $q: M \rightarrow A_{q} / \Lambda$ is called an $\boldsymbol{A}$-quadratic form (or quadratic form) on $M$ with respect to $B$ if the following conditions (1)-(3) are fulfilled:

(1) $q(g x)=g q(x) \bar{g}$ and $q(r x)=r^{2} q(x)$ in $A_{q} / \Lambda=A /\left(\Lambda+A_{s}\right)$,

(2) $q(x+y)-q(x)-q(y)=B(x, y)$ in $A_{q} / \Lambda=A /\left(\Lambda+A_{s}\right)$,

(3) $\widetilde{q(x)}+\lambda \widetilde{\overline{q(x)}}=B(x, x)$ in $A_{q}=A / A_{s}$,

for all $x, y \in M, g \in G, r \in R$, where $\widetilde{q(x)} \in A_{q}$ is a lifting of $q(x)$.

A triple $(M, B, q)$ consisting of an $R[G]$-module $M$, an $R[G]$-valued $\lambda$-Hermitian form $B$ on $M$ and an $A$-quadratic form $q$ on $M$ with respect to $B$, is called an $A$-quadratic $R[G]$-module (or $\lambda$-quadratic $R[G]$-module).

Let $\Theta$ be a finite $G$-set. A quadruple $(M, B, q, \alpha)$ consisting of an $\boldsymbol{A}$-quadratic $R[G]$-module $(M, B, q)$ and a $G$-map $\alpha: \Theta \rightarrow M$ is called a $\Theta$-positioned $A$ quadratic $R[G]$-module (or $\Theta$-positioned $\lambda$-quadratic $R[G]$-module).

Let $\mathcal{Q}(\boldsymbol{A}, \Theta)$ (or $\mathcal{Q}(R, G, Q, S, \Theta))$ denote the family of all $\Theta$-positioned $\boldsymbol{A}$-quadratic $R[G]$-modules $(M, B, q, \alpha)$ such that $M$ is a stably free $R[G]$-module and $B$ is nonsingular. 
Let $\boldsymbol{M}=(M, B, q, \alpha) \in \mathcal{Q}(\boldsymbol{A}, \Theta)$. The map $\alpha$ is said to be totally isotropic (resp. trivial) if $B(\operatorname{Im}(\alpha), \operatorname{Im}(\alpha))=0$ and $q(\operatorname{Im}(\alpha))=0$ (resp. $\operatorname{Im}(\alpha)=0)$. Set

$$
\begin{aligned}
& \mathcal{Q}(\boldsymbol{A}, \Theta)^{\text {tiso }}=\{(M, B, q, \alpha) \in \mathcal{Q}(\boldsymbol{A}, \Theta) \mid \alpha \text { is totally isotropic }\}, \\
& \mathcal{Q}(\boldsymbol{A}, \Theta)^{\text {triv }}=\{(M, B, q, \alpha) \in \mathcal{Q}(\boldsymbol{A}, \Theta) \mid \alpha \text { is trivial }\} .
\end{aligned}
$$

Let $\mathrm{KQ}_{0}(\boldsymbol{A}, \Theta), \mathrm{KQ}_{0}(\boldsymbol{A}, \Theta)^{\mathrm{t} \text {-iso }}$ and $\mathrm{KQ}_{0}(\boldsymbol{A})$ denote the Grothendieck groups of $\mathcal{Q}(\boldsymbol{A}, \Theta), \mathcal{Q}(\boldsymbol{A}, \Theta)^{\mathrm{t} \text {-iso }}$ and $\mathcal{Q}(\boldsymbol{A}, \Theta)^{\text {triv }}$, respectively, under orthogonal sum.

A stably $R[G]$-free, $R[G]$-direct summand $L$ of $M$ is called a Lagrangian submodule of $\boldsymbol{M}$ if $B(L, L)=0, q(L)=0, L^{\perp}=L$ and $\operatorname{Im}(\alpha) \subset L$, where

$$
L^{\perp}=\{x \in M \mid B(x, y)=0(\forall y \in L)\} .
$$

If $M$ has a Lagrangian submodule, then $M$ is called a null module. The groups defined by

$$
\begin{aligned}
& \mathrm{WQ}_{0}(\boldsymbol{A}, \Theta)=\mathrm{KQ}_{0}(\boldsymbol{A}, \Theta) /\langle\text { null modules in } \mathcal{Q}(\boldsymbol{A}, \Theta)\rangle, \\
& \mathrm{WQ}_{0}(\boldsymbol{A}, \Theta)^{\mathrm{t} \text {-iso }}=\mathrm{KQ}_{0}(\boldsymbol{A}, \Theta)^{\mathrm{t} \text {-iso }} /\left\langle\text { null modules in } \mathcal{Q}(\boldsymbol{A}, \Theta)^{\mathrm{t} \text {-iso }}\right\rangle, \\
& \mathrm{WQ}_{0}(\boldsymbol{A})=\mathrm{KQ}_{0}(\boldsymbol{A}) /\left\langle\text { null modules in } \mathcal{Q}(\boldsymbol{A}, \Theta)^{\mathrm{triv}}\right\rangle
\end{aligned}
$$

are called the Witt groups of $\Theta$-positioned $\boldsymbol{A}$-quadratic $R[G]$-modules. If the context is clear, those Witt groups are also denoted by

$$
\mathrm{WQ}_{0}(R, G, Q, S, \Theta), \quad \mathrm{WQ}_{0}(R, G, Q, S, \Theta)^{\mathrm{t} \text {-iso }}, \quad \mathrm{WQ}_{0}(R, G, Q, S),
$$

respectively.

\section{The SPecial Witt groups}

Let $\boldsymbol{A}=(R, G, Q, S, \lambda, w)$ be as in the previous section, $\Theta$ a finite $G$-set and $\rho^{(2)}: \Theta \rightarrow \mathfrak{P}(S)$ a $G$-map (cf. Section 5). Let $\boldsymbol{M}=(M, B, q, \alpha)$ be a $\Theta$-positioned $A$-quadratic $R[G]$-module, where $\alpha: \Theta \rightarrow M$. The associated map $\nabla_{\boldsymbol{M}}: M \rightarrow$ $\operatorname{Map}(S, R / 2 R)$ is defined by

$$
\nabla_{\boldsymbol{M}}(x)(s)=\varepsilon\left(B\left(\Delta_{\alpha}(s)-x, s x\right)\right)
$$

for $x \in M$ and $s \in S$, where $\Delta_{\alpha}: S \rightarrow M$ is the map defined by (5.1).

If $\boldsymbol{M} \in \mathcal{Q}(\boldsymbol{A}, \Theta)$ satisfies $\nabla_{\boldsymbol{M}}=0$, then we call $\boldsymbol{M}$ a special $\Theta$-positioned $\boldsymbol{A}$ quadratic $R[G]$-module (or a special $\Theta$-positioned $\lambda$-quadratic $R[G]$-module). Set

$$
\begin{aligned}
& \mathcal{S} \mathcal{Q}(\boldsymbol{A}, \Theta)=\left\{\boldsymbol{M} \in \mathcal{Q}(\boldsymbol{A}, \Theta) \mid \nabla_{\boldsymbol{M}}=0\right\}, \\
& \mathcal{S} \mathcal{Q}(\boldsymbol{A}, \Theta)^{\mathrm{t} \text {-iso }}=\left\{\boldsymbol{M} \in \mathcal{Q}(\boldsymbol{A}, \Theta)^{\mathrm{t} \text {-iso }} \mid \nabla_{\boldsymbol{M}}=0\right\}, \\
& \mathcal{S} \mathcal{Q}(\boldsymbol{A}, \Theta)^{\text {triv }}=\left\{\boldsymbol{M} \in \mathcal{Q}(\boldsymbol{A}, \Theta)^{\text {triv }} \mid \nabla_{\boldsymbol{M}}=0\right\}
\end{aligned}
$$

The corresponding Grothendieck groups are denoted by

$$
\mathrm{KSQ}_{0}(\boldsymbol{A}, \Theta), \quad \mathrm{KSQ}_{0}(\boldsymbol{A}, \Theta)^{\mathrm{t} \text {-iso }}, \quad \mathrm{KSQ}_{0}(\boldsymbol{A})
$$

respectively, or by

$$
\mathrm{KSQ}_{0}(R, G, Q, S, \Theta), \mathrm{KSQ}_{0}(R, G, Q, S, \Theta)^{\mathrm{t} \text {-iso }}, \quad \mathrm{KSQ}_{0}(R, G, Q, S)
$$

respectively. Further, define the special Witt groups

$$
\begin{aligned}
& \operatorname{SWQ}_{0}(\boldsymbol{A}, \Theta) \quad\left(=\operatorname{SWQ}_{0}(R, G, Q, S, \Theta)\right), \\
& \operatorname{SWQ}_{0}(\boldsymbol{A}, \Theta)^{\mathrm{t} \text {-iso }} \quad\left(=\operatorname{SWQ}_{0}(R, G, Q, S, \Theta)^{\mathrm{t} \text {-iso }}\right), \\
& \operatorname{SWQ}_{0}(\boldsymbol{A}) \quad\left(=\operatorname{SWQ}_{0}(R, G, Q, S)\right)
\end{aligned}
$$


by

$$
\begin{aligned}
& \operatorname{SWQ}_{0}(\boldsymbol{A}, \Theta)=\mathrm{KSQ}_{0}(\boldsymbol{A}, \Theta) /\langle\text { null modules in } \mathcal{S} \mathcal{Q}(\boldsymbol{A}, \Theta)\rangle \\
& \mathrm{SWQ}_{0}(\boldsymbol{A}, \Theta)^{\mathrm{t} \text {-iso }}=\mathrm{KSQ}_{0}(\boldsymbol{A}, \Theta)^{\mathrm{t} \text {-iso }} /\left\langle\text { null modules in } \mathcal{S} \mathcal{Q}(\boldsymbol{A}, \Theta)^{\mathrm{t} \text {-iso }}\right\rangle, \\
& \mathrm{SWQ}_{0}(\boldsymbol{A})=\mathrm{KSQ}_{0}(\boldsymbol{A}) /\left\langle\text { null modules in } \mathcal{S} \mathcal{Q}(\boldsymbol{A}, \Theta)^{\text {triv }}\right\rangle
\end{aligned}
$$

respectively.

\section{Tensor products of Hermitian modules and quadratic modules}

Let $\boldsymbol{A}=(R, G, Q, S, \lambda, w)$ be as in Section 7, and $\Theta$ a finite $G$-set. Let $\boldsymbol{M}=$ $(M, B, q)$ be an $A$-quadratic $R[G]$-module. By definition, $B$ is a map $M \times M \rightarrow R[G]$ and $q$ is a map $M \rightarrow A_{q} / \Lambda$. We write $G$ as a disjoint union of the form

$$
G=\{e\} \amalg G(2) \amalg C \amalg C^{-1},
$$

where $C$ is a subset of $G$ consisting of elements of order $\geq 3$ and $C^{-1}=\left\{g^{-1} \mid g \in\right.$ $C\}$. Set

$$
\mathcal{Q}(G)=\{e\} \cup\left(G^{\lambda}(2) \backslash S\right) \cup\left(G^{-\lambda}(2) \backslash Q\right) \cup C .
$$

Let $R_{g}$ stand for the $R$-module defined by

$$
R_{g}= \begin{cases}R /(1-\lambda) R & (g=e), \\ R & \left(g \in G^{\lambda}(2)\right), \\ R / 2 R & \left(g \in G^{-\lambda}(2)\right), \\ R & \text { (otherwise), }\end{cases}
$$

for each $g \in G$. Then $q(x), x \in M$, can be regarded as the formal sum

$$
\sum_{g \in \mathcal{Q}(G)} q(x)_{g} g
$$

with $q(x)_{g} \in R_{g}$; namely, $q: M \rightarrow A_{q} / \Lambda$ can be regarded as the map

$$
M \rightarrow \bigoplus_{g \in \mathcal{Q}(G)} R_{g} ; x \longmapsto\left(q(x)_{g}\right) .
$$

We set $q(x)_{g}=\lambda w(g) q(x)_{g^{-1}}$ for $g \in G$ with $g^{-1} \in \mathcal{Q}(G)$. This definition is compatible with the ambiguity of choice of $\mathcal{Q}(G)$, because

$$
\widetilde{q(x)_{g}} g=\lambda w(g) \widetilde{q(x)_{g}} g^{-1} \bmod \Lambda \text {. }
$$

Let $\boldsymbol{M}_{1}=\left(M_{1}, B_{1}, \alpha_{1}\right)$ and $\boldsymbol{M}_{2}=\left(M_{2}, B_{2}, q_{2}, \alpha_{2}\right)$ be objects in $\mathcal{H}(R, G, S, \Theta)$ and $\mathcal{Q}(\boldsymbol{A}, \Theta)$, respectively. We define an object $\boldsymbol{M}_{1} \cdot \boldsymbol{M}_{2}$ in $\mathcal{Q}(\boldsymbol{A}, \Theta)$ as the product of $\boldsymbol{M}_{1}$ and $\boldsymbol{M}_{2}$ as follows. For the sake of convenience, $\boldsymbol{M}=(M, B, q, \alpha)$ stands for $\boldsymbol{M}_{1} \cdot \boldsymbol{M}_{2}$ for a while.

First, $M$ is defined as the $R$-module $M_{1} \otimes_{R} M_{2}$ with the $G$-action: $(g, x \otimes y) \mapsto$ $(g x) \otimes(g y)$, where $g \in G, x \in M_{1}$ and $y \in M_{2}$. Since $M_{1}$ is $R$-free and $M_{2}$ is stably $R[G]$-free, $M$ is stably $R[G]$-free.

Second, $B: M \times M \rightarrow R[G]$ is defined as the $R$-bilinear form such that

$$
B\left(x \otimes y, x^{\prime} \otimes y^{\prime}\right)=\sum_{g \in G} B_{1}\left(x, g^{-1} x^{\prime}\right) \varepsilon\left(B_{2}\left(y, g^{-1} y^{\prime}\right)\right) g .
$$


The equality $B(u, v)=\lambda \overline{B(v, u)}(u, v \in M)$ holds since

$$
\begin{aligned}
B\left(x \otimes y, x^{\prime} \otimes y^{\prime}\right) & =\sum_{g \in G} B_{1}\left(x, g^{-1} x^{\prime}\right) \varepsilon\left(B_{2}\left(y, g^{-1} y^{\prime}\right)\right) g \\
& =\sum_{g \in G} \lambda B_{1}\left(g^{-1} x^{\prime}, x\right) \varepsilon\left(B_{2}\left(g^{-1} y^{\prime}, y\right)\right) g \\
& =\lambda \sum_{g \in G} w(g) B_{1}\left(x^{\prime}, g x\right) \varepsilon\left(B_{2}\left(y^{\prime}, g y\right)\right) g \\
& =\lambda \sum_{g \in G} B_{1}\left(x^{\prime}, g x\right) \varepsilon\left(B_{2}\left(y^{\prime}, g y\right)\right) \overline{g^{-1}} \\
& =\lambda \overline{\sum_{g \in G} B_{1}\left(x^{\prime}, g x\right) \varepsilon\left(B_{2}\left(y^{\prime}, g y\right)\right) g^{-1}} \\
& =\lambda \overline{B\left(x^{\prime} \otimes y^{\prime}, x \otimes y\right)} .
\end{aligned}
$$

The equality $B(a u, b v)=b B(u, v) \bar{a}(a, b \in G, u, v \in M)$ holds because

$$
\begin{aligned}
B\left(a(x \otimes y), b\left(x^{\prime} \otimes y^{\prime}\right)\right) & =\sum_{g \in G} B_{1}\left(a x, g^{-1} b x^{\prime}\right) \varepsilon\left(B_{2}\left(a y, g^{-1} b y^{\prime}\right)\right) g \\
& =b \sum_{h \in G} B_{1}\left(a x, h^{-1} x^{\prime}\right) \varepsilon\left(B_{2}\left(a y, h^{-1} y^{\prime}\right)\right) h \\
& =b \sum_{h \in G} w(a) B_{1}\left(x, a^{-1} h^{-1} x^{\prime}\right) \varepsilon\left(B_{2}\left(y, a^{-1} h^{-1} y^{\prime}\right)\right) h \\
& =b \sum_{h \in G} w(a) B_{1}\left(x,(h a)^{-1} x^{\prime}\right) \varepsilon\left(B_{2}\left(y,(h a)^{-1} y^{\prime}\right)\right) h \\
& =b \sum_{k \in G} w(a) B_{1}\left(x, k^{-1} x^{\prime}\right) \varepsilon\left(B_{2}\left(y, k^{-1} y^{\prime}\right)\right) k a^{-1} \\
& =b B\left(x \otimes y, x^{\prime} \otimes y^{\prime}\right) \bar{a} .
\end{aligned}
$$

Thus, $B$ is an $R[G]$-valued $\lambda$-Hermitian form on $M$. Note that $B_{1}$ and $\varepsilon \circ B_{2}$ are both nonsingular. So, $B_{1} \otimes\left(\varepsilon \circ B_{2}\right)$ is nonsingular, which implies that $B$ is nonsingular.

Third, we describe the definition of $q: M \rightarrow A_{q} / \Lambda$. Let $F\left(M_{1} \times M_{2}\right)$ denote the $R$-free module with basis $\left\{(x, y) \mid x \in M_{1}, y \in M_{2}\right\}$ (although it may not be finitely generated), $T$ the subset of $F\left(M_{1} \times M_{2}\right)$ consisting of all elements of the form

$$
\begin{gathered}
r(x, y)-(r x, y), \quad r(x, y)-(x, r y), \\
\left(x+x^{\prime}, y\right)-(x, y)-\left(x^{\prime}, y\right), \quad \text { or }\left(x, y+y^{\prime}\right)-(x, y)-\left(x, y^{\prime}\right),
\end{gathered}
$$

where $r$ ranges over $R, x$ and $x^{\prime}$ over $M_{1}, y$ and $y^{\prime}$ over $M_{2}$; and let [ ]:F( $M_{1} \times$ $\left.M_{2}\right) \rightarrow M_{1} \otimes M_{2}$ denote the canonical map.

Lemma 9.1. Let $f$ be a map from $F\left(M_{1} \times M_{2}\right)$ to $A_{q} / \Lambda=A /\left(A_{s}+\Lambda\right)$. If the following conditions (1)-(3) are fulfilled for all $r \in R, u, v \in F\left(M_{1} \times M_{2}\right)$ and $t \in T:$

(1) $f(r u)=r^{2} f(u)$,

(2) $f(u+v)=f(u)+f(v)+B([u],[v])$,

(3) $f(t)=0$,

then $f$ factors through $M_{1} \otimes M_{2} \rightarrow A_{q} / \Lambda$. 
The proof is elementary, and we omit it.

Define a map $f: F\left(M_{1} \times M_{2}\right) \rightarrow A_{q} / \Lambda=A /\left(A_{s}+\Lambda\right)$ by

$f\left(\sum_{i} r_{i}\left(x_{i}, y_{i}\right)\right)=\sum_{i} \sum_{g \in \mathcal{Q}(G)} r_{i}^{2} B_{1}\left(x_{i}, g^{-1} x_{i}\right) q_{2}\left(y_{i}\right)_{g} g+\sum_{i<j} r_{i} r_{j} B\left(x_{i} \otimes y_{i}, x_{j} \otimes y_{j}\right)$,

for finitely many distinct $\left(x_{i}, y_{i}\right)$ with $x_{i} \in M_{1}, y_{i} \in M_{2}$, where $r_{i} \in R$.

By definition, we have $f(r u)=r^{2} f(u)$ for all $r \in R$ and $u \in F\left(M_{1} \times M_{2}\right)$.

Note that for $u=\sum_{i} r_{i}\left(x_{i}, y_{i}\right)$ and $v=\sum_{i} r_{i}^{\prime}\left(x_{i}, y_{i}\right)$, we have

$$
\begin{aligned}
f(u+v)= & \sum_{i} \sum_{g \in \mathcal{Q}(G)}\left(r_{i}+r_{i}^{\prime}\right)^{2} B_{1}\left(x_{i}, g^{-1} x_{i}\right) q_{2}\left(y_{i}\right)_{g} g \\
& +\sum_{i<j}\left(r_{i}+r_{i}^{\prime}\right)\left(r_{j}+r_{j}^{\prime}\right) B\left(x_{i} \otimes y_{i}, x_{j} \otimes y_{j}\right) .
\end{aligned}
$$

Thus, we have

$$
\begin{aligned}
& f(u+v)-f(u)-f(v) \\
& \quad=\sum_{i} \sum_{g \in \mathcal{Q}(G)} 2 r_{i} r_{i}^{\prime} B_{1}\left(x_{i}, g^{-1} x_{i}\right) q_{2}\left(y_{i}\right)_{g} g+\sum_{i<j}\left(r_{i} r_{j}^{\prime}+r_{i}^{\prime} r_{j}\right) B\left(x_{i} \otimes y_{i}, x_{j} \otimes y_{j}\right) .
\end{aligned}
$$

On the other hand, in $A_{q} / \Lambda$ we have

$$
\begin{aligned}
B\left(\sum_{i} r_{i} x_{i} \otimes\right. & \left.y_{i}, \sum_{i} r_{i}^{\prime} x_{i} \otimes y_{i}\right)=\sum_{i} r_{i} r_{i}^{\prime} B\left(x_{i} \otimes y_{i}, x_{i} \otimes y_{i}\right) \\
& \quad+\sum_{i<j}\left(r_{i} r_{j}^{\prime} B\left(x_{i} \otimes y_{i}, x_{j} \otimes y_{j}\right)+r_{j} r_{i}^{\prime} B\left(x_{j} \otimes y_{j}, x_{i} \otimes y_{i}\right)\right) \\
= & \sum_{i} r_{i} r_{i}^{\prime} B\left(x_{i} \otimes y_{i}, x_{i} \otimes y_{i}\right) \\
& +\sum_{i<j}\left(r_{i} r_{j}^{\prime} B\left(x_{i} \otimes y_{i}, x_{j} \otimes y_{j}\right)+r_{i}^{\prime} r_{j} B\left(x_{i} \otimes y_{i}, x_{j} \otimes y_{j}\right)\right) .
\end{aligned}
$$

Moreover, in $A /\left(A_{s}+\Lambda\right)$ we have

$$
\begin{aligned}
B\left(x_{i} \otimes y_{i}, x_{i} \otimes y_{i}\right) & =\sum_{g \in G} B_{1}\left(x_{i}, g^{-1} x_{i}\right) \varepsilon\left(B_{2}\left(y_{i}, g^{-1} y_{i}\right)\right) g \\
& =\sum_{g \in \mathcal{Q}(G)} B_{1}\left(x_{i}, g^{-1} x_{i}\right) 2 q_{2}\left(y_{i}\right)_{g} g .
\end{aligned}
$$

Thus we obtain $f(u+v)-f(u)-f(v)=B([u],[v])$ in $A_{s} / \Lambda$.

It is clear that $f(t)=0$ for all $t \in T$.

Since the conditions (1)-(3) in Lemma 9.1 are satisfied, we obtain the map $q: M \rightarrow A_{q} / \Lambda$ by $q([u])=f(u)$ for $u \in F\left(M_{1} \times M_{2}\right)$. Immediately we have $q(r[u])=r^{2} q([u])$ and $q([u+v])-q([u])-q([v])=B([u],[v])$ for $r \in R$ and $u$, 
$v \in F\left(M_{1} \times M_{2}\right)$. For $g \in G$ and $u=(x, y)$, we have

$$
\begin{aligned}
q(g[u]) & =f(g x, g y) \\
& =\sum_{h \in \mathcal{Q}(G)} B_{1}\left(g x, h^{-1} g x\right) q_{2}(g y)_{h} h \\
& =\sum_{h \in \mathcal{Q}(G)} w(g) B_{1}\left(x, g^{-1} h^{-1} g x\right) q_{2}(y)_{g^{-1} h g} h \\
& =\sum_{h \in \mathcal{Q}(G)} w(g) B_{1}\left(x, k^{-1} x\right) q_{2}(y)_{k} g k g^{-1} \\
& =g \sum_{h \in \mathcal{Q}(G)} B_{1}\left(x, k^{-1} x\right) q_{2}(y)_{k} k \bar{g} \\
& =g f(x \otimes y) \bar{g} \\
& =g q([u]) \bar{g}
\end{aligned}
$$

where $k=g^{-1} h g$. Thus, $q(g z)=g q(z) \bar{g}$ for all $g \in G$ and $z \in M$.

Next we check the property (3) in Definition 7.1. For $u=(x, y)$ we have

$$
\begin{aligned}
\widetilde{q([u])}+\overline{\overline{q([u])}} & \left.=\sum_{g \in \mathcal{Q}(G)} B_{1}\left(x, g^{-1} x\right) \widetilde{\left(q_{2}(y)_{g}\right.} g+\widehat{\lambda q_{2}(y)_{g}} \bar{g}\right) \\
& =\sum_{g \in G} B_{1}\left(x, g^{-1} x\right) B_{2}(y, y)_{g} g \\
& =B([u],[u]) \quad \text { in } A_{q}=A / A_{s},
\end{aligned}
$$

which shows that $\widetilde{q(z)}+\lambda \overline{\overline{q(z)}}=B(z, z)$ for all $z \in M$.

Putting all together, we see that the current triple $(M, B, q)$ is an $\boldsymbol{A}$-quadratic $R[G]$-module.

Defining $\alpha: \Theta \rightarrow M$ by $\alpha(t)=\alpha_{1}(t) \otimes \alpha_{2}(t)$ for $t \in \Theta$, we establish $\boldsymbol{M}_{1} \cdot \boldsymbol{M}_{2}$ $(=\boldsymbol{M}=(M, B, q, \alpha))$ from $\boldsymbol{M}_{1}=\left(M_{1}, B_{1}, \alpha_{1}\right)$ and $\boldsymbol{M}_{2}=\left(M_{2}, B_{2}, q_{2}, \alpha_{2}\right)$.

Theorem 9.2. Let $\boldsymbol{A}=(R, G, Q, S, \lambda, w)$ and $\Theta$ be as above. Then

$$
\mathrm{WQ}_{0}(\boldsymbol{A}, \Theta), \quad \mathrm{WQ}_{0}(\boldsymbol{A}, \Theta)^{\mathrm{t} \text {-iso }} \text { and } \mathrm{WQ}_{0}(\boldsymbol{A})
$$

are modules over $\mathrm{GW}_{0}(R, G, S, \Theta)$, and $\mathrm{WQ}_{0}(\boldsymbol{A})$ is one over $\mathrm{GW}_{0}(R, G, S)$ by the pairing

$$
\left(\boldsymbol{M}_{1}, \boldsymbol{M}_{2}\right) \longmapsto \boldsymbol{M}_{1} \cdot \boldsymbol{M}_{2}
$$

\section{TENSOR PRODUCTS AND $\nabla$-INVARIANTS}

In this section we invoke that $R$ is square identical. Let $Q, S, w, \lambda$ and $\Theta$ be as in Section 7, and let $\rho^{(2)}: \Theta \rightarrow \mathfrak{P}(S)$ be a $G$-map such that for every $s \in S$, there exists exactly one $t \in \Theta$ with $\rho^{(2)}(t)=s$. Hence, by Proposition 5.4 $\operatorname{SGW}_{0}(R, G, S, \Theta)$ is a commutative ring with unit.

Proposition 10.1. Let $\boldsymbol{M}_{1}=\left(M_{1}, B_{1}, \alpha_{1}\right)$ and $\boldsymbol{M}_{2}=\left(M_{2}, B_{2}, q_{2}, \alpha_{2}\right)$ be objects in $\mathcal{S H}(R, G, S, \Theta)$ and $\mathcal{S} \mathcal{Q}(\boldsymbol{A}, \Theta)$, respectively. Then $\boldsymbol{M}=\boldsymbol{M}_{1} \cdot \boldsymbol{M}_{2}=(M, B, q, \alpha)$ defined in the previous section lies in $\mathcal{S} Q(A, \Theta)$. 
Proof. It was already shown that $\boldsymbol{M}=\boldsymbol{M}_{1} \cdot \boldsymbol{M}_{2}$ belongs to $\mathcal{Q}(\boldsymbol{A}, \Theta)$. Therefore, it suffices to show that $\nabla_{\boldsymbol{M}}=0$. By definition, we have

$$
\begin{aligned}
\nabla_{\boldsymbol{M}}(x \otimes y)(s)= & \varepsilon\left(B\left(\Delta_{\alpha}(s)-x \otimes y, s(x \otimes y)\right)\right) \\
= & \varepsilon\left(B\left(\Delta_{\alpha_{1}}(s) \otimes \Delta_{\alpha_{2}}(s)-x \otimes y, s x \otimes s y\right)\right) \\
= & \varepsilon\left(B\left(\Delta_{\alpha_{1}}(s) \otimes \Delta_{\alpha_{2}}(s), s x \otimes s y\right)\right)-\varepsilon(B(x \otimes y, s x \otimes s y)) \\
= & B_{1}\left(\Delta_{\alpha_{1}}(s), s x\right) \varepsilon\left(B_{2}\left(\Delta_{\alpha_{2}}(s), s y\right)\right)-B_{1}(x, s x) \varepsilon\left(B_{2}(y, s y)\right) \\
= & B_{1}\left(\Delta_{\alpha_{2}}(s)-x, s x\right) \varepsilon\left(B_{2}\left(\Delta_{\alpha_{2}}(s), s y\right)\right) \\
& +B_{1}(x, s x) \varepsilon\left(B_{2}\left(\Delta_{\alpha_{2}}(s)-y, s y\right)\right) \\
= & \nabla_{\boldsymbol{M}_{1}}(x)(s) \varepsilon\left(B_{2}\left(\Delta_{\alpha_{2}}(s), s y\right)\right)+B_{1}(x, s x) \nabla_{\boldsymbol{M}_{2}}(y)(s) \\
= & 0 \quad \text { in } R / 2 R
\end{aligned}
$$

for $x \in M_{1}, y \in M_{2}$, and $s \in S$. By using Proposition $5.2(1)$, we have $\nabla_{M}=0$.

The next theorem follows.

Theorem 10.2. Let $\boldsymbol{A}=(R, G, Q, S, \lambda, w)$ and $\Theta$ be as above. Then

$$
\mathrm{SWQ}_{0}(\boldsymbol{A}, \Theta), \quad \mathrm{SWQ}_{0}(\boldsymbol{A}, \Theta)^{\mathrm{t} \text {-iso }} \text { and } \mathrm{SWQ}_{0}(\boldsymbol{A})
$$

are modules over $\mathrm{SGW}_{0}(R, G, S, \Theta)$.

\section{The Mackey and Green structures of GW and SGW}

Let $S$ be a conjugation-invariant subset of $G(2)$, and set

$$
S_{H}=H \cap S
$$

for each $H \in \mathcal{S}(G)$. Let $Z^{(0)}$ be a finite $G$-set and let $\mathfrak{P}\left(Z^{(0)}\right)$ stand for the set of all subsets of $Z^{(0)}$. Let $\mathcal{S}(G) \rightarrow \mathfrak{P}\left(Z^{(0)}\right) ; H \mapsto Z_{H}^{(0)}$, be an intersection-preserving $G$-map (see (3.1)), where $\mathcal{S}(G)$ is the set of all subgroups of $G$ on which $G$ acts by conjugation.

Define $\Theta_{H}$ by

$$
\Theta_{H}=S_{H} \amalg Z_{H}^{(0)} .
$$

It immediately follows that the map $H \mapsto \Theta_{H}$ is intersection preserving. Define $\rho_{H}^{(2)}: \Theta_{H} \rightarrow \mathfrak{P}\left(S_{H}\right)$ by

$$
\rho_{H}^{(2)}(t)= \begin{cases}\{t\} & \left(t \in S_{H}\right), \\ \emptyset & \left(t \in Z_{H}^{(0)}\right) .\end{cases}
$$

Then, obviously, for each $s \in S_{H}$, there exists exactly one $t \in \Theta_{H}$ with $s \in \rho_{H}^{(2)}(t)$. In this case, $\mathrm{GW}_{0}\left(R, H, \Theta_{H}\right)$ is a commutative ring with unit for each subgroup $H$ of $G$, and so is $\mathrm{SGW}_{0}\left(R, H, S_{H}, \Theta_{H}\right)$ if $R$ is square identical.

Now let $\varphi: H \rightarrow K$ be a morphism in $\mathcal{G}$, namely one of an inclusion map, a conjugation map, or a composition of such maps. Then we have the associated $\varphi$ equivariant map $\psi: \Theta_{H} \rightarrow \Theta_{K}$. Actually, if $\varphi$ is the inclusion map $j_{H, K}: H \rightarrow K$, then $S_{H} \subset S_{K}$ and $Z_{H}^{(0)} \subset Z_{K}^{(0)}$, and therefore the associated $\psi: \Theta_{H} \rightarrow \Theta_{K}$ is the inclusion map; if $\varphi$ is the conjugation map $c_{(H, g)}: H \rightarrow g \mathrm{Hg}^{-1}$, then the associated $\psi: \Theta_{H} \rightarrow \Theta_{g H g^{-1}}=g \Theta_{H}$ is the left translation $\ell_{\left(\Theta_{H}, g\right)}$ by $g$. Since the $G$-action on $S$ is given by conjugation, $\left.\ell_{\left(\Theta_{H}, g\right)}\right|_{S_{H}}$ is the conjugation $\left.c_{(H, g)}\right|_{S_{H}}$ by $g$. Thus, there are canonical correspondences

$$
\operatorname{GW}_{0}\left(R, H, \Theta_{H}\right) \rightarrow \mathrm{GW}_{0}\left(R, K, \Theta_{K}\right) ;[M, B, \alpha] \mapsto\left[\varphi_{\#} M, \varphi_{\#} B, \psi_{\#} \alpha\right]
$$


and

$$
\mathrm{GW}_{0}\left(R, K, \Theta_{K}\right) \rightarrow \mathrm{GW}_{0}\left(R, H, \Theta_{H}\right) ;[N, B, \alpha] \mapsto\left[\varphi^{\#} N, \varphi^{\#} B, \psi^{\#} \alpha\right] .
$$

Lemma 11.1. $\nabla_{\varphi_{\#} M}=0$ for any morphism $\varphi: H \rightarrow K$ in $\mathcal{G}$ and any object $\boldsymbol{M}=(M, B, \alpha)$ in $\mathcal{S} \mathcal{H}\left(R, H, \Theta_{H}\right)$.

Proof. For the proof, we may suppose that $\varphi=j_{H, K}$ or $c_{(H, g)}$. For any $z=k \otimes_{\varphi} x$ $\in \varphi_{\#} M$ with $k \in K, x \in M$ and $s \in S_{K}$, we have

$$
\begin{aligned}
& \nabla_{\boldsymbol{\varphi}_{\#} \boldsymbol{M}}\left(k \otimes_{\varphi} x\right)(s)=\varphi_{\#} B\left(\Delta_{\psi_{\#} \alpha}(s)-k \otimes_{\varphi} x, s\left(k \otimes_{\varphi} x\right)\right) \\
& \quad=\varphi_{\#} B\left(\Delta_{\psi_{\#} \alpha}(s), s\left(k \otimes_{\varphi} x\right)\right)-\varphi_{\#} B\left(k \otimes_{\varphi} x, s\left(k \otimes_{\varphi} x\right)\right) \quad \text { in } R / 2 R .
\end{aligned}
$$

By definition, we have

$$
\begin{array}{rl}
\varphi_{\#} & B\left(\Delta_{\psi_{\#} \alpha}(s), s\left(k \otimes_{\varphi} x\right)\right)=\varphi_{\#} B\left(\Delta_{\psi_{\#} \alpha}(s), k \otimes_{\varphi} x\right) \\
= & \varphi_{\#} B\left(\psi_{\#} \alpha(s), k \otimes_{\varphi} x\right) \\
= & \sum_{\left[a, s^{\prime}\right] \in K \times_{H, \varphi} \Theta_{H}}\left\{\varphi_{\#} B\left(a \otimes_{\varphi} \alpha\left(s^{\prime}\right), k \otimes_{\varphi} x\right) \mid s^{\prime} \in S_{H}, a \varphi\left(s^{\prime}\right) a^{-1}=s\right\} \\
= & \sum_{\left[a, s^{\prime}\right] \in K \times_{H, \varphi} \Theta_{H}}\left\{\delta_{a \varphi(H), k \varphi(H)} B\left(\alpha\left(s^{\prime}\right), \varphi^{-1}\left(a^{-1} k\right) x\right) \mid s^{\prime} \in S_{H}, \varphi\left(s^{\prime}\right)=a^{-1} s a\right\} \\
= & \sum_{\left[a, s^{\prime}\right] \in K \times_{H, \varphi} \Theta_{H}}\left\{\delta_{\varphi(H), a^{-1} k \varphi(H)} B\left(\alpha\left(s^{\prime}\right), \varphi^{-1}\left(a^{-1} k\right) x\right) \mid s^{\prime} \in S_{H}, \varphi\left(s^{\prime}\right)=a^{-1} s a\right\} \\
= & \sum_{\left[k, s^{\prime \prime}\right] \in K \times_{H, \varphi} \Theta_{H}}\left\{B\left(\alpha\left(s^{\prime \prime}\right), x\right) \mid s^{\prime \prime} \in S_{H}, \varphi\left(s^{\prime \prime}\right)=k^{-1} s k\right\} \\
= & \sum_{\left[k, s^{\prime \prime}\right] \in K \times_{H, \varphi} \Theta_{H}}\left\{B\left(\alpha\left(s^{\prime \prime}\right), s^{\prime \prime} x\right) \mid s^{\prime \prime} \in S_{H}, \varphi\left(s^{\prime \prime}\right)=k^{-1} s k\right\} \\
= & \sum_{\left[k, s^{\prime \prime}\right] \in K \times_{H, \varphi} \Theta_{H}}\left\{B\left(x, s^{\prime \prime} x\right) \mid s^{\prime \prime} \in S_{H}, \varphi\left(s^{\prime \prime}\right)=k^{-1} s k\right\} \\
= & \begin{cases}\left.B\left(x, \varphi^{-1}\left(k^{-1} s k\right) x\right) \quad \text { (if } k^{-1} s k \in \varphi(H)\right), \\
0 & \text { (otherwise). }\end{cases}
\end{array}
$$

On the other hand,

$$
\begin{aligned}
\varphi_{\#} B\left(\left(k \otimes_{\varphi} x\right), s\left(k \otimes_{\varphi} x\right)\right) & =\varphi_{\#} B\left(k \otimes_{\varphi} x, s k \otimes_{\varphi} x\right) \\
& =\delta_{k \varphi(H), s k \varphi(H)} B\left(x, \varphi^{-1}\left(k^{-1} s k\right) x\right) \\
& = \begin{cases}B\left(x, \varphi^{-1}\left(k^{-1} s k\right) x\right) & \text { (if } \left.k^{-1} s k \in \varphi(H)\right), \\
0 & \text { (otherwise) } .\end{cases}
\end{aligned}
$$

This gives us $\nabla_{\boldsymbol{\varphi}_{\#} \boldsymbol{M}}(z)(s)=0$ for all $z \in \varphi_{\#} M$ and $s \in \Theta_{K}$.

Proposition 11.2. Let $S_{H}, Z_{H}$ and $\Theta_{H}$ be as above. Then, the Grothendieck-Witt ring functor $H \mapsto \mathrm{GW}_{0}\left(R, H, \Theta_{H}\right), H \in \mathcal{S}(G)$, is a Mackey functor, and so is the special Grothendieck-Witt ring functor $\mathrm{SGW}_{0}\left(R, H, S_{H}, \Theta_{H}\right), H \in \mathcal{S}(G)$.

Proof. This follows from Propositions 3.2, 3.4 3.5, 4.3, 4.5 and 4.6. and Lemma 11.1.

Theorem 11.3. Let $S_{H}, Z_{H}$ and $\Theta_{H}$ be as above. Then, the Grothendieck-Witt ring functor $H \mapsto \mathrm{GW}_{0}\left(R, H, \Theta_{H}\right), H \in \mathcal{S}(G)$, is a Green functor, and the special 
Grothendieck-Witt ring functor $H \mapsto \mathrm{SGW}_{0}\left(R, H, S_{H}, \Theta_{H}\right), H \in \mathcal{S}(G)$, is a Green functor, possibly without unit. If $R$ is square identical, then the functor $H \mapsto$ $\mathrm{SGW}_{0}\left(R, H, S_{H}, \Theta_{H}\right), H \in \mathcal{S}(G)$, is a Green functor.

Proof. The theorem follows from Propositions 3.1 3.3 4.2, 4.4, 4.10 and 5.4.

Theorem 11.4. The special Grothendieck-Witt group functor

$$
H \mapsto \operatorname{SGW}_{0}\left(R, H, S_{H}\right)
$$

is a module over the Grothendieck-Witt ring functor $H \rightarrow \mathrm{GW}_{0}\left(R, H, S_{H}\right)$.

Proof. By Proposition [5.5. $\mathrm{SGW}_{0}\left(R, H, S_{H}\right)$ is a module over $\mathrm{GW}_{0}(R, H)$. The required properties for a Frobenius pairing follow from Propositions [3.1 3.3, 4.2 and 4.4 .

$$
\text { 12. The Pairing } \mathrm{SGW}_{0} \times \mathrm{SWQ}_{0} \rightarrow \mathrm{SWQ}_{0}
$$

Let $S \subset G(2), S_{H}, Z_{H}^{(0)}, \Theta_{H}, \rho_{H}^{(2)}$ be as in Section 11, where $H \in \mathcal{S}(G)$. Let $w: G \rightarrow\{-1,1\}$ be a homomorphism and let $\lambda$ stand for either 1 or -1 . In the current section we invoke

$$
S \subset G^{\lambda}(2) \text {. }
$$

Let $Q$ be a conjugation-invariant subset of $G^{-\lambda}(2)$. We set $Q_{H}=H \cap Q, A_{H}=$ $R[H]$, and $\boldsymbol{A}_{H}=\left(R, H, Q_{H}, S_{H}, \lambda,\left.w\right|_{H}\right)$ for $H \in \mathcal{S}(G)$.

Let $\varphi: H \rightarrow K$, where $H, K \in \mathcal{S}(G)$, be a monomorphism such that $\left.w\right|_{K} \circ \varphi=$ $\left.w\right|_{H}, \varphi\left(Q_{H}\right) \subset Q_{K}$, and $\varphi\left(S_{H}\right) \subset S_{K}$.

Let $\boldsymbol{N}=(N, B, q)$ be an $\boldsymbol{A}_{K}$-quadratic $R[K]$-module. We can write $q(x)$ as $\sum_{g \in \mathcal{Q}(K)} q(x)_{g} g$, where $\mathcal{Q}(K)=K \cap \mathcal{Q}(G)$ and $q(x)_{g} \in R_{g}$. We define $\varphi^{\#} q$ : $\varphi^{\#} M \rightarrow\left(A_{H}\right)_{q} / \Lambda_{H}=R[H] /\left(R\left[S_{H}\right]+\Lambda_{H}\right)$ by

$$
\varphi^{\#} q(x)=\sum_{h \in \mathcal{Q}(H)} q(x)_{\varphi(h)} h
$$

for $x \in \varphi^{\#} M$, where $\Lambda_{H}$ is the smallest form parameter of $R[H]$ including $Q_{H}$.

Lemma 12.1. The $\varphi^{\#} q$ above is an $\boldsymbol{A}_{H}$-quadratic form on $\varphi^{\#} N$ with respect to $\varphi^{\#} B$.

Proof. The proof is straightforward, as follows: For $g \in H$ and $x \in \varphi^{\#} N$, we have

$$
\begin{aligned}
\varphi^{\#} q(g x) & =\sum_{h \in \mathcal{Q}(H)} q(g x)_{\varphi(h)} h \\
& =\sum_{h \in \mathcal{Q}(H)} q(\varphi(g) x)_{\varphi(h)} h \\
& =\sum_{h \in \mathcal{Q}(H)} w(\varphi(g)) q(x)_{\varphi(g)^{-1} \varphi(h) \varphi(g)} h \\
& =g\left(\sum_{h \in \mathcal{Q}(H)} q(x)_{\varphi\left(g^{-1} h g\right)} g^{-1} h g\right) \bar{g} \\
& =g \varphi^{\#} q(x) \bar{g} .
\end{aligned}
$$


For $x, y \in \varphi^{\#} N$, we have

$$
\begin{aligned}
\varphi^{\#} q(x+y)-\varphi^{\#} q(x)-\varphi^{\#} q(x) & =\sum_{h \in \mathcal{Q}(H)}\left(q(x+y)_{\varphi(h)}-q(x)_{\varphi(h)}-q(y)_{\varphi(h)}\right) h \\
& =\sum_{h \in H} B(x, y)_{\varphi(h)} h \\
& =\sum_{h \in H} \varepsilon\left(B\left(x, \varphi(h)^{-1} y\right)\right) h \\
& =\varphi^{\#} B(x, y)
\end{aligned}
$$

in $A_{H} /\left(\Lambda_{H}+\left(A_{H}\right)_{s}\right)$.

For $x \in \varphi^{\#} N$, we have

$$
\begin{aligned}
\widetilde{\varphi^{\#} q(x)}+\overline{\widetilde{\varphi^{\#} q(x)}} & =\sum_{h \in \mathcal{Q}(H)}\left(\widetilde{q(x)_{\varphi(h)}} h+\lambda q \widetilde{(x)_{\varphi(h)}} \bar{h}\right) \\
& =\sum_{h \in \mathcal{Q}(H)}\left(\widetilde{q(x)_{\varphi(h)}} h+\lambda w(h) q \widetilde{(x)_{\varphi(h)}} h^{-1}\right) \\
& =\sum_{h \in \mathcal{Q}(H)}\left(\widetilde{q(x)_{\varphi(h)}} h+q\left(\widetilde{x)_{\varphi(h)^{-1}}} h^{-1}\right)\right. \\
& =\varphi^{\#} B(x, x) \text { in } A_{H} /\left(A_{H}\right)_{s} .
\end{aligned}
$$

Proposition 12.2. Let $\varphi: H \rightarrow K, \boldsymbol{A}_{H}$ and $\boldsymbol{A}_{K}$ be as above, and let $\boldsymbol{M}_{1}=$ $\left(M_{1}, B_{1}\right)$ and $\boldsymbol{M}_{2}=\left(M_{2}, B_{2}, q_{2}\right)$ be a Hermitian $R[K]$-module and an $\boldsymbol{A}_{K}$-quadratic module, respectively. Then $\left(\varphi^{\#} \boldsymbol{M}_{1}\right) \cdot\left(\varphi^{\#} \boldsymbol{M}_{2}\right)=\varphi^{\#}\left(\boldsymbol{M}_{1} \cdot \boldsymbol{M}_{2}\right)$.

Proof. Let $x, x^{\prime} \in M_{1}$ and $y, y^{\prime} \in M_{2}$. Then

$$
\begin{aligned}
B_{\varphi \# \boldsymbol{M}_{1} \cdot \varphi^{\#} \boldsymbol{M}_{2}}\left(x \otimes y, x^{\prime} \otimes y^{\prime}\right) & =\sum_{h \in H} B_{1}\left(x, \varphi(h)^{-1} x^{\prime}\right) \varepsilon\left(B_{2}\left(y, \varphi(h)^{-1} y^{\prime}\right)\right) h \\
& =B_{\varphi \#\left(\boldsymbol{M}_{1} \cdot \boldsymbol{M}_{2}\right)}\left(x \otimes y, x^{\prime} \otimes y^{\prime}\right) .
\end{aligned}
$$

In addition,

$$
\begin{aligned}
q_{\varphi \# \boldsymbol{M}_{1} \cdot \varphi^{\#} \boldsymbol{M}_{2}}(x \otimes y) & =\sum_{h \in \mathcal{Q}(H)} B_{1}\left(x, \varphi(h)^{-1} x\right) q_{2}(y)_{\varphi(h)} h \\
& =q_{\varphi \#\left(\boldsymbol{M}_{1} \cdot \boldsymbol{M}_{2}\right)}(x \otimes y) .
\end{aligned}
$$

We have established the proposition.

Now let $\boldsymbol{M}=(M, B, q)$ be an $\boldsymbol{A}_{H}$-quadratic $R[H]$-module such that $M$ is stably $R[H]$-free and $B$ is nonsingular. Let $\left\{g_{1}, \ldots, g_{\ell}\right\}$ be a complete set of representatives of $K / \varphi(H)$, where $g_{i}$ are elements in $K$. We define $\varphi_{\#} q: \varphi_{\#} M \rightarrow\left(A_{K}\right)_{q} / \Lambda=$ $A_{K} /\left(\Lambda_{K}+\left(A_{K}\right)_{s}\right)$ by

$$
\varphi_{\#} q\left(\sum_{i=1}^{\ell} g_{i} \otimes_{\varphi} x_{i}\right)=\sum_{i=1}^{\ell} g_{i} \varphi\left(q\left(x_{i}\right)\right) \overline{g_{i}}+\sum_{1 \leq i<j \leq \ell} g_{j} \varphi\left(B\left(x_{i}, x_{j}\right)\right) \overline{g_{i}} .
$$


Lemma 12.3. The $\varphi_{\#} q$ above is a quadratic form on $\varphi_{\#} M$ with respect to $\varphi_{\#} B$. Namely, the following hold:

(1) $\varphi_{\#} q(r u)=r^{2} \varphi_{\#} q(u)$,

(2) $\varphi_{\#} q(u+v)-\varphi_{\#} q(u)-\varphi_{\#} q(v)=\varphi_{\#} B(u, v)$,

(3) $\widetilde{\varphi_{\#} q(u)}+\lambda \widetilde{\varphi_{\#} q(u)}=\varphi_{\#} B(u, u)$ in $A_{K} /\left(A_{K}\right)_{s}$,

(4) $\varphi_{\#} q(k u)=k \varphi_{\#} q(u) \bar{k}$,

for all $r \in R, u, v \in \varphi_{\#} M, k \in K$.

Proof. The equality (1) holds clearly.

The proof of (2) runs as follows:

$$
\begin{aligned}
\varphi_{\#} q\left(\sum_{i} g_{i} \otimes_{\varphi} x_{i}+\right. & \left.\sum_{i} g_{i} \otimes_{\varphi} y_{i}\right)-\varphi_{\#} q\left(\sum_{i} g_{i} \otimes_{\varphi} x_{i}\right)-\varphi_{\#} q\left(\sum_{i} g_{i} \otimes_{\varphi} y_{i}\right) \\
= & \sum_{i=1}^{\ell} g_{i}\left(\varphi\left(q\left(x_{i}+y_{i}\right)\right)-\varphi\left(q\left(x_{i}\right)\right)-\varphi\left(q\left(y_{i}\right)\right)\right) \overline{g_{i}} \\
& +\sum_{1 \leq i<j \leq \ell} g_{j} \varphi\left(B\left(x_{i}+y_{i}, x_{j}+y_{j}\right)-B\left(x_{i}, x_{j}\right)-B\left(y_{i}, y_{j}\right)\right) \overline{g_{i}} \\
= & \sum_{i=1}^{\ell} g_{i} \varphi\left(B\left(x_{i}, y_{i}\right)\right) \overline{g_{i}}+\sum_{1 \leq i \neq j \leq \ell} g_{j} \varphi\left(B\left(x_{i}, y_{j}\right)\right) \overline{g_{i}} \\
= & \varphi_{\#} B\left(\sum_{i=1}^{\ell} g_{i} \otimes_{\varphi} x_{i}, \sum_{j=1}^{\ell} g_{j} \otimes_{\varphi} y_{j}\right) .
\end{aligned}
$$

The equality (3) holds because

$$
\begin{aligned}
\left.\varphi_{\#} \widetilde{q\left(g_{i} \otimes_{\varphi}\right.} x\right) & +\overline{\varphi_{\#} \widetilde{q\left(g_{i} \otimes_{\varphi} x\right)}} \\
& =g_{i} \varphi(\widetilde{(x)}) \overline{g_{i}}+\lambda g_{i} \varphi(\overline{\widetilde{q(x)}}) \overline{g_{i}} \\
& =g_{i} \varphi(B(x, x)) \overline{g_{i}} \\
& =\varphi_{\#} B\left(g_{i} \otimes_{\varphi} x, g_{i} \otimes_{\varphi} x\right) .
\end{aligned}
$$

For $k \in K$, we can write $k g_{i}$ in the form $g_{\sigma(i)} \varphi\left(h_{i}\right)$ with $h_{i} \in H$. Then

$$
\begin{aligned}
\varphi_{\#} q\left(k\left(g_{i} \otimes_{\varphi} x\right)\right) & =\varphi_{\#} q\left(g_{\sigma(i)} \otimes_{\varphi} h_{i} x\right) \\
& =g_{\sigma(i)} \varphi\left(q\left(h_{i} x\right)\right) \overline{g_{\sigma(i)}} \\
& =g_{\sigma(i)} \varphi\left(h_{i}\right) \varphi(q(x)) \overline{g_{\sigma(i)} \varphi\left(h_{i}\right)} \\
& =k g_{i} \varphi(q(x)) \overline{g_{i}} \bar{k} \\
& =k \varphi_{\#} q\left(g_{i} \otimes_{\varphi} x\right) \bar{k} .
\end{aligned}
$$

The equation (4) follows from this and (2) above. 
Proposition 12.4. Let $H$ be a subgroup of $G, q: M \rightarrow\left(A_{H}\right)_{q} / \Lambda_{H}$ an $\boldsymbol{A}_{H^{-}}$ quadratic form on $M$, and $g$ an element of $G$. Then the diagram

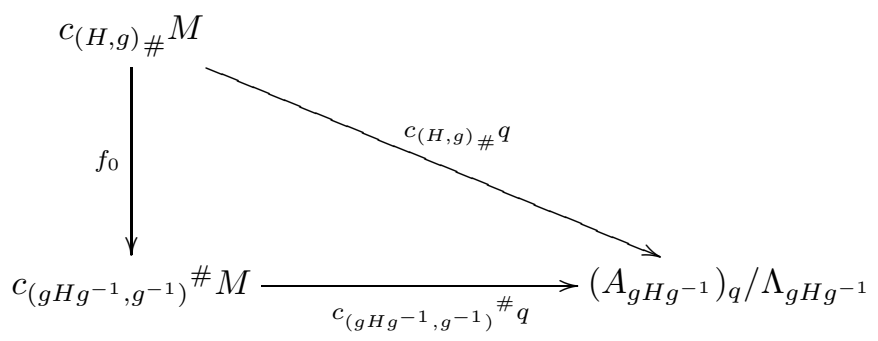

commutes, where $f_{0}$ is the canonical $R\left[g \mathrm{Hg}^{-1}\right]$-isomorphism (cf. Proposition 3.2).

The proof of the proposition is straightforward.

Proposition 12.5. Let $H$ be a subgroup of $G$ and $q: M \rightarrow\left(A_{H}\right)_{q} / \Lambda_{H}$ an $\boldsymbol{A}_{H^{-}}$ quadratic form on $M$. Then for each $g \in H$, the following diagrams commute:
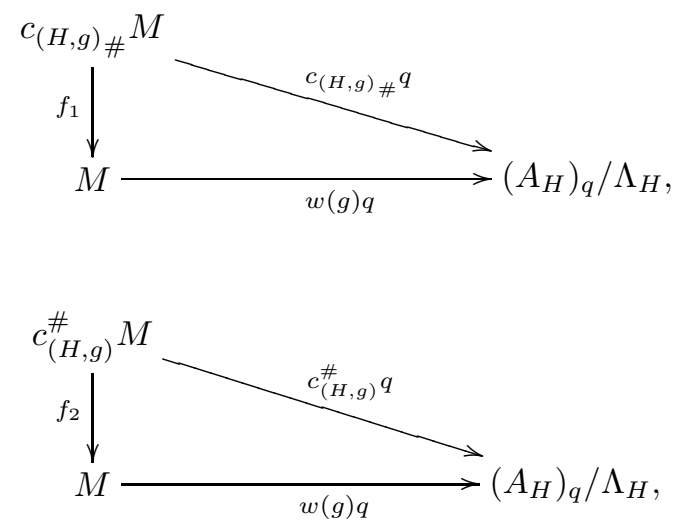

where $f_{1}$ and $f_{2}$ are the canonical isomorphisms (cf. Proposition 3.4).

The proposition follows straightforwardly from the definition.

Proposition 12.6. For any subgroups $H$ and $K$ of $G$, each $A_{H}$-quadratic form $q: M \rightarrow\left(A_{H}\right)_{q} / \Lambda_{H}$ satisfies the $w$-Mackey double coset formula. Namely,

$$
\left(\operatorname{Res}_{K}^{G} \operatorname{Ind}_{H}^{G} q\right) \circ \omega=\sum_{K g H \in K \backslash G / H} w(g) \operatorname{Ind}_{K \cap g H g^{-1}}^{K} c_{\left(H \cap g^{-1} K g, g\right)} \operatorname{Res}_{H \cap g^{-1} K g}^{H} q,
$$

where $\omega$ is the canonical isomorphism (cf. Proposition 3.5). Particularly, in the case $w(G)=\{1\}$, q satisfies the Mackey double coset formula.

Proof. It suffices to prove that

$\left(\operatorname{Res}_{K}^{G} \operatorname{Ind}_{H}^{G} q\right)(a g \otimes x)=w(g)\left(\operatorname{Ind}_{K \cap g H g^{-1}}^{K} c_{\left(H \cap g^{-1} K g, g\right)}{ }_{\#} \operatorname{Res}_{H \cap g^{-1} K g}^{H} q\right)(a \otimes(e \otimes x))$ 
for any $g \in G, a \in K, x \in \operatorname{Res}_{H \cap g^{-1} K g}^{H} M$. This is valid because

$$
\begin{aligned}
\left(\operatorname{Res}_{K}^{G} \operatorname{Ind}_{H}^{G} q\right)(a g \otimes x) & =\sum_{k \in \mathcal{Q}(K)}\left(\operatorname{Ind}_{H}^{G} q\right)(a g \otimes x)_{k} k \\
& =\sum_{k \in \mathcal{Q}(K)}(a g q(x) \overline{a g})_{k} k
\end{aligned}
$$

and

$$
\begin{aligned}
\left.\operatorname{(Ind}_{K \cap g H g^{-1}}^{K} c_{\left(H \cap g^{-1} K g, g\right)} \# \operatorname{Res}_{H \cap g^{-1} K g}^{H} q\right)(a \otimes(e \otimes x)) & \\
= & \sum_{k \in \mathcal{Q}(K)}\left(a\left(c_{\left(H \cap g^{-1} K g, g\right)} \operatorname{Res}_{H \cap g^{-1} K g}^{H} q\right)(e \otimes x) \bar{a}\right)_{k} k \\
& =\sum_{k \in \mathcal{Q}(K)}\left(a g\left(\operatorname{Res}_{H \cap g^{-1} K g}^{H} q\right)(x) g^{-1} \bar{a}\right)_{k} k \\
& =w(g) \sum_{k \in \mathcal{Q}(K)}(a g q(x) \overline{a g})_{k} k .
\end{aligned}
$$

Proposition 12.7. Let $\boldsymbol{A}_{H}$ and $\Theta_{H}$ be as above for each $H \in \mathcal{S}(G)$. Then the Witt group functor $H \mapsto \mathrm{WQ}_{0}\left(\boldsymbol{A}_{H}, \Theta_{H}\right), H \in \mathcal{S}(G)$, and the special Witt group functor $H \mapsto \operatorname{SWQ}_{0}\left(\boldsymbol{A}_{H}, \Theta_{H}\right), H \in \mathcal{S}(G)$, are both w-Mackey functors, and hence modules over the Burnside ring functor $H \mapsto \Omega(G), H \in \mathcal{S}(G)$.

Proof. The claim for the Witt group functor follows from Propositions 3.2, 3.4, 3.5. 6.6, 6.7, 6.8, 12.4, 12.5, and 12.6

Let $\boldsymbol{M}=(M, B, q, \alpha)$ be a $\Theta_{H}$-positioned $\boldsymbol{A}_{H}$-quadratic $R[H]$-module. By Lemma 6.3, $\varepsilon \circ B: M \times M \rightarrow R$ is a $\lambda$-symmetric, $\left(H,\left.w\right|_{H}\right)$-invariant, $R$-bilinear form. For a morphism $\varphi: H \rightarrow K$ in $\mathcal{G}$, the same argument as the proof of Lemma 11.1 shows that if $\nabla_{\boldsymbol{M}}=0$ (see 8.1) ), then $\nabla_{\varphi_{\#} \boldsymbol{M}}=0$. (In fact, consider the case where $R$ is replaced by $R / 2 R$.) Thus, the claim for the special Witt group functor also follows.

In the remainder of this section, let $\varphi: H \rightarrow K$ be a morphism in $\mathcal{G}$.

Proposition 12.8. Let $\boldsymbol{M}_{1}=\left(M_{1}, B_{1}, \alpha_{1}\right)$ and $\boldsymbol{M}_{2}=\left(M_{2}, B_{2}, q_{2}, \alpha_{2}\right)$ be objects in $\mathcal{H}\left(R, K, \Theta_{K}\right)$ and $\mathcal{Q}\left(\boldsymbol{A}_{H}, \Theta_{H}\right)$, respectively. Let

$$
f: M_{1} \otimes_{R} \varphi_{\#} M_{2} \rightarrow \varphi_{\#}\left(\varphi^{\#} M_{1} \otimes_{R} M_{2}\right)
$$

denote the canonical isomorphism, namely $f\left(x \otimes\left(k \otimes_{\varphi} y\right)\right)=k \otimes_{\varphi}\left(k^{-1} x \otimes y\right)$ for $k \in K, x \in M_{1}$ and $y \in M_{2}$. Then the diagram

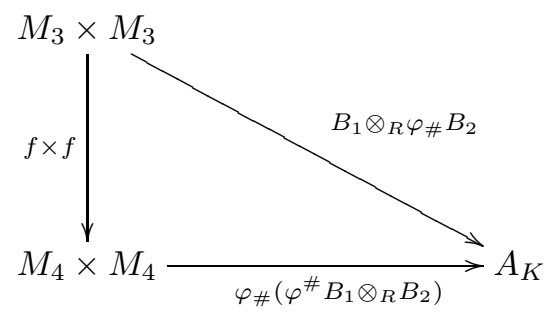


where $M_{3}=\left(M_{1} \otimes_{R}\left(R[K] \otimes_{R[H], \varphi} M_{2}\right)\right)$ and $M_{4}=R[K] \otimes_{R[H], \varphi}\left(\varphi^{\#} M_{1} \otimes_{R} M_{2}\right)$, and the diagram

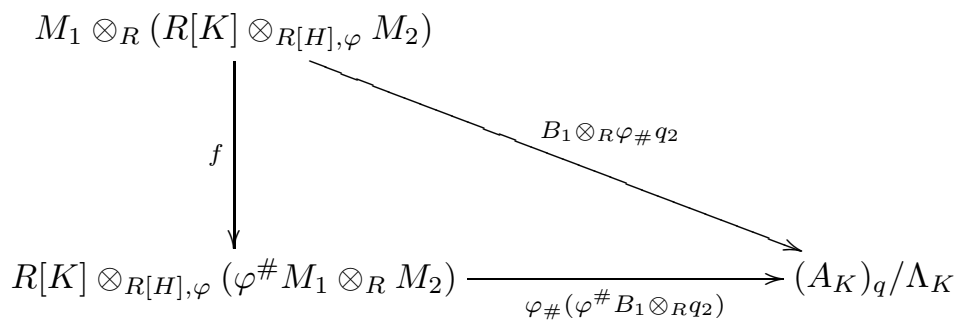

commute.

Proof. Let $k, k^{\prime} \in K, x, x^{\prime} \in M_{1}$, and $y, y^{\prime} \in M_{2}$.

The commutability $B_{1} \otimes\left(\varphi_{\#} B_{2}\right)=\varphi_{\#}\left(\left(\varphi^{\#} B_{1}\right) \otimes B_{2}\right)$ via $f$ holds because

$$
\begin{aligned}
B_{1} \otimes & \left(\varphi_{\#} B_{2}\right)\left(x \otimes\left(k \otimes_{\varphi} y\right), x^{\prime} \otimes\left(k^{\prime} \otimes_{\varphi} y^{\prime}\right)\right) \\
& =\sum_{g \in K} B_{1}\left(x, g^{-1} x^{\prime}\right) \varepsilon\left(\varphi_{\#} B_{2}\left(k \otimes_{\varphi} y, g^{-1}\left(k^{\prime} \otimes_{\varphi} y^{\prime}\right)\right)\right) g \\
& =\sum_{g \in K} w(k) \delta_{k \varphi(H), g^{-1} k^{\prime} \varphi(H)} B_{1}\left(x, g^{-1} x^{\prime}\right) \varepsilon\left(B_{2}\left(y, \varphi^{-1}\left(k^{-1} g^{-1} k^{\prime}\right) y^{\prime}\right)\right) g
\end{aligned}
$$

and

$$
\begin{aligned}
& \varphi_{\#}\left(\left(\varphi^{\#} B_{1}\right) \otimes B_{2}\right)\left(k \otimes_{\varphi}\left(k^{-1} x \otimes y\right), k^{\prime} \otimes_{\varphi}\left(k^{\prime-1} x^{\prime} \otimes y^{\prime}\right)\right) \\
&= \sum_{g \in K} w(k) \delta_{k \varphi(H), g^{-1}} k^{\prime} \varphi(H) \\
& \cdot \varepsilon\left(\left(\varphi^{\#} B_{1} \otimes B_{2}\right)\left(\left(k^{-1} x \otimes y\right), \varphi^{-1}\left(k^{-1} g^{-1} k^{\prime}\right)\left(k^{\prime-1} x^{\prime} \otimes y^{\prime}\right)\right)\right) g \\
&=\sum_{g \in K} w(k) \delta_{k \varphi(H), g^{-1} k^{\prime} \varphi(H)} \\
& \cdot B_{1}\left(k^{-1} x,\left(k^{-1} g^{-1} k^{\prime}\right) k^{\prime-1} x^{\prime}\right) \varepsilon\left(B_{2}\left(y, \varphi^{-1}\left(k^{-1} g^{-1} k^{\prime}\right) y^{\prime}\right)\right) g \\
&=\sum_{g \in K} w(k) \delta_{k \varphi(H), g^{-1} k^{\prime} \varphi(H)} B_{1}\left(k^{-1} x, k^{-1} g^{-1} x^{\prime}\right) \varepsilon\left(B_{2}\left(y, \varphi^{-1}\left(k^{-1} g^{-1} k^{\prime}\right) y^{\prime}\right)\right) g \\
&=\sum_{g \in K} w(k) \delta_{k \varphi(H), g^{-1} k^{\prime} \varphi(H)} B_{1}\left(x, g^{-1} x^{\prime}\right) \varepsilon\left(B_{2}\left(y, \varphi^{-1}\left(k^{-1} g^{-1} k^{\prime}\right) y^{\prime}\right)\right) g
\end{aligned}
$$


The commutability $B_{1} \otimes\left(\varphi_{\#} q_{2}\right)=\varphi_{\#}\left(\left(\varphi^{\#} B_{1}\right) \otimes q_{2}\right)$ via $f$ follows from

$$
\begin{aligned}
B_{1} \otimes\left(\varphi_{\#} q_{2}\right) & \left(x \otimes\left(k \otimes_{\varphi} y\right)\right)=\sum_{g \in \mathcal{Q}(K)} B_{1}\left(x, g^{-1} x\right) \varphi_{\#} q_{2}\left(k \otimes_{\varphi} y\right)_{g} g \\
& =\sum_{g \in \mathcal{Q}(K)} B_{1}\left(x, g^{-1} x\right)\left(k \varphi\left(q_{2}(y)\right) \bar{k}\right)_{g} g \\
& =\sum_{g \in \mathcal{Q}(K)} B_{1}\left(x, g^{-1} x\right) \varphi\left(q_{2}(y)\right)_{k^{-1} g k} w(k) g \\
& =\sum_{g \in \mathcal{Q}(K) \cap k \varphi(H) k^{-1}} B_{1}\left(x, g^{-1} x\right) q_{2}(y)_{\varphi^{-1}\left(k^{-1} g k\right)} w(k) g \\
& =k\left(\sum_{a \in k^{-1} \mathcal{Q}(K) k \cap \varphi(H)} B_{1}\left(x, k a^{-1} k^{-1} x\right) q_{2}(y)_{\varphi^{-1}(a)} a\right) \bar{k} \\
& =k\left(\sum_{b \in \mathcal{Q}(H)} B_{1}\left(x, k \varphi(b)^{-1} k^{-1} x\right) q_{2}(y)_{b} \varphi(b)\right) \bar{k}
\end{aligned}
$$

and

$$
\begin{aligned}
\varphi_{\#}\left(\left(\varphi^{\#} B_{1}\right) \otimes q_{2}\right) & \left.k \otimes_{\varphi}\left(k^{-1} x \otimes y\right)\right)=k \varphi\left(\left(\varphi^{\#} B_{1}\right) \otimes q_{2}\left(k^{-1} x \otimes y\right) \bar{k}\right. \\
& =k \varphi\left(\sum_{h \in \mathcal{Q}(H)} \varphi^{\#} B_{1}\left(k^{-1} x, h^{-1} k^{-1} x\right) q_{2}(y)_{h} h\right) \bar{k} \\
& =k \varphi\left(\sum_{h \in \mathcal{Q}(H)} B_{1}\left(k^{-1} x, \varphi(h)^{-1} k^{-1} x\right) q_{2}(y)_{h} h\right) \bar{k} \\
& =k \varphi\left(\sum_{h \in \mathcal{Q}(H)} B_{1}\left(x, k \varphi(h)^{-1} k^{-1} x\right) q_{2}(y)_{h} h\right) \bar{k} .
\end{aligned}
$$

Proposition 12.9. Let $\boldsymbol{M}_{1}=\left(M_{1}, B_{1}, \alpha_{1}\right)$ and $\boldsymbol{M}_{2}=\left(M_{2}, B_{2}, q_{2}, \alpha_{2}\right)$ be objects in $\mathcal{H}\left(R, H, \Theta_{H}\right)$ and $\mathcal{Q}\left(\boldsymbol{A}_{K}, \Theta_{K}\right)$, respectively. Let

$$
f^{\prime}:\left(\varphi_{\#} M_{1}\right) \otimes_{R} M_{2} \rightarrow \varphi_{\#}\left(M_{1} \otimes_{R} \varphi^{\#} M_{2}\right)
$$

denote the canonical isomorphism, namely $f^{\prime}\left(\left(k \otimes_{\varphi} x\right) \otimes y\right)=k \otimes_{\varphi}\left(x \otimes k^{-1} y\right)$ for $k \in K, x \in M_{1}$ and $y \in M_{2}$. Then the diagram

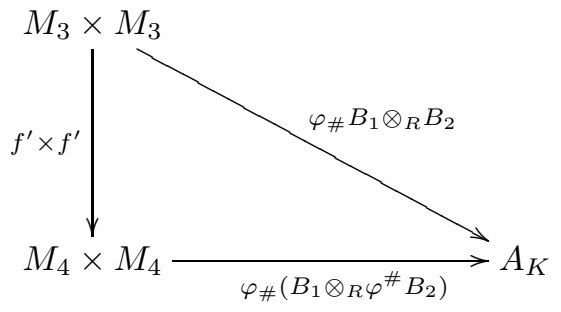


where $M_{3}=\left(R[K] \otimes_{R[H], \varphi} M_{1}\right) \otimes_{R} M_{2}$ and $M_{4}=R[K] \otimes_{R[H], \varphi}\left(M_{1} \otimes_{R} \varphi^{\#} M_{2}\right)$, and the diagram

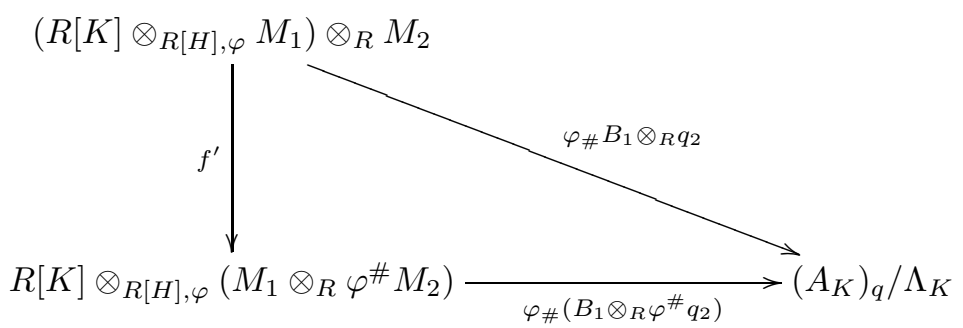

commute.

Proof. Let $k, k^{\prime} \in K, x, x^{\prime} \in M_{1}$, and $y, y^{\prime} \in M_{2}$.

The commutability $\left(\varphi_{\#} B_{1}\right) \otimes B_{2}=\varphi_{\#}\left(B_{1} \otimes\left(\varphi^{\#} B_{2}\right)\right)$ via $f^{\prime}$ holds because $\left(\varphi_{\#} B_{1}\right) \otimes B_{2}\left(\left(k \otimes_{\varphi} x\right) \otimes y,\left(k^{\prime} \otimes_{\varphi} x^{\prime}\right) \otimes y^{\prime}\right)$

$$
\begin{aligned}
& =\sum_{g \in K}\left(\varphi_{\#} B_{1}\right)\left(\left(k \otimes_{\varphi} x\right), g^{-1}\left(k^{\prime} \otimes_{\varphi} x^{\prime}\right)\right) \varepsilon\left(B_{2}\left(y, g^{-1} y^{\prime}\right)\right) g \\
& =\sum_{g \in K} \delta_{k \varphi(H), g^{-1} k^{\prime} \varphi(H)} B_{1}\left(x, \varphi^{-1}\left(k^{-1} g^{-1} k^{\prime}\right) x^{\prime}\right) \varepsilon\left(B_{2}\left(y, g^{-1} y^{\prime}\right)\right) g
\end{aligned}
$$

and

$$
\begin{aligned}
& \varphi_{\#}\left(B_{1} \otimes\left(\varphi^{\#} B_{2}\right)\right)\left(k \otimes_{\varphi}\left(x \otimes k^{-1} y\right), k^{\prime} \otimes_{\varphi}\left(x^{\prime} \otimes k^{\prime-1} y^{\prime}\right)\right) \\
&= \sum_{g \in K} w(k) \delta_{k \varphi(H), g^{-1} k^{\prime} \varphi(H)} \\
& \cdot \varepsilon\left(B_{1} \otimes\left(\varphi^{\#} B_{2}\right)\left(x \otimes k^{-1} y, \varphi^{-1}\left(k^{-1} g^{-1} k^{\prime}\right)\left(x^{\prime} \otimes k^{\prime-1} y^{\prime}\right)\right)\right) g \\
&=\sum_{g \in K} w(k) \delta_{k \varphi(H), g^{-1} k^{\prime} \varphi(H)} B_{1}\left(x, \varphi^{-1}\left(k^{-1} g^{-1} k^{\prime}\right) x^{\prime}\right) \varepsilon\left(B_{2}\left(k^{-1} y, k^{-1} g^{-1} y^{\prime}\right)\right) g \\
&=\sum_{g \in K} \delta_{k \varphi(H), g^{-1} k^{\prime} \varphi(H)} B_{1}\left(x, \varphi^{-1}\left(k^{-1} g^{-1} k^{\prime}\right) x^{\prime}\right) \varepsilon\left(B_{2}\left(y, g^{-1} y^{\prime}\right)\right) g .
\end{aligned}
$$

The commutability $\left(\varphi_{\#} B_{1}\right) \otimes q_{2}=\varphi_{\#}\left(B_{1} \otimes\left(\varphi^{\#} q_{2}\right)\right)$ via $f^{\prime}$ follows from

$$
\begin{aligned}
\left(\varphi_{\#} B_{1}\right) \otimes q_{2} & \left(\left(k \otimes_{\varphi} x\right) \otimes y\right)=\sum_{g \in \mathcal{Q}(K)}\left(\varphi_{\#} B_{1}\right)\left(k \otimes_{\varphi} x, g^{-1}\left(k \otimes_{\varphi} x\right)\right) q_{2}(y)_{g} g \\
& =\sum_{g \in \mathcal{Q}(K)} \delta_{k \varphi(H), g^{-1}} k \varphi(H) B_{1}\left(x, \varphi^{-1}\left(k^{-1} g^{-1} k\right) x\right) q_{2}(y)_{g} g \\
& =\sum_{g \in \mathcal{Q}\left(k \varphi(H) k^{-1}\right)} B_{1}\left(x, \varphi^{-1}\left(k^{-1} g^{-1} k\right) x\right) q_{2}(y)_{g} g \\
& =k \sum_{h \in \mathcal{Q}(H)} B_{1}\left(x, h^{-1} x\right) q_{2}(y)_{k \varphi(h) k^{-1}} \varphi(h) k^{-1} \\
& =k \sum_{h \in \mathcal{Q}(H)} w(k) B_{1}\left(x, h^{-1} x\right) q_{2}\left(k^{-1} y\right)_{\varphi(h)} \varphi(h) k^{-1} \\
& =k \varphi\left(\sum_{h \in \mathcal{Q}(H)} B_{1}\left(x, h^{-1} x\right) q_{2}\left(k^{-1} y\right)_{\varphi(h)} h\right) \bar{k}
\end{aligned}
$$


and

$$
\begin{aligned}
\varphi_{\#}\left(B_{1} \otimes\left(\varphi^{\#} q_{2}\right)\right)\left(k \otimes_{\varphi}\left(x \otimes k^{-1} y\right)\right) & =k \varphi\left(B_{1} \otimes\left(\varphi^{\#} q_{2}\right)\left(x \otimes k^{-1} y\right)\right) \bar{k} \\
& =k \varphi\left(\sum_{h \in \mathcal{Q}(H)} B_{1}\left(x, h^{-1} x\right) q_{2}\left(k^{-1} y\right)_{\varphi(h)} h\right) \bar{k}
\end{aligned}
$$

Let $\psi: \Theta_{H} \rightarrow \Theta_{K}$ denote the map associated with $\varphi$.

Theorem 12.10. Let $A_{H}$ and $\Theta_{H}$ be as above for each $H \in \mathcal{S}(G)$. Then the $w$-Mackey functor $H \mapsto \mathrm{WQ}_{0}\left(\boldsymbol{A}_{H}, \Theta_{H}\right), H \in \mathcal{S}(G)$, is a module over the Green functor $H \mapsto \mathrm{GW}_{0}\left(R, H, \Theta_{H}\right), H \in \mathcal{S}(G)$. If $R$ is square identical, then the $w$ Mackey functor $H \mapsto \mathrm{SWQ}_{0}\left(\boldsymbol{A}_{H}, \Theta_{H}\right), H \in \mathcal{S}(G)$, is a module over the Green functor $H \mapsto \operatorname{SGW}_{0}\left(R, H, S_{H}, \Theta_{H}\right), H \in \mathcal{S}(G)$.

Proof. By Proposition 3.3 we have $\alpha_{1} \otimes\left(\psi_{\#} \alpha_{2}\right)=\psi_{\#}\left(\left(\psi^{\#} \alpha_{1}\right) \otimes \alpha_{2}\right)$ via $f$ in Proposition 12.8 By Proposition 3.3 we have $\left(\psi_{\#} \alpha_{1}\right) \otimes \alpha_{2}=\psi_{\#}\left(\alpha_{1} \otimes \psi^{\#} \alpha_{2}\right)$ via $f^{\prime}$ in Proposition 12.9. The theorem follows from Propositions 12.2 12.8 and 12.9.

\section{Applications of induCtion and Restriction}

Let $Z^{0}$ be a finite $G$-set, and let $\mathcal{S}(G) \rightarrow \mathfrak{P}\left(Z^{0}\right) ; H \mapsto Z_{H}^{(0)}$ be an intersectionpreserving $G$-map. Let $S$ be a conjugation-invariant subset of $G(2)$. We set $S_{H}=$ $H \cap S$ and $\Theta_{H}=S_{H} \amalg Z_{H}^{(0)}$. Define $\rho_{H}^{(2)}: \Theta_{H} \rightarrow \mathfrak{P}\left(S_{H}\right)$ by

$$
\rho_{H}^{(2)}(t)= \begin{cases}\{t\} & \left(t \in S_{H}\right), \\ \emptyset & \left(t \in Z_{H}^{(0)}\right) .\end{cases}
$$

Further, let $\mathcal{F}$ be a conjugation-invariant subset of $\mathcal{S}(G)$ such that

$$
\Theta_{G} \times \Theta_{G}=\bigcup_{H \in \mathcal{F}} \Theta_{H} \times \Theta_{H}
$$

and let $\beta$ be an element in the Burnside ring $\Omega(G)$ such that

$$
\operatorname{Res}_{H}^{G} \beta=1_{\Omega(H)} \quad \text { for any } H \in \mathcal{F} \text {. }
$$

Theorem 13.1. Let $x$ be an arbitrary element in $\operatorname{SGW}_{0}\left(R, G, S, \Theta_{G}\right)$. If $\mathcal{F}$ contains all 2-hyperelementary (resp. cyclic) subgroups of $G$, then $\left(1_{\Omega(G)}-\beta\right)^{2} x=0$ (resp. $\left(1_{\Omega(G)}-\beta\right)^{2 k+3} x=0$, where $k$ is the integer such that $|G|=2^{k} m$ with an odd integer $m)$.

For the proof, we recall two lemmas.

Lemma 13.2 (A. Dress [11] Theorems 1 and 3]). For a set $\mathcal{H}$ of subgroups of $G$, the restriction homomorphism

$$
\text { Res : } \mathrm{GW}_{0}(\mathbb{Z}, G) \rightarrow \bigoplus_{H \in \mathcal{H}} \mathrm{GW}_{0}(\mathbb{Z}, H)
$$

has the following properties.

(1) If $\mathcal{H}$ contains all 2-hyperelementary subgroups of $G$, then Res is injective.

(2) If $\mathcal{H}$ contains all cyclic subgroups of $G$, then the kernel of Res is annihilated by 4 . 
For a subgroup $H$ of $G$, we denote by $\chi_{H}$ the homomorphism $\Omega(G) \rightarrow \mathbb{Z}$ such that $\chi_{H}([X])=\left|X^{H}\right|$ for every finite $G$-set $X$.

Lemma 13.3 ([15, Proposition 6.3]). Let $x$ be an element of $\Omega(G)$ such that $\chi_{H}(x) \equiv 0 \bmod 2$ for all $H \in \mathcal{S}(G)$. Then $x^{k+1}$ lies in $2 \Omega(G)$, where $k$ is the integer such that $|G|=2^{k} m$ with an odd integer $m$.

Proof of Theorem 13.1. Let $H$ be a 2-hyperelementary subgroup of $G$.

First consider the case where $\mathcal{F}$ contains all 2-hyperelementary subgroups of $G$. Then, it is obvious that $\operatorname{Res}_{H}^{G}\left(1_{\Omega(G)}-\beta\right)=0$. Since the Green functor $\mathrm{GW}_{0}(\mathbb{Z},-)$ is a module over the Green functor $\Omega(-), \operatorname{Res}_{H}^{G}\left(\left(1_{\Omega(G)}-\beta\right) \mathrm{GW}_{0}(\mathbb{Z}, G)\right)=0$.

Next, consider the case where $\mathcal{F}$ contains all cyclic subgroups of $G$. Then

$$
\chi_{K}\left(1_{\Omega(G)}-\beta\right) \equiv 0 \quad \bmod 2
$$

for any subgroup $K$ of $H$, and hence $\operatorname{Res}_{H}^{G}\left(1_{\Omega(G)}-\beta\right)^{2 k+2}$ lies in $4 \Omega(H)$. So, we can write $\operatorname{Res}_{H}^{G}\left(1_{\Omega(G)}-\beta\right)^{2 k+2}=4 \gamma$ for some $\gamma \in \Omega(H)$. Clearly, $\operatorname{Res}_{C}^{H} \gamma=0$ for all cyclic subgroups of $H$. Thus by (2) of Dress' Lemma, $\gamma \mathrm{GW}_{0}(\mathbb{Z}, H)$ is annihilated by 4 , and hence $\operatorname{Res}_{H}^{G}\left(\left(1_{\Omega(G)}-\beta\right)^{2 k+2} \mathrm{GW}_{0}(\mathbb{Z}, G)\right)=0$. By (1) of Dress' Lemma, we obtain

$$
(1-\beta) \mathrm{GW}_{0}(\mathbb{Z}, G)=0 \text { or }\left(1_{\Omega(G)}-\beta\right)^{2 k+2} \mathrm{GW}_{0}(\mathbb{Z}, G)=0 .
$$

Since the canonical map $\mathrm{GW}_{0}(\mathbb{Z}, G) \rightarrow \mathrm{GW}_{0}(R, G)$ is an $\Omega(G)$-homomorphism of a ring with unit, it follows that

$$
(1-\beta) \mathrm{GW}_{0}(R, G)=0 \text { or }\left(1_{\Omega(G)}-\beta\right)^{2 k+2} \mathrm{GW}_{0}(R, G)=0 .
$$

Noting that the Mackey functor $\operatorname{SGW}_{0}\left(R,-, S_{-}\right)$is a module over the Green functor $\mathrm{GW}_{0}(R,-)$, we obtain

$$
(1-\beta) \mathrm{SGW}_{0}(R, G, S)=0 \text { or }\left(1_{\Omega(G)}-\beta\right)^{2 k+2} \mathrm{SGW}_{0}(R, G, S)=0 .
$$

Recall Proposition 5.3. namely the fact that the canonical homomorphism

$$
\mathrm{SGW}_{0}(R, G, S) \rightarrow \mathrm{SGW}_{0}\left(R, G, S, \Theta_{G}\right)^{\mathrm{t} \text {-iso }}
$$

is surjective. In addition, the homomorphism is an $\Omega(G)$-homomorphism. Hence, we conclude that

$(1-\beta) \mathrm{SGW}_{0}\left(R, G, S, \Theta_{G}\right)^{\mathrm{t} \text {-iso }}=0$ or $\left(1_{\Omega(G)}-\beta\right)^{2 k+2} \mathrm{SGW}_{0}\left(R, G, S, \Theta_{G}\right)^{\mathrm{t} \text {-iso }}=0$.

On the other hand, it is easy to check that $(1-\beta) \operatorname{SGW}_{0}\left(R, G, S, \Theta_{G}\right)$ is contained in (the image by the canonical map from) $\operatorname{SGW}_{0}\left(R, G, S, \Theta_{G}\right)^{\mathrm{t} \text {-iso }}$.

Putting all together, we establish that

$$
(1-\beta)^{2} \mathrm{SGW}_{0}\left(R, G, S, \Theta_{G}\right)=0 \text { or }\left(1_{\Omega(G)}-\beta\right)^{2 k+3} \mathrm{SGW}_{0}\left(R, G, S, \Theta_{G}\right)=0 .
$$

Proof of Theorem 1.2. Here $Z^{(0)}$ is the empty set. Since $H \mapsto \operatorname{SGW}\left(R, H, S_{H}, S_{H}\right)$ is a Mackey functor, it is a module over the Burnside ring functor $H \mapsto \Omega(H)$ by 7, Proposition 6.2.3]. For each subgroup $H$ of $G$ we have

$$
\Theta_{H} \times \Theta_{H}=(S \cap H) \times(S \cap H)=(S \times S) \cap(H \times H) .
$$

Thus (13.1) is fulfilled, and Theorem 1.2 follows from Theorem 13.1 
Now let $w: G \rightarrow\{-1,1\}$ be a homomorphism, $\lambda=1$ or -1 , and let $Q$ be a conjugation-invariant subset of $G^{-\lambda}(2)$. Suppose $S \subset G^{\lambda}(2)$. For each $H \in \mathcal{S}(G)$, we set $A_{H}=R[H], Q_{H}=H \cap Q$, and $\boldsymbol{A}_{H}=\left(R, H, Q_{H}, S_{H}, \lambda,\left.w\right|_{H}\right)$.

Theorem 13.4. Suppose $R$ is square identical. Let $x$ be an arbitrary element of the special Witt group $\operatorname{SWQ}_{0}\left(\boldsymbol{A}_{G}, \Theta_{G}\right)$. If $\mathcal{F}$ contains all 2-hyperelementary (resp. cyclic) subgroups of $G$, then $\left(1_{\Omega(G)}-\beta\right)^{2} x=0$ (resp. $\left(1_{\Omega(G)}-\beta\right)^{2 k+3} x=0$, where $|G|=2^{k} m$ with $m$ odd).

Proof. The theorem follows from Proposition 12.10 and Theorem 13.1.

Proof of Theorem 1.3. Theorem 1.3 follows from Theorem 13.4.

Theorem 13.5. Suppose that $R$ is square identical, $\mathcal{F}$ contains any cyclic subgroup of $G$, and $\beta$ has the form

$$
\beta=\sum_{H \in \widetilde{\mathcal{F}}} n_{H}[G / H]
$$

with $n_{H} \in \mathbb{Z}$ for some lower closed subset $\widetilde{\mathcal{F}}$ of $\mathcal{S}(G)$; namely, any subgroup $H$ of $G$ lies in $\widetilde{\mathcal{F}}$ whenever $K \in \widetilde{\mathcal{F}}$ and $H \subset K$. Then

$$
\operatorname{SWQ}_{0}\left(R, G, Q, S, \Theta_{G}\right)=\sum_{H \in \widetilde{\mathcal{F}}} \operatorname{Ind}_{H}^{G} \operatorname{SWQ}_{0}\left(R, H, Q_{H}, S_{H}, \Theta_{H}\right),
$$

and the restriction homomorphism

$$
\text { Res : } \operatorname{SWQ}_{0}\left(R, G, Q, S, \Theta_{G}\right) \rightarrow \bigoplus_{H \in \widetilde{\mathcal{F}}} \operatorname{SWQ}_{0}\left(R, H, Q_{H}, S_{H}, \Theta_{H}\right)
$$

is injective.

Proof. By hypothesis, we can write

$$
\left(1_{\Omega(G)}-\beta\right)^{2|G|+3}=[G / G]-\sum_{H \in \widetilde{\mathcal{F}}} m_{H}[G / H]
$$

with $m_{H} \in \mathbb{Z}$. For an arbitrary element $x \in \mathrm{SWQ}_{0}\left(R, G, Q, S, \Theta_{G}\right)$, Theorem 13.4 implies that

$$
x=\sum_{H \in \widetilde{\mathcal{F}}} m_{H}[G / H] \cdot x=\sum_{H \in \widetilde{\mathcal{F}}} m_{H} \operatorname{Ind}_{H}^{G}\left(\operatorname{Res}_{H}^{G} x\right) .
$$

Moreover, if $\operatorname{Res}_{H}^{G} x=0$ for every $H \in \widetilde{\mathcal{F}}$, then we conclude that $x=0$.

Proof of Theorem 1.4 Since $G$ is a nonsolvable group, there exists an idempotent $\beta \in \Omega(G)$ such that $\chi_{K}(\beta)=0$ for any nonsolvable subgroup $K$ of $G$ and $\chi_{H}(\beta)=1$ for any solvable subgroup $H$ of $G$. This element $\beta$ has the form $\beta=\sum_{H} n_{H}[G / H]$ with $n_{H} \in \mathbb{Z}$, where $H$ runs over the set of all solvable subgroups of $G$. Thus, Theorem 1.4 follows from Theorem 13.5 .

\section{REFERENCES}

1. A. Bak, K-Theory of Forms, Annals of Mathematics Studies 98, Princeton Univ. Press, Princeton, 1981. MR 84m:10012

2. A. Bak, Induction for finite groups revisited, J. Pure and Applied Algbera 104 (1995), pp. 235241. MR 96m:19002 
3. A. Bak and M. Morimoto, Equivariant surgery and applications, in: Proceedings of Conference on Topology in Hawaii 1990 (ed. K. H. Dovermann), pp. 13-25, World Scientific Publ., Singapore, 1992. MR 93e:57058

4. A. Bak and M. Morimoto, K-theoretic groups with positioning map and equivariant surgery, Proc. Japan Acad. 70 Ser. A (1994), 6-11. MR 95e:19006

5. A. Bak and M. Morimoto, Equivariant surgery with middle-dimensional singular sets. I, Forum Math. 8 (1996), 267-302. MR 97b:57031

6. A. Bak and W. Scharlau, Grothendieck and Witt groups of orders and finite groups, Invent. Math. 23 (1974), 207-240. MR 49:5093

7. T. tom Dieck, Transformation Groups and Representation Theory, Lecture Notes in Mathematics 766, Springer-Verlag, Berlin-Heidlberg-New York, 1979. MR 82c:57025

8. T. tom Dieck, Transformation Groups, de Gruyter Studies in Mathematics 8, Walter de Gruyter, Berlin, 1987. MR 89c:57048

9. A. Dress, A characterization of solvable groups, Math. Zeit. 110 (1969), 213-217. MR 40:1491

10. A. Dress, Contributions to the theory of induced representations, in: Algebraic $K$-theory, II: "Classical" algebraic $K$-theory and connections with arithmetic, Proc. Conf., Battelle Memorial Inst., Seattle, 1972, Lecture Notes in Mathematics 342, pp. 183-240, SpringerVerlag, Berlin-Heidlberg-New York, 1973. MR 52:5787

11. A. Dress, Induction and structure theorems for Grothendieck and Witt rings of orthogonal representations of finite groups, Bull. Amer. Math. Soc. 79 (1973), 741-745. MR 49:7345

12. A. Dress, Induction and structure theorems for orthogonal representations of finite groups, Ann. of Math. 102 (1975), 291-325. MR 52:8235

13. I. Hambleton and L. Taylor, A guide to the calculation of the surgery obstruction groups for finite groups, in: Surveys on Surgery Theory, vol. 1 (ed. S. Cappell, A. Ranicki and J. Rosenberg), Annals of Mathematics Studies 145, pp. 225-274, Princeton Univ. Press, Princeton, 2000. MR 2001e:19007

14. I. Hambleton, L. Taylor and B. Williams, An introduction to maps between surgery obstruction groups, in: Algebraic Topology, Aarhus 1982, Lecture Notes in Mathematics 1051, pp. 29-127, Springer-Verlag, Berlin-Heidelberg-New York, 1984. MR 86b:57017

15. E. Laitinen and M. Morimoto, Finite groups with smooth one fixed point actions on spheres, Forum Math. 10 (1998), 479-520. MR 99k:57078

16. E. Laitinen, M. Morimoto and K. Pawałowski, Deleting-inserting theorem for smooth actions of finite solvable groups on spheres, Comment. Math. Helvetica 70 (1995), 10-38. MR 96b:57043

17. M. Morimoto, On one fixed point actions on spheres, Proc. Japan Acad. 63 Ser. A Math. Sci. (1987), 95-97. MR 88j:57039

18. M. Morimoto, Most of the standard spheres have one fixed point actions of $A_{5}$, in: Transformation Groups (ed. K. Kawakubo), Lecture Notes in Mathematics 1375, pp. 240-258, Springer-Verlag, Berlin-Heidlberg-New York, 1989. MR 90i:57029

19. M. Morimoto, Bak groups and equivariant surgery, K-Theory 2 (1989), 465-483. MR 90d:57037

20. M. Morimoto, Most standard spheres have smooth one fixed point actions of $A_{5}$. II, K-Theory 4 (1991), 289-302. MR 92h:57055

21. M. Morimoto, Equivariant surgery theory: Deleting-inserting theorems of fixed point manifolds on spheres and disks, $K$-Theory 15 (1998), 13-32. MR 99i:57056

22. M. Morimoto, Equivariant surgery with middle dimensional singular sets. II: Equivariant framed cobordism invariance, Trans. Amer. Math. Soc. 353 (2001), 2427-2440. MR 2001m:57060

23. M. Morimoto, The Burnside ring revisited, in: Current Trends in Transformation Groups (eds. A. Bak, M. Morimoto and F. Ushitaki), K-Monographs in Mathematics 7, pp. 129-145, Kluwer Academic Publishers, Dordrecht-Boston-London, 2002.

24. M. Morimoto and K. Pawałowski, Smooth actions of finite Oliver groups on spheres, Topology 42 (2003), 395-421.

25. M. Morimoto and K. Uno, Remarks on one fixed point $A_{5}$-actions on homotopy spheres, in: Algebraic Topology Poznań 1989 (eds. S. Jackowski, B. Oliver and K. Pawałowski), Lecture Notes in Mathematics 1478, pp. 337-364, Springer-Verlag, Berlin-Heidlberg-New York, 1991. MR 92i:57031 
26. T. Petrie, One fixed point actions on spheres I, Advances in Mathematics 46 (1982), 3-14. MR 84b:57027

27. C. T. C. Wall, Surgery on Compact Manifolds, London Mathematical Society Monographs, No. 1, Academic Press, London-New York, 1970. MR 55:4217

Department of Environmental and Mathematical Sciences, Faculty of Environmental Science and Technology, Okayama University, Okayama, 700-8530 Japan

E-mail address: morimoto@ems.okayama-u.ac.jp 